CLNS 91/1120

September 1992

\title{
GENERALIZED GLUON CURRENTS AND APPLICATIONS IN QCD
}

\author{
Gregory MAHLON ${ }^{1}$ and Tung-Mow YAN ${ }^{2}$ \\ Newman Laboratory of Nuclear Studies, \\ Cornell University, Ithaca, NY 14853, USA \\ Charles DUNN ${ }^{3}$ \\ Jet Propulsion Laboratory, M/S 238-600, \\ 4800 Oak Grove Drive, \\ Pasadena, CA 91109, USA
}

\begin{abstract}
We consider the process containing two quark lines and an arbitrary number of gluons in a spinor helicity framework. A current with two off-shell gluons appears in the amplitude. We first study this modified gluon current using recursion relations. The recursion relation for the modified gluon current is solved for the case of like-helicity gluons. We apply the modified gluon current to compute the amplitude for $q \bar{q} \rightarrow q \bar{q} g g \cdot \cdots g$ in the like-helicity gluon case.
\end{abstract}

1 e-mail: gdm@beauty.tn.cornell.edu

2 e-mail: yan@Inssun5.tn.cornell.edu

3 e-mail: ced@logos.jpl.nasa.gov 


\section{INTRODUCTION}

In recent years, there has been a great interest in studying the high energy scatterings in QCD. An efficient approach in the tree approximation is to formulate recursion relations for currents with $n$ gluons in terms of those with fewer gluons [1]. The gluon currents which have been studied so far contain only one off-shell gluon. These are appropriate for processes involving only gluons, or a single quark line plus gluons. However, as soon as we consider processes with two or more quark lines, we encounter gluon currents with two or more off-shell gluons. These currents also satisfy recursion relations similar to those for currents with one off-shell gluon.

In this paper we will study the process

$$
q \bar{q} \longrightarrow q \bar{q} g g \cdots g
$$

Because of the non-Abelian nature of QCD, the number of Feynman diagrams for this process is enormous, even at tree-level. This is true even in cases that are far simpler than the one being studied here. Thus, a suitable means to organize and simplify the calculation is required. A fruitful concept in this respect has been the multispinor representation of a vector field [2]. Over the last 10 years or so, a whole industry devoted to the application of the spinor technique to multi-parton processes has sprung up, employing the spinors in a number of different contexts $[3,4]$. An excellent guide to the many approaches and methods developed for such calculations is the review by Mangano and Parke [5]. We will organize our calculation within the framework of the recursion relations presented in reference [1]. The multispinor representation allows us to treat quarks 
and gluons on an equal footing, while the recursion relations supply connections between currents with $n$ particles and those with $n-1$ and $n-2$ particles. Once we have the recursion relations, we can use their solutions as the starting point for our calculations. Rather than a large number of Feynman diagrams showing all of the possible gluon configurations, we consult a handful of diagrams built from the individual currents. We will use Weyl-van der Waerden spinors in this work (a summary of our conventions appears in the appendix).

The organization of this paper is as follows. In section 2 , we briefly review the recursion relations for quarks and gluons involving only one off-shell particle. We also recount the solutions to these relations for the case in which all of the gluons have the same helicity. In section 3, we study the gluon recursion relation for a gluon current with $n$ off-shell gluons plus any number of on-shell gluons. We then specialize to the case $n=2$ and introduce a "modified" current. This modified current will have a "special" gluon, which is off mass-shell and has a modified "polarization spinor." This recursion relation for the modified current is easily solved when the modified "polarization spinor" for the special gluon assumes a certain form, and the gluons have like helicity. We present a closed form solution for the modified gluon current for this special case. In section 4, we show how the modified gluon current fits into a computation of the process (1.1). We then do the actual calculation for (1.1) for the case in which all of the gluons have the same helicity. Mangano [6] has obtained an expression for this amplitude by using the fact that each color configuration corresponds to a gauge invariant amplitude. Requiring the correct collinear and soft limits in the amplitude leads to the form published in reference [6]. We find that we are able to reproduce Mangano's result using an appropriate combination of quark and gluon currents as the starting point. Hopefully, the techniques and details 
presented here will provide a stimulus for the development of new methods to tackle even more difficult problems. The final section contains a few concluding remarks.

\section{THE QCD RECURSION RELATIONS AND THEIR SOLUTIONS}

In this section we will review the recursion relations for quarks and gluons and their closed form solutions for special helicity configurations presented in reference [1]. Each of the currents discussed below has only one off-shell particle.

\subsection{The multi-gluon current}

We define the current $\widehat{J}_{\xi}^{x}(1, \ldots, n)$ for one off-shell plus $n$ on-shell transverse gauge bosons in the tree approximation. By convention, all momenta will flow into the graph. The $j$ th gauge boson will have momentum $k_{j}$, with the off-shell boson having momentum $k_{n+1}=-\left(k_{1}+k_{2}+\cdots+k_{n}\right) \equiv-\kappa(1, n)$. The color index of the off-shell boson is $x$, while its Lorentz index is $\xi$. We include the propagator for the off-shell boson in the definition of $\widehat{J}_{\xi}^{x}(1, \ldots, n)$. This definition gives

$$
\begin{aligned}
& \widehat{J}_{\xi}^{x}(1, \ldots, n)= \\
& =\frac{i}{\kappa^{2}(1, n)} \sum_{\mathcal{P}(1 \ldots n)}\left\{g \sum_{j=1}^{n-1} \frac{1}{2 ! j !(n-j) !} f^{x_{1} x_{2} x}\right. \\
& \quad \times V^{\mu \nu}(\kappa(1, j), \kappa(j+1, n),-\kappa(1, n)) \\
& \quad \times \widehat{J}_{\mu}^{x_{1}}(1, \ldots, j) \widehat{J}_{\nu}^{x_{2}}(j+1, \ldots, n) \\
& +i g^{2} \sum_{j=1}^{n-2} \sum_{\ell=j+1}^{n-1} \frac{1}{3 ! j !(j-\ell) !(n-\ell) !} \sum_{\mathcal{C}(123)} f^{a_{1} a_{2} y} f^{y a_{3} x} \\
& \left.\times K^{\alpha_{1} \alpha_{2} \alpha_{3}} \widehat{F}_{\xi}^{a_{\alpha_{1}}}(1, \ldots, j) \widehat{J}_{\alpha_{2}}^{a_{2}}(j+1, \ldots, \ell) \widehat{J}_{\alpha_{3}}^{a_{3}}(\ell+1, \ldots, n)\right\}
\end{aligned}
$$


In (2.1) $f^{a b c}$ is the structure constant of the group $S U(N)$. We denote the fundamental matrix representation of $S U(N)$ by $T^{a}$, hence,

$$
\left[T^{a}, T^{b}\right]=i f^{a b c} T^{c}
$$

The T's are normalized such that

$$
\operatorname{tr} T^{a} T^{b}=\frac{1}{2} \delta^{a b}
$$

and satisfy the completeness relation

$$
\sum_{x=1}^{N^{2}-1}\left(T^{x}\right)_{i j}\left(T^{x}\right)_{k \ell}=\frac{1}{2}\left[\delta_{j k} \delta_{i \ell}-\frac{1}{N} \delta_{i j} \delta_{k \ell}\right] .
$$

The functions $V_{\mu \nu \xi}\left(k_{1}, k_{2}, k_{3}\right)$ and $K_{\alpha_{1} \alpha_{2} \alpha_{3} \alpha_{4}}$ represent the 3-vertex and 4-vertex respectively:

$$
\begin{gathered}
V_{\mu \nu \xi}\left(k_{1}, k_{2}, k_{3}\right)=g_{\mu \nu}\left(k_{1}-k_{2}\right)_{\xi}+g_{\nu \xi}\left(k_{2}-k_{3}\right)_{\mu}+g_{\xi \mu}\left(k_{3}-k_{1}\right)_{\nu} \\
K_{\kappa \lambda \mu \nu}=g_{\kappa \nu} g_{\lambda \mu}-g_{\kappa \mu} g_{\lambda \nu} .
\end{gathered}
$$

The symbol $\mathcal{P}(1 \ldots n)$ denotes permutations among $\{1, \ldots, n\}$, while $\mathcal{C}(1 \ldots n)$ represents cyclic permutations.

Berends and Giele [1] show that $\widehat{J}_{\xi}^{x}(1, \ldots, n)$ satisfies the following factorization property and recursion relation:

$$
\begin{gathered}
\widehat{J}_{\xi}^{x}(1, \ldots, n)=2 g^{n-1} \sum_{\mathcal{P}(1 \ldots n)} \operatorname{tr}\left(\Omega[1, n] T^{x}\right) J_{\xi}(1, \ldots, n), \\
J_{\xi}(1, \ldots, n)=\frac{1}{\kappa^{2}(1, n)}\left(\sum_{j=1}^{n-1}[J(1, \ldots, j), J(j+1, \ldots, n)]_{\xi}\right. \\
\left.+\sum_{j=1}^{n-2} \sum_{\ell=j+1}^{n-1}\{J(1, \ldots, j), J(j+1, \ldots, \ell), J(\ell+1, \ldots, n)\}_{\xi}\right), \\
J(1)=\epsilon(1) .
\end{gathered}
$$


In (2.7) we have the notations:

$$
\begin{gathered}
\Omega[1, n] \equiv T^{a_{1}} \ldots T^{a_{n}}, \\
{[J(1, \ldots, j), J(j+1, \ldots, n)]_{\xi}=} \\
=2 \kappa(j+1, n) \cdot J(1, \ldots, j) J_{\xi}(j+1, \ldots, n) \\
-2 \kappa(1, j) \cdot J(j+1, \ldots, n) J_{\xi}(1, \ldots, j) \\
+J(1, \ldots, j) \cdot J(j+1, \ldots, n)[\kappa(1, j)-\kappa(j+1, n)]_{\xi},
\end{gathered}
$$

and

$$
\begin{aligned}
\{J(1), J(2), J(3)\}_{\xi}= & J(1) \cdot\left[J(3) J_{\xi}(2)-J(2) J_{\xi}(3)\right] \\
& -J(3) \cdot\left[J(2) J_{\xi}(1)-J(1) J_{\xi}(2)\right] .
\end{aligned}
$$

The current $J(1, \ldots, n)$ satisfies the following properties [1]:

$$
\begin{gathered}
J(1,2, \ldots, n)=(-1)^{n-1} J(n, n-1, \ldots, 1), \\
\sum_{\mathcal{C}(1 \ldots n)} J(1, \ldots, n)=0 \\
\sum_{j=1}^{n} J(2, \ldots, j, 1, j+1, \ldots, n)=0 \\
\kappa(1, n) \cdot J(1, \ldots, n)=0 .
\end{gathered}
$$

The recursion relation (2.7) may be solved easily for any number of gauge bosons in two special helicity configurations. The key ingredient [1] is the ability to choose gauge spinors such that

$$
\epsilon_{\alpha \dot{\alpha}}(i) \dot{\epsilon}^{\dot{\alpha} \alpha}(j)=0
$$

for any pair of gauge bosons $i$ and $j$. Thus, to consider the situation in which all of the gauge bosons have the same helicity (positive, for concreteness), we choose

$$
\epsilon_{\alpha \dot{\alpha}}\left(j^{+}\right)=\frac{u_{\alpha}(h) \bar{u}_{\dot{\alpha}}\left(k_{j}\right)}{\langle j h\rangle}, \quad j=1,2, \ldots, n
$$


with the same arbitrary null-momentum $h$ for each particle. We will not have occasion to utilize the solution for the situation in which one of the gauge bosons has negative helicity: hence, we will not present it here. The interested reader is referred to reference [1].

The gauge choice (2.13) has the following consequences for the currents:

$$
\begin{gathered}
J(1, \ldots, j) \cdot J(\ell, \ldots, n)=0, \\
\{J(1, \ldots, j), J(j+1, \ldots, \ell), J(\ell+1, \ldots, n)\}=0,
\end{gathered}
$$

for any values of $j, \ell$, and $n$. Furthermore, the square bracket function simplifies to

$$
\begin{aligned}
{[J(1, \ldots, j), J(j+1, \ldots, n)]_{\xi}=} & \\
= & 2 \kappa(j+1, n) \cdot J(1, \ldots, j) J_{\xi}(j+1, \ldots, n) \\
& \quad-2 \kappa(1, j) \cdot J(j+1, \ldots, n) J_{\xi}(1, \ldots, j) .
\end{aligned}
$$

We may use these simplifications plus some help from the Schouten identity (A.8) to write the recursion relation (2.7) in spinor form as

$$
\mathcal{J}_{\alpha \dot{\alpha}}(1, \ldots, n)=\frac{-\sqrt{2}}{\kappa^{2}(1, n)} \sum_{j=1}^{n-1} \mathcal{J}_{\alpha \dot{\beta}}(1, \ldots, j) \overline{\mathcal{J}}^{\dot{\beta} \beta}(j+1, \ldots, n) \kappa_{\beta \dot{\alpha}}(1, n)
$$

valid for this helicity configuration.

The solution to $(2.16)$ is [1]

$$
\begin{gathered}
\mathcal{J}_{\alpha \dot{\alpha}}\left(1^{+}, \ldots, n^{+}\right)=u_{\alpha}(h) u^{\beta}(h) \kappa_{\beta \dot{\alpha}}(1, n) X\left(1^{+}, \ldots, n^{+}\right), \\
X\left(1^{+}, \ldots, n^{+}\right)=\frac{(-\sqrt{2})^{n-1}}{\langle h|1, \ldots, n| h\rangle}
\end{gathered}
$$

for the case where all of the gluons have the same helicity.

\subsection{The quark current}

We now review the fermion currents presented by Berends and Giele [1]. These currents consist of a quark line plus $n$ gluons in the tree approximation. 
One end of the quark line will be off shell. In the following, all momenta flow into the diagram. We will denote the momentum of the quark by $p$ and its color index by $i$. The antiquark will have momentum $q$ and color index $j$. In the case where the quark is off shell we have, by definition:

$$
\begin{aligned}
\widehat{\bar{\psi}}_{j i}(q ; 1, \ldots, n)= & \sum_{\mathcal{P}(1 \ldots n)} \sum_{\ell=0}^{n-1} \frac{1}{\ell !(n-\ell) !} \widehat{\bar{\psi}}_{j m}(q ; 1, \ldots, \ell) \\
& \times(-i g)\left(T^{x}\right)_{m i} \gamma^{\xi} \widehat{J}_{\xi}^{x}(\ell+1, \ldots, n) \frac{-i}{\not q+\not(1, n)} .
\end{aligned}
$$

This simplifies to

$$
\widehat{\bar{\psi}}_{j i}(q ; 1, \ldots, n)=g^{n} \sum_{\mathcal{P}(1 \ldots n)}(\Omega[1, n])_{j i} \bar{\psi}(q ; 1, \ldots, n)
$$

where

$$
\begin{aligned}
& \bar{\psi}(q ; 1, \ldots, n)= \\
& \quad=\frac{-1}{[q+\kappa(1, n)]^{2}} \sum_{\ell=0}^{n-1} \bar{\psi}(q ; 1, \ldots, \ell) \not \supset(\ell+1, \ldots, n)[\not 1+\kappa(1, n)] .
\end{aligned}
$$

Since the helicity of the quark line is conserved in the massless limit, (2.19b) has two translations to Weyl spinors:

$$
\begin{aligned}
& \bar{\psi}^{\alpha}\left(q^{-} ; 1, \ldots, n\right)= \\
& \quad=\frac{-\sqrt{2}}{[q+\kappa(1, n)]^{2}} \sum_{\ell=0}^{n-1} \bar{\psi}^{\beta}\left(q^{-} ; 1, \ldots, \ell\right) \mathcal{J}_{\beta \dot{\alpha}}(\ell+1, \ldots, n)[\bar{q}+\bar{\kappa}(1, n)]^{\dot{\alpha} \alpha}
\end{aligned}
$$

for a left-handed antiquark and

$$
\begin{aligned}
& \bar{\psi}_{\dot{\alpha}}\left(q^{+} ; 1, \ldots, n\right)= \\
& \quad=\frac{-\sqrt{2}}{[q+\kappa(1, n)]^{2}} \sum_{\ell=0}^{n-1} \bar{\psi}_{\dot{\beta}}\left(q^{+} ; 1, \ldots, \ell\right) \overline{\mathcal{J}}^{\dot{\beta} \alpha}(\ell+1, \ldots, n)[q+\kappa(1, n)]_{\alpha \dot{\alpha}}
\end{aligned}
$$

for a right-handed antiquark.

In analogous fashion, when the antiquark is off shell we have

$$
\begin{aligned}
\widehat{\psi}_{j i}(1, \ldots, n ; p)= & \sum_{\mathcal{P}(1 \ldots n)} \sum_{\ell=1}^{n} \frac{1}{\ell !(n-\ell) !} \frac{i}{\not k(1, n)+\not p} \\
& \times(-i g)\left(T^{x}\right)_{j m} \gamma^{\xi} \widehat{J}_{\xi}^{x}(1, \ldots, \ell) \widehat{\psi}_{m i}(\ell+1, \ldots, n ; p),
\end{aligned}
$$


which simplifies to

$$
\widehat{\psi}_{j i}(1, \ldots, n ; p)=g^{n} \sum_{\mathcal{P}(1 \ldots n)}(\Omega[1, n])_{j i} \psi(1, \ldots, n ; p),
$$

where

$$
\begin{aligned}
& \psi(1, \ldots, n ; p)= \\
& \quad=\frac{1}{[\kappa(1, n)+p]^{2}} \sum_{\ell=1}^{n}\left[k^{\prime}(1, n)+\not p\right] \not \partial(1, \ldots, \ell) \psi(\ell+1, \ldots, n ; p) .
\end{aligned}
$$

In terms of Weyl spinors, (2.22b) reads:

$$
\begin{aligned}
& \psi_{\alpha}\left(1, \ldots, n ; p^{-}\right)= \\
& \quad=\frac{\sqrt{2}}{[\kappa(1, n)+p]^{2}} \sum_{\ell=1}^{n}[\kappa(1, n)+p]_{\alpha \dot{\alpha}} \overline{\mathcal{J}}^{\dot{\alpha} \beta}(1, \ldots, \ell) \psi_{\beta}\left(\ell+1, \ldots, n ; p^{-}\right)
\end{aligned}
$$

for a left-handed quark and

$$
\begin{aligned}
& \psi^{\dot{\alpha}}\left(1, \ldots, n ; p^{+}\right)= \\
& \quad=\frac{\sqrt{2}}{[\kappa(1, n)+p]^{2}} \sum_{\ell=1}^{n}[\bar{\kappa}(1, n)+\bar{p}]^{\dot{\alpha} \alpha} \mathcal{J}_{\alpha \dot{\beta}}(1, \ldots, \ell) \psi^{\dot{\beta}}\left(\ell+1, \ldots, n ; p^{+}\right)
\end{aligned}
$$

for a right-handed quark. It is worth mentioning that

$$
\psi^{\dot{\alpha}}\left(1, \ldots, n ; p^{+}\right)=(-1)^{n} \varepsilon^{\dot{\alpha} \dot{\beta}} \bar{\psi}_{\dot{\beta}}\left(p^{+} ; n, n-1, \ldots, 1\right)
$$

and

$$
\psi_{\alpha}\left(1, \ldots, n ; p^{-}\right)=(-1)^{n} \bar{\psi}^{\beta}\left(p^{-} ; n, n-1, \ldots, 1\right) \varepsilon_{\beta \alpha} .
$$

The recursion relations (2.20) and (2.23) are easily solved when all of the gluons have the same helicity. The gauge choice for the gluons is the same as that used for the pure gluonic current, namely (2.13). The solutions for $n \geq 1$ are

$$
\bar{\psi}^{\alpha}\left(q^{-} ; 1^{+}, \ldots, n^{+}\right)=-\sqrt{2} u^{\alpha}(q)\langle q h\rangle \frac{\langle h 1\rangle}{\langle q 1\rangle} X\left(1^{+}, \ldots, n^{+}\right)
$$

and

$$
\bar{\psi}_{\dot{\alpha}}\left(q^{+} ; 1^{+}, \ldots, n^{+}\right)=\sqrt{2} u^{\beta}(h)[q+\kappa(1, n)]_{\beta \dot{\alpha}} \frac{\langle h 1\rangle}{\langle q 1\rangle} X\left(1^{+}, \ldots, n^{+}\right),
$$


where the scalar function $X\left(1^{+}, \ldots, n^{+}\right)$is given by $(2.17 \mathrm{~b})$. For $n=0$ we have simply

$$
\begin{aligned}
& \bar{\psi}^{\alpha}\left(q^{-}\right)=u^{\alpha}(q), \\
& \bar{\psi}_{\dot{\alpha}}\left(q^{+}\right)=\bar{u}_{\dot{\alpha}}(q),
\end{aligned}
$$

consistent with (2.25) and (2.26). The off-shell antiquark currents are easily obtained from (2.24).

\section{CURRENTS WITH SEVERAL OFF-SHELL GLUONS}

In this section we will begin by presenting a generalization of the Berends and Giele gluon recursion relation [1] which allows two or more of the gluons to be off shell. We will then specialize to the situation in which one gluon is off shell, one gluon has a "generalized" polarization spinor, and the remaining gluons are on shell. If all of the on-shell gluons have the same helicity, the generalized recursion relation simplifies, and we are able to solve it for arbitrary $n$. As we shall see in Section 4, this quantity may be used to aid in the computation of the process (1.1).

\subsection{The recursion relation}

We define the current $\widehat{I}_{\xi}^{x}\left(1^{*}, 2^{*}, \ldots, n^{*}\right)$ to consist of the sum of all tree graphs with $n+1$ external gluons. The gluons labelled by $\{1,2, \ldots, n\}$ have momenta $k_{1}, k_{2}, \ldots, k_{n}$, color indices $a_{1}, a_{2}, \ldots, a_{n}$, and special polarization vectors $\epsilon\left(1^{*}\right), \epsilon\left(2^{*}\right), \ldots, \epsilon\left(n^{*}\right)$. These special polarizations are included to avoid a proliferation of indices: in general,

$$
k_{j} \cdot \epsilon(j) \neq 0 .
$$


Furthermore, we allow for

$$
k_{j}^{2} \neq 0
$$

We have not included the propagator factors for theses gluons. The last gluon has momentum $k_{n+1}=-\kappa(1, n)$, color index $x$, and Lorentz index $\xi$. We $d o$ include the propagator for this gluon in the definition of $\widehat{I}$.

Because of (3.1) and (3.2), it is easy to obtain currents with any number of off-shell gluons from $\widehat{I}_{\xi}^{x}\left(1^{*}, 2^{*}, \ldots, n^{*}\right)$. All that is required is to remove the appropriate polarization vectors, and supply a propagator for each off-shell gluon. "Normal" polarization vectors may be used to replace the polarizations of the remaining gluons which are to be on shell.

An examination of the derivation given by Berends and Giele [1] for the gluon recursion relation (2.7) reveals that the kinematic and color structures factorize quite early in the derivation. As a result, it is immediately obvious that $\widehat{I}$ satisfies the same factorization property as $\widehat{J}$ and the recursion relation for $\widehat{I}$ has the same form as (2.7), namely,

$$
\begin{aligned}
& \widehat{I}_{\xi}^{x}\left(1^{*}, \ldots, n^{*}\right)=2 g^{n-1} \sum_{\mathcal{P}(1 \ldots n)} \operatorname{tr}\left(\Omega[1, n] T^{x}\right) I_{\xi}\left(1^{*}, \ldots, n^{*}\right), \\
& I_{\xi}\left(1^{*}, \ldots, n^{*}\right)=\frac{1}{\kappa^{2}(1, n)}\left(\sum_{j=1}^{n-1} \llbracket I\left(1^{*}, \ldots, j^{*}\right), I\left((j+1)^{*}, \ldots, n^{*}\right) \rrbracket \xi\right. \\
& \left.+\sum_{j=1}^{n-2} \sum_{\ell=j+1}^{n-1}\left\{I\left(1^{*}, \ldots, j^{*}\right), I\left((j+1)^{*}, \ldots, \ell^{*}\right), I\left((\ell+1)^{*}, \ldots, n^{*}\right)\right\}_{\xi}\right), \\
& I\left(1^{*}\right)=\epsilon\left(1^{*}\right)
\end{aligned}
$$


The only difference between $(3.3 \mathrm{~b})$ and $(2.7 \mathrm{~b})$ is the appearance of a new square bracket function, defined by

$$
\begin{aligned}
& \llbracket I\left(1^{*}, \ldots, j^{*}\right), I\left((j+1)^{*}, \ldots, n^{*}\right) \rrbracket \xi \\
& =[2 \kappa(j+1, n)+\kappa(1, j)] \cdot I\left(1^{*}, \ldots, j^{*}\right) I_{\xi}\left((j+1)^{*}, \ldots, n^{*}\right) \\
& \quad-[2 \kappa(1, j)+\kappa(j+1, n)] \cdot I\left((j+1)^{*}, \ldots, n^{*}\right) I_{\xi}\left(1^{*}, \ldots, j^{*}\right) \\
& \quad+I\left(1^{*}, \ldots, j^{*}\right) \cdot I\left((j+1)^{*}, \ldots, n^{*}\right)[\kappa(1, j)-\kappa(j+1, n)]_{\xi}
\end{aligned}
$$

The curly bracket function remains as in (2.10). A comparison of (3.4) with (2.15) reveals that the differences between the two are generated by the fact that $I\left(1^{*}, \ldots, n^{*}\right)$ is not a conserved current, that is

$$
\kappa(1, n) \cdot I\left(1^{*}, \ldots, n^{*}\right) \neq 0
$$

In spite of the loss of current conservation, $I$ still satisfies the following properties:

$$
\begin{gathered}
I\left(1^{*}, 2^{*}, \ldots, n^{*}\right)=(-1)^{n} I\left(n^{*}, \ldots, 2^{*}, 1^{*}\right), \\
\sum_{\mathcal{C}(1 \ldots n)} I\left(1^{*}, \ldots, n^{*}\right)=0, \\
\sum_{j=1}^{n} I\left(2^{*}, \ldots, j^{*}, 1^{*},(j+1)^{*}, \ldots, n^{*}\right)=0,
\end{gathered}
$$

corresponding to (2.11a)-(2.11c).

\subsection{The modified gluon current}

We will now consider $\mathcal{I}_{\alpha \dot{\alpha}}\left(1^{*}, 2^{+}, \ldots, n^{+}\right)$, a current with just one "generalized" polarization vector and $n-1$ like-helicity on-shell gluons. The polarization spinors for the gluons labelled $2,3, \ldots, n$ are given by (2.13). The "generalized" polarization spinor for the first gluon is defined to be

$$
\mathcal{I}_{\alpha \dot{\alpha}}\left(1^{*}\right) \equiv u_{\alpha}(h) u^{\beta}(h) k_{1 \beta \dot{\alpha}}
$$


with

$$
k_{1}^{2} \neq 0
$$

As we shall see in Section 4, this form of $\mathcal{I}\left(1^{*}\right)$ appears when we consider the computation of the process (1.1). Even though $k_{1}^{2}$ does not vanish, we still have the relation

$$
\bar{k}_{1}^{\dot{\alpha} \alpha} \mathcal{I}_{\alpha \dot{\alpha}}\left(1^{*}\right)=0
$$

as if $\mathcal{I}\left(1^{*}\right)$ were a true polarization spinor. Because of (3.9), and since all of the other gluons have "normal" polarizations, $\mathcal{I}\left(1^{*}, 2^{+}, \ldots, n^{+}\right)$is a conserved current, in contrast to the more general case leading to (3.5). Thus, the square bracket function in (3.4) reduces to the form (2.15). Moreover, $\mathcal{I}\left(1^{*}\right)$ is proportional to $u_{\alpha}(h)$. This means that key properties ( $c f$. equations $(2.12),(2.14)$, and (2.15)) leading to the simplified form of the recursion relation (2.16) for $\mathcal{J}$ still hold when one of the $\mathcal{J}$ 's is replaced by an $\mathcal{I}$. Thus,

$$
\begin{aligned}
& \mathcal{I}_{\alpha \dot{\alpha}}\left(1^{*}, 2^{+}, \ldots, n^{+}\right)= \\
& \quad=\frac{-\sqrt{2}}{\kappa^{2}(1, n)} \sum_{j=1}^{n-1} \mathcal{I}_{\alpha \dot{\beta}}\left(1^{*}, 2^{+}, \ldots, j^{+}\right) \overline{\mathcal{J}}^{\dot{\beta} \beta}\left((j+1)^{+}, \ldots, n^{+}\right) \kappa_{\beta \dot{\alpha}}(1, n),
\end{aligned}
$$

with (3.7) giving the starting point $\mathcal{I}\left(1^{*}\right)$.

The next current, $\mathcal{I}\left(1^{*}, 2^{+}\right)$, is found by direct calculation from (3.10) to be

$$
\mathcal{I}_{\alpha \dot{\alpha}}\left(1^{*}, 2^{+}\right)=-\sqrt{2} \frac{u_{\alpha}(h) u^{\beta}(h)\left(k_{1}+k_{2}\right)_{\beta \dot{\alpha}}}{\langle 2 h\rangle} \frac{u^{\gamma}(h) k_{1 \gamma \dot{\gamma}} \bar{u}^{\dot{\gamma}}\left(k_{2}\right)}{\left(k_{1}+k_{2}\right)^{2}},
$$

which should be compared to

$$
\mathcal{J}_{\alpha \dot{\alpha}}\left(1^{+}, 2^{+}\right)=-\sqrt{2} \frac{u_{\alpha}(h) u^{\beta}(h)\left(k_{1}+k_{2}\right)_{\beta \dot{\alpha}}}{\langle 2 h\rangle} \frac{1}{\langle h 1\rangle\langle 12\rangle},
$$

as obtained from $(2.17)$. If it were true that $k_{1}^{2}=0$, then we would have

$$
\frac{u^{\gamma}(h) k_{1 \gamma \dot{\gamma}} \bar{u}^{\dot{\gamma}}\left(k_{2}\right)}{\left(k_{1}+k_{2}\right)^{2}}=\frac{\langle h 1\rangle\langle 21\rangle^{*}}{\langle 21\rangle\langle 21\rangle^{*}}=\frac{\langle h 1\rangle}{\langle 21\rangle} .
$$


Thus, we see that

$$
\lim _{k_{1}^{2} \rightarrow 0} \mathcal{I}_{\alpha \dot{\alpha}}\left(1^{*}, 2^{+}\right)=\langle h|1| h\rangle \mathcal{J}_{\alpha \dot{\alpha}}\left(1^{+}, 2^{+}\right) .
$$

Equation (3.14) suggests that we try the following ansatz for $\mathcal{I}\left(1^{*}, 2^{+}, \ldots, n^{+}\right)$, valid for $n \geq 2$ :

$$
\begin{aligned}
& \mathcal{I}_{\alpha \dot{\alpha}}\left(1^{*}, 2^{+}, \ldots, n^{+}\right)= \\
& \quad=(-\sqrt{2})^{n-1} \frac{u_{\alpha}(h) u^{\beta}(h) \kappa_{\beta \dot{\alpha}}(1, n)}{\langle 2|3, \ldots, n| h\rangle}\left[\frac{u^{\gamma}(h) k_{1 \gamma \dot{\gamma}} \bar{u} \dot{\gamma}\left(k_{2}\right)}{\left(k_{1}+k_{2}\right)^{2}}+k_{1}^{2} \Lambda_{n}\right] .
\end{aligned}
$$

The first term of $(3.15)$ reduces to $\langle h|1| h\rangle \mathcal{J}_{\alpha \dot{\alpha}}\left(1^{+}, 2^{+}, \ldots, n^{+}\right)$in the limit $k_{1}^{2} \rightarrow 0$, while the second term, with its undetermined function $\Lambda_{n}$, vanishes in that limit. It is obvious from (3.12) that

$$
\Lambda_{2}=0
$$

We now prove (3.15) by mathematical induction. Assume that (3.15) is true for $\mathcal{I}\left(1^{*}, 2^{+}, \ldots, \ell^{+}\right)$with $\ell<n$. The recursion relation (3.10) along with the known solution (2.17) for $\mathcal{J}$ tell us that

$$
\begin{aligned}
\mathcal{I}_{\alpha \dot{\alpha}}\left(1^{*}, 2^{+}, \ldots, n^{+}\right)= & \\
=- & \sqrt{2}\left[\mathcal{I}_{\alpha \dot{\beta}}\left(1^{*}\right) \overline{\mathcal{J}}^{\dot{\beta} \beta}\left(2^{+}, \ldots, n^{+}\right)\right. \\
& \left.\quad+\sum_{\ell=2}^{n-1} \mathcal{I}_{\alpha \dot{\beta}}\left(1^{*}, 2^{+}, \ldots, \ell^{+}\right) \overline{\mathcal{J}}^{\dot{\beta} \beta}\left((\ell+1)^{+}, \ldots, n^{+}\right)\right] \frac{\kappa_{\beta \dot{\alpha}}(1, n)}{\kappa^{2}(1, n)} \\
=\frac{-(-\sqrt{2})^{n-1}}{\kappa^{2}(1, n)} \frac{u_{\alpha}(h) u^{\beta}(h) \kappa_{\beta \dot{\alpha}}(1, n)}{\langle 2|3, \ldots, n| h\rangle}\left\{\frac{u^{\delta}(h) k_{1 \delta \dot{\delta}} \bar{\kappa}^{\dot{\delta} \gamma}(2, n) u_{\gamma}(h)}{\langle h 2\rangle}\right. & \\
& +\sum_{\ell=2}^{n-1} \frac{\langle\ell \ell+1\rangle}{\langle\ell|h| \ell+1\rangle} u^{\delta}(h) \kappa_{\delta \dot{\delta}}(1, \ell) \bar{\kappa}^{\dot{\delta} \epsilon}(\ell+1, n) u_{\epsilon}(h) \frac{u^{\gamma}(h) k_{1 \gamma \dot{\gamma}} \bar{u}^{\dot{\gamma}}\left(k_{2}\right)}{\left(k_{1}+k_{2}\right)^{2}} \\
& \left.+\sum_{\ell=2}^{n-1} \frac{\langle\ell \ell+1\rangle}{\langle\ell|h| \ell+1\rangle} u^{\delta}(h) \kappa_{\delta \dot{\delta}}(1, \ell) \bar{\kappa}^{\dot{\delta} \epsilon}(\ell+1, n) u_{\epsilon}(h) k_{1}^{2} \Lambda_{\ell}\right\} .
\end{aligned}
$$

Let us denote the second term in the curly brackets of (3.17) by $S$. Writing out the implicit $\kappa$-sums we have

$$
S=\frac{u^{\gamma}(h) k_{1 \gamma \dot{\gamma}} \bar{u}^{\dot{\gamma}}\left(k_{2}\right)}{\left(k_{1}+k_{2}\right)^{2}} \sum_{\ell=2}^{n-1} \sum_{i=1}^{\ell} \sum_{j=\ell+1}^{n} \frac{\langle\ell \ell+1\rangle}{\langle\ell|h| \ell+1\rangle} u^{\delta}(h) k_{i \delta} \bar{k}_{j}^{\dot{\delta} \epsilon} u_{\epsilon}(h) .
$$


Because $k_{1}^{2} \neq 0$,

$$
k_{1 \delta \dot{\delta}} \neq u_{\delta}\left(k_{1}\right) \bar{u}_{\dot{\delta}}\left(k_{1}\right)
$$

and we must treat the $i=1$ portion of (3.18) separately. Doing this and changing the order of the summations produces

$$
\begin{aligned}
S=\frac{u^{\gamma}(h) k_{1 \gamma \dot{\gamma}} \bar{u}^{\dot{\gamma}}\left(k_{2}\right)}{\left(k_{1}+k_{2}\right)^{2}} & {\left[\sum_{i=2}^{n-1} \sum_{j=i+1}^{n} \sum_{\ell=i}^{j-1} \frac{\langle\ell \ell+1\rangle}{\langle\ell|h| \ell+1\rangle}\langle h i\rangle\langle j i\rangle^{*}\langle j h\rangle\right.} \\
& \left.+\sum_{j=3}^{n} \sum_{\ell=2}^{j-1} \frac{\langle\ell \ell+1\rangle}{\langle\ell|h| \ell+1\rangle} u^{\delta}(h) k_{1 \delta \dot{\delta}} \dot{u}^{\dot{\delta}}\left(k_{j}\right)\langle j h\rangle\right] .
\end{aligned}
$$

The sums on $\ell$ may be performed using (A.16), with the result

$$
\begin{aligned}
S= & \frac{u^{\gamma}(h) k_{1 \gamma \dot{\gamma}} \bar{u}^{\dot{\gamma}}\left(k_{2}\right)}{\left(k_{1}+k_{2}\right)^{2}}\left[\sum_{i=2}^{n-1} \sum_{j=i+1}^{n} \frac{\langle i j\rangle}{\langle i|h| j\rangle}\langle h i\rangle\langle j i\rangle^{*}\langle j h\rangle\right. \\
& \left.+\sum_{j=3}^{n} \frac{\langle 2 j\rangle}{\langle 2|h| j\rangle} u^{\delta}(h) k_{1 \delta \dot{\delta}} \bar{u}^{\dot{\delta}}\left(k_{j}\right)\langle j h\rangle\right] \\
= & \frac{u^{\gamma}(h) k_{1 \gamma \dot{\gamma}} \bar{u}^{\dot{\gamma}}\left(k_{2}\right)}{\left(k_{1}+k_{2}\right)^{2}}\left[-\kappa^{2}(2, n)-\frac{u^{\delta}(h) k_{1 \delta \dot{\delta}} \bar{\kappa}(3, n)^{\dot{\delta} \epsilon} u_{\epsilon}\left(k_{2}\right)}{\langle h 2\rangle}\right] .
\end{aligned}
$$

The Weyl equation allows us to extend the sum in the numerator in the second term of $(3.21)$ to $\kappa(2, n)$. We may then write $k_{1}=\kappa(1, n)-\kappa(2, n)$ to obtain

$$
\begin{aligned}
-u^{\delta}(h) k_{1 \delta \dot{\delta}} \bar{\kappa}(3, n)^{\dot{\delta} \epsilon} u_{\epsilon}\left(k_{2}\right)= & -u^{\delta}(h) \kappa_{\delta \dot{\delta}}(1, n) \bar{\kappa}(2, n)^{\dot{\delta} \epsilon} u_{\epsilon}\left(k_{2}\right) \\
& +\langle h 2\rangle \kappa^{2}(2, n)
\end{aligned}
$$

Substituting (3.22) into (3.21) produces

$$
\begin{aligned}
S & =-\frac{u^{\gamma}(h) k_{1 \gamma \dot{\gamma}} \bar{u}^{\dot{\gamma}}\left(k_{2}\right)}{\left(k_{1}+k_{2}\right)^{2}} \frac{u^{\delta}(h) \kappa_{\delta \dot{\delta}}(1, n) \bar{\kappa}(2, n)^{\dot{\delta} \epsilon} u_{\epsilon}\left(k_{2}\right)}{\langle h 2\rangle} \\
& =-\frac{u^{\delta}(h) \kappa_{\delta \dot{\delta}}(1, n) \bar{\kappa}^{\dot{\delta} \epsilon}(2, n) k_{2 \epsilon \dot{\gamma}} \bar{k}_{1}^{\dot{\gamma} \gamma} u_{\gamma}(h)}{\langle h 2\rangle\left(k_{1}+k_{2}\right)^{2}}
\end{aligned}
$$


Repeated use of this trick yields

$$
\begin{aligned}
S= & -\frac{u^{\delta}(h) \kappa_{\delta \dot{\delta}}(1, n) \bar{\kappa}^{\dot{\delta} \epsilon}(2, n) k_{2 \epsilon \dot{\gamma}}\left(\bar{k}_{1}+\bar{k}_{2}\right) \dot{\gamma} u_{\gamma}(h)}{\langle h 2\rangle\left(k_{1}+k_{2}\right)^{2}} \\
= & -\frac{u^{\delta}(h) \kappa_{\delta \dot{\delta}}(1, n) \bar{\kappa}^{\dot{\delta} \gamma}(2, n) u_{\gamma}(h)}{\langle h 2\rangle} \\
& +\frac{u^{\delta}(h) \kappa_{\delta \dot{\delta}}(1, n) \bar{\kappa}^{\dot{\delta} \epsilon}(2, n) k_{1 \epsilon \dot{\gamma}}\left(\bar{k}_{1}+\bar{k}_{2}\right)^{\dot{\gamma} \gamma} u_{\gamma}(h)}{\langle h 2\rangle\left(k_{1}+k_{2}\right)^{2}} \\
= & -\frac{u^{\delta}(h) k_{1 \delta \dot{\delta}} \bar{\kappa}^{\dot{\delta} \gamma}(2, n) u_{\gamma}(h)}{\langle h 2\rangle} \\
& -\kappa^{2}(1, n) \frac{u^{\delta}(h) k_{1 \delta \dot{\delta}} \bar{u}^{\delta}\left(k_{2}\right)}{\left(k_{1}+k_{2}\right)^{2}} \\
& -k_{1}^{2} \frac{u^{\delta}(h) \kappa_{\delta \dot{\gamma}}(1, n)\left(\bar{k}_{1}+\bar{k}_{2}\right)^{\dot{\gamma} \gamma} u_{\gamma}(h)}{\langle h 2\rangle\left(k_{1}+k_{2}\right)^{2}} .
\end{aligned}
$$

By inserting (3.24) back into (3.17) we arrive at

$$
\begin{aligned}
& \mathcal{I}_{\alpha \dot{\alpha}}\left(1^{*}, 2^{+}, \ldots, n^{+}\right)= \\
&=\frac{(-\sqrt{2})^{n-1} u_{\alpha}(h) u^{\beta}(h) \kappa_{\beta \dot{\alpha}}(1, n)}{\langle 2|3, \ldots, n| h\rangle}\left\{\frac{u^{\delta}(h) k_{1 \delta \dot{\delta}} \bar{u}^{\delta}\left(k_{2}\right)}{\left(k_{1}+k_{2}\right)^{2}}\right. \\
& \quad+\frac{k_{1}^{2}}{\kappa^{2}(1, n)}\left[\frac{u^{\delta}(h) \kappa_{\delta \dot{\gamma}}(1, n)\left(\bar{k}_{1}+\bar{k}_{2}\right)^{\dot{\gamma} \gamma} u_{\gamma}(h)}{\langle h 2\rangle\left(k_{1}+k_{2}\right)^{2}}\right. \\
&\left.\left.\quad-\sum_{\ell=2}^{n-1} \Lambda_{\ell} \frac{\langle\ell \ell+1\rangle}{\langle\ell|h| \ell+1\rangle} u^{\delta}(h) \kappa_{\delta \dot{\delta}}(1, \ell) \bar{\kappa}^{\dot{\delta} \epsilon}(\ell+1, n) u_{\epsilon}(h)\right]\right\}
\end{aligned}
$$

which satisfies the ansatz (3.15) if we insist upon the following recursion relation for $\Lambda_{n}$ :

$$
\begin{aligned}
\Lambda_{n}=\frac{1}{\kappa^{2}(1, n)}[ & \frac{u^{\delta}(h) \kappa_{\delta \dot{\gamma}}(1, n)\left(\bar{k}_{1}+\bar{k}_{2}\right)^{\dot{\gamma} \gamma} u_{\gamma}(h)}{\langle h 2\rangle\left(k_{1}+k_{2}\right)^{2}} \\
& \left.-\sum_{\ell=2}^{n-1} \Lambda_{\ell} \frac{\langle\ell \ell+1\rangle}{\langle\ell|h| \ell+1\rangle} u^{\delta}(h) \kappa_{\delta \dot{\delta}}(1, \ell) \bar{\kappa}^{\dot{\delta} \epsilon}(\ell+1, n) u_{\epsilon}(h)\right] .
\end{aligned}
$$

Since $\Lambda_{2}$ vanishes, (3.26) tells us that

$$
\Lambda_{3}=\frac{u^{\delta}(h) k_{3 \delta \dot{\gamma}}\left(\bar{k}_{1}+\bar{k}_{2}\right)^{\dot{\gamma} \gamma} u_{\gamma}(h)}{\langle h 2\rangle\left(k_{1}+k_{2}\right)^{2}\left(k_{1}+k_{2}+k_{3}\right)^{2}} .
$$

Use of (3.27) in (3.26) to obtain $\Lambda_{4}$ yields

$$
\begin{aligned}
& \Lambda_{4}=\frac{u^{\delta}(h) \kappa_{\delta \dot{\gamma}}(1,4) \bar{\kappa}^{\dot{\gamma} \gamma}(1,2) u_{\gamma}(h)}{\langle h 2\rangle \kappa^{2}(1,2) \kappa^{2}(1,4)} \\
& +\frac{u^{\delta}(h) \kappa_{\delta \dot{\delta}}(1,3) \bar{k}_{4}^{\dot{\delta} \gamma} k_{3 \gamma \dot{\gamma}} \bar{\kappa}^{\dot{\gamma} \epsilon}(1,2) u_{\epsilon}(h)}{\langle h 2\rangle \kappa^{2}(1,2) \kappa^{2}(1,3) \kappa^{2}(1,4)} .
\end{aligned}
$$


The numerator in the second term of (3.28) may be rewritten as follows:

$$
\begin{aligned}
\mathcal{N} \equiv & u^{\delta}(h) \kappa_{\delta \dot{\delta}}(1,3) \bar{k}_{4}^{\dot{\delta} \gamma} k_{3 \gamma \dot{\gamma}} \bar{\kappa}^{\dot{\gamma} \epsilon}(1,2) u_{\epsilon}(h) \\
= & u^{\delta}(h) \kappa_{\delta \dot{\delta}}(1,4) \bar{k}_{4}^{\dot{\delta} \gamma} \kappa_{\gamma \dot{\gamma}}(1,3) \bar{\kappa}^{\dot{\gamma} \epsilon}(1,2) u_{\epsilon}(h) \\
& -\kappa^{2}(1,2) u^{\delta}(h) \kappa_{\delta \dot{\delta}}(1,3) \bar{k}_{4}^{\dot{\delta} \gamma} u_{\gamma}(h) \\
= & \kappa^{2}(1,4) u^{\delta}(h) k_{3 \delta \dot{\gamma}} \bar{\kappa}^{\dot{\gamma} \epsilon}(1,2) u_{\epsilon}(h) \\
& -\kappa^{2}(1,3) u^{\delta}(h) \kappa_{\delta \dot{\delta}}(1,4) \bar{\kappa}^{\dot{\delta} \epsilon}(1,2) u_{\epsilon}(h) \\
& +\kappa^{2}(1,2) u^{\gamma}(h) k_{4 \gamma \dot{\delta}} \bar{\kappa}^{\dot{\delta} \delta}(1,3) u_{\delta}(h),
\end{aligned}
$$

where we have made repeated use of the Weyl equation, the antisymmetry of the spinor product, and added and subtracted terms as required to complete the squares. When (3.29) is combined with (3.28) we obtain

$$
\Lambda_{4}=\frac{u^{\delta}(h) k_{3 \delta \dot{\gamma}} \bar{\kappa}^{\dot{\gamma} \gamma}(1,2) u_{\gamma}(h)}{\langle h 2\rangle \kappa^{2}(1,2) \kappa^{2}(1,3)}+\frac{u^{\delta}(h) k_{4 \delta \dot{\gamma}} \bar{\kappa}^{\dot{\gamma} \gamma}(1,3) u_{\gamma}(h)}{\langle h 2\rangle \kappa^{2}(1,3) \kappa^{2}(1,4)} .
$$

Equations (3.27) and (3.30) suggest the ansatz

$$
\Lambda_{n}=\frac{1}{\langle h 2\rangle} \sum_{j=3}^{n} \frac{u^{\delta}(h) k_{j \delta \dot{\gamma}} \bar{\kappa}^{\dot{\gamma} \gamma}(1, j) u_{\gamma}(h)}{\kappa^{2}(1, j-1) \kappa^{2}(1, j)}
$$

We prove this ansatz by mathematical induction. Assuming $\Lambda_{\ell}$ to be given by (3.31) for $\ell<n, \Lambda_{n}$ is determined by (3.26) to be

$$
\begin{gathered}
\Lambda_{n}=\frac{u^{\delta}(h) \kappa_{\delta \dot{\gamma}}(1, n) \bar{\kappa}^{\dot{\gamma} \gamma}(1,2) u_{\gamma}(h)}{\langle h 2\rangle \kappa^{2}(1,2) \kappa^{2}(1, n)} \\
-\sum_{\ell=3}^{n-1} \sum_{j=3}^{\ell} \frac{\langle\ell \ell+1\rangle}{\langle\ell|h| \ell+1\rangle} \frac{u^{\delta}(h) \kappa_{\delta \dot{\delta}}(1, \ell) \bar{\kappa}^{\dot{\delta} \epsilon}(\ell+1, n) u_{\epsilon}(h)}{\langle h 2\rangle \kappa^{2}(1, n)} \\
\times \frac{u^{\beta}(h) k_{j \beta \dot{\gamma}} \bar{\kappa}^{\dot{\gamma} \gamma}(1, j) u_{\gamma}(h)}{\kappa^{2}(1, j-1) \kappa^{2}(1, j)} .
\end{gathered}
$$


Let us interchange the order of the summations in the second term of (3.32) and break $\kappa(1, \ell)$ into $\kappa(1, j)+\kappa(j+1, \ell)$ :

$$
\begin{aligned}
\Lambda_{n}= & \frac{u^{\delta}(h) \kappa_{\delta \dot{\gamma}}(1, n) \bar{\kappa}^{\dot{\gamma} \gamma}(1,2) u_{\gamma}(h)}{\langle h 2\rangle \kappa^{2}(1,2) \kappa^{2}(1, n)} \\
& -\sum_{j=3}^{n-1} \sum_{\ell=j}^{n-1} \frac{\langle\ell \ell+1\rangle}{\langle\ell|h| \ell+1\rangle} \frac{u^{\delta}(h) \kappa_{\delta \dot{\delta}}(1, j) \bar{\kappa}^{\dot{\delta} \epsilon}(\ell+1, n) u_{\epsilon}(h)}{\langle h 2\rangle \kappa^{2}(1, n)} \\
& \times \frac{u^{\beta}(h) k_{j \beta \dot{\gamma}} \bar{\kappa}^{\dot{\gamma} \gamma}(1, j) u_{\gamma}(h)}{\kappa^{2}(1, j-1) \kappa^{2}(1, j)} \\
& -\sum_{j=3}^{n-2} \sum_{\ell=j+1}^{n-1} \frac{\langle\ell \ell+1\rangle}{\langle\ell|h| \ell+1\rangle} \frac{u^{\delta}(h) \kappa_{\delta \dot{\delta}}(j+1, \ell) \bar{\kappa}^{\dot{\delta} \epsilon}(\ell+1, n) u_{\epsilon}(h)}{\langle h 2\rangle \kappa^{2}(1, n)} \\
& \times \frac{u^{\beta}(h) k_{j \beta \dot{\gamma}} \bar{\kappa}^{\dot{\gamma} \gamma}(1, j) u_{\gamma}(h)}{\kappa^{2}(1, j-1) \kappa^{2}(1, j)} .
\end{aligned}
$$

We have adjusted the limits in the third term of (3.33) to reflect the vanishing of $\kappa(j+1, \ell)$ for $j=\ell$. Denote the three contributions in $(3.33)$ by $\mathcal{Z}_{1}, \mathcal{Z}_{2}$, and $\mathcal{Z}_{3}$ respectively.

We examine $\mathcal{Z}_{3}$ first. Writing out the $\ell$-dependent $\kappa$-sums yields

$$
\begin{array}{r}
\mathcal{Z}_{3}=-\sum_{j=3}^{n-2} \sum_{\ell=j+1}^{n-1} \sum_{i=j+1}^{\ell} \sum_{m=\ell+1}^{n} \frac{\langle\ell \ell+1\rangle}{\langle\ell|h| \ell+1\rangle} \frac{u^{\delta}(h) k_{i \delta \dot{\delta}} \bar{k}_{m}^{\dot{\delta} \epsilon} u_{\epsilon}(h)}{\langle h 2\rangle \kappa^{2}(1, n)} \\
\times \frac{u^{\beta}(h) k_{j \beta \dot{\gamma}} \bar{\kappa}^{\dot{\gamma} \gamma}(1, j) u_{\gamma}(h)}{\kappa^{2}(1, j-1) \kappa^{2}(1, j)} .
\end{array}
$$

Next, we interchange the sum over $\ell$ with both the sum on $i$ and the sum on $m$ and use (A.16) to do the $\ell$-summation:

$$
\begin{array}{r}
\mathcal{Z}_{3}=-\sum_{j=3}^{n-2} \sum_{m=j+2}^{n} \sum_{i=j+1}^{m-1} \sum_{\ell=i}^{m-1} \frac{\langle\ell \ell+1\rangle}{\langle\ell|h| \ell+1\rangle} \frac{u^{\delta}(h) k_{i \delta \dot{\delta}} \bar{k}_{m}^{\dot{\delta} \epsilon} u_{\epsilon}(h)}{\langle h 2\rangle \kappa^{2}(1, n)} \\
\times \frac{u^{\beta}(h) k_{j \beta \dot{\gamma}} \bar{\kappa}^{\dot{\gamma} \gamma}(1, j) u_{\gamma}(h)}{\kappa^{2}(1, j-1) \kappa^{2}(1, j)} \\
=-\sum_{j=3} \sum_{m=j+2}^{n-2} \sum_{i=j+1}^{m-1} \frac{\langle i m\rangle}{\langle i|h| m\rangle} \frac{\langle h i\rangle\langle m i\rangle^{*}\langle m h\rangle}{\langle h 2\rangle \kappa^{2}(1, n)} \\
\times \frac{u^{\beta}(h) k_{j \beta} \bar{\gamma}^{\dot{\gamma} \gamma}(1, j) u_{\gamma}(h)}{\kappa^{2}(1, j-1) \kappa^{2}(1, j)} .
\end{array}
$$


Recognizing that $\langle i m\rangle\langle m i\rangle^{*}=-2 k_{i} \cdot k_{m}$ allows us to write (3.35) as

$$
\mathcal{Z}_{3}=\sum_{j=3}^{n-1} \frac{\kappa^{2}(j+1, n) u^{\beta}(h) k_{j \beta \dot{\gamma}} \bar{\kappa}^{\dot{\gamma} \gamma}(1, j) u_{\gamma}(h)}{\langle h 2\rangle \kappa^{2}(1, n) \kappa^{2}(1, j-1) \kappa^{2}(1, j)}
$$

where we have extended the sum to $j=n-1$ because $k_{n}^{2}=0$.

Setting $\mathcal{Z}_{3}$ aside, we turn to $\mathcal{Z}_{2}$. There is only one $\ell$-dependent $\kappa$-sum here, so we write

$$
\begin{gathered}
\mathcal{Z}_{2}=-\sum_{j=3}^{n-1} \sum_{\ell=j}^{n-1} \sum_{m=\ell+1}^{n} \frac{\langle\ell \ell+1\rangle}{\langle\ell|h| \ell+1\rangle} \frac{u^{\delta}(h) \kappa_{\delta \dot{\delta}}(1, j) \bar{k}_{m}^{\dot{\delta} \epsilon} u_{\epsilon}(h)}{\langle h 2\rangle \kappa^{2}(1, n)} \\
\times \frac{u^{\beta}(h) k_{j \beta \dot{\gamma}} \bar{\kappa}^{\dot{\gamma} \gamma}(1, j) u_{\gamma}(h)}{\kappa^{2}(1, j-1) \kappa^{2}(1, j)} .
\end{gathered}
$$

Before we can do the sum on $\ell$, we must interchange it with the sum on $m$. Once this is accomplished, we may use (A.16) to perform the sum:

$$
\begin{aligned}
\mathcal{Z}_{2} & =-\sum_{j=3}^{n-1} \sum_{m=j+1}^{n} \sum_{\ell=j}^{m-1} \frac{\langle\ell \ell+1\rangle}{\langle\ell|h| \ell+1\rangle} \frac{u^{\delta}(h) \kappa_{\delta \dot{\delta}}(1, j) \bar{k}_{m}^{\dot{\delta} \epsilon} u_{\epsilon}(h)}{\langle h 2\rangle \kappa^{2}(1, n)} \frac{u^{\beta}(h) k_{j \beta \dot{\gamma}} \bar{\kappa}^{\dot{\gamma} \gamma}(1, j) u_{\gamma}(h)}{\kappa^{2}(1, j-1) \kappa^{2}(1, j)} \\
& =-\sum_{j=3}^{n-1} \sum_{m=j+1}^{n} \frac{\langle j m\rangle}{\langle j|h| m\rangle} \frac{u^{\delta}(h) \kappa_{\delta \dot{\delta}}(1, j) \bar{u}^{\dot{\delta}}\left(k_{m}\right)\langle m h\rangle}{\langle h 2\rangle \kappa^{2}(1, n)} \frac{\langle h j\rangle \bar{u}_{\dot{\gamma}}\left(k_{j}\right) \bar{\kappa}^{\dot{\gamma} \gamma}(1, j) u_{\gamma}(h)}{\kappa^{2}(1, j-1) \kappa^{2}(1, j)} \\
& =\sum_{j=3}^{n-1} \frac{u^{\delta}(h) \kappa_{\delta \dot{\delta}}(1, j) \bar{\kappa}^{\dot{\delta} \beta}(j+1, n) k_{j \beta \dot{\gamma}} \bar{\kappa}^{\dot{\gamma} \gamma}(1, j) u_{\gamma}(h)}{\langle h 2\rangle \kappa^{2}(1, n) \kappa^{2}(1, j-1) \kappa^{2}(1, j)} .
\end{aligned}
$$

Writing $\kappa_{\delta \dot{\delta}}(1, j)=\kappa_{\delta \dot{\delta}}(1, n)-\kappa_{\delta \dot{\delta}}(j+1, n)$ yields

$$
\begin{aligned}
\mathcal{Z}_{2}= & \sum_{j=3}^{n-1} \frac{u^{\delta}(h) \kappa_{\delta \dot{\delta}}(1, n) \bar{\kappa}^{\dot{\delta} \beta}(j+1, n) k_{j \beta \dot{\gamma}} \bar{\kappa}^{\dot{\gamma} \gamma}(1, j) u_{\gamma}(h)}{\langle h 2\rangle \kappa^{2}(1, n) \kappa^{2}(1, j-1) \kappa^{2}(1, j)} \\
& -\sum_{j=3}^{n-1} \frac{\kappa^{2}(j+1, n) u^{\beta}(h) k_{j \beta \dot{\gamma}} \bar{\kappa}^{\dot{\gamma} \gamma}(1, j) u_{\gamma}(h)}{\langle h 2\rangle \kappa^{2}(1, n) \kappa^{2}(1, j-1) \kappa^{2}(1, j)} .
\end{aligned}
$$

We recognize the second term in $(3.39)$ as precisely $-\mathcal{Z}_{3}$ : hence, when we form $\mathcal{Z}_{23} \equiv \mathcal{Z}_{2}+\mathcal{Z}_{3}$ we are left with only

$$
\mathcal{Z}_{23}=\sum_{j=3}^{n-1} \frac{u^{\delta}(h) \kappa_{\delta \dot{\delta}}(1, n) \bar{\kappa}^{\dot{\delta} \beta}(j+1, n) k_{j \beta \dot{\gamma}} \bar{\kappa}^{\dot{\gamma} \gamma}(1, j) u_{\gamma}(h)}{\langle h 2\rangle \kappa^{2}(1, n) \kappa^{2}(1, j-1) \kappa^{2}(1, j)} .
$$


Let us call the numerator of $(3.40) \mathcal{N}_{23}$.

We may use the Weyl equation and the clever addition and subtraction of terms to write $\mathcal{N}_{23}$ as the combination of three terms containing perfect squares of momentum sums:

$$
\begin{aligned}
\mathcal{N}_{23}= & u^{\delta}(h) \kappa_{\delta \dot{\delta}}(1, n) \bar{\kappa}^{\dot{\delta} \beta}(j+1, n) k_{j \beta \dot{\gamma}} \bar{\kappa}^{\dot{\gamma} \gamma}(1, j) u_{\gamma}(h) \\
= & \kappa^{2}(1, n) u^{\delta}(h) k_{j \delta \dot{\gamma}} \bar{\kappa}^{\dot{\gamma} \gamma}(1, j) u_{\gamma}(h) \\
& -u^{\delta}(h) \kappa_{\delta \dot{\delta}}(1, n) \bar{\kappa}^{\dot{\delta} \beta}(1, j) k_{j \beta \dot{\gamma}} \bar{\kappa}^{\dot{\gamma} \gamma}(1, j-1) u_{\gamma}(h) \\
= & \kappa^{2}(1, n) u^{\delta}(h) k_{j \delta \dot{\gamma}} \bar{\kappa}^{\dot{\gamma} \gamma}(1, j) u_{\gamma}(h) \\
& -\kappa^{2}(1, j) u^{\delta}(h) \kappa_{\delta \dot{\delta}}(1, n) \bar{\kappa}^{\dot{\delta} \gamma}(1, j-1) u_{\gamma}(h) \\
& +\kappa^{2}(1, j-1) u^{\delta}(h) \kappa_{\delta \dot{\delta}}(1, n) \bar{\kappa}^{\dot{\delta} \beta}(1, j) u_{\beta}(h) .
\end{aligned}
$$

Insertion of (3.41) into (3.40) gives

$$
\begin{aligned}
\mathcal{Z}_{23}= & \sum_{j=3}^{n-1} \frac{u^{\delta}(h) k_{j \delta} \bar{\kappa}^{\dot{\gamma} \gamma}(1, j) u_{\gamma}(h)}{\langle h 2\rangle \kappa^{2}(1, j-1) \kappa^{2}(1, j)} \\
& -\sum_{j=3}^{n-1} \frac{u^{\delta}(h) \kappa_{\delta \dot{\delta}}(1, n)}{\langle h 2\rangle \kappa^{2}(1, n)}\left[\frac{\bar{\kappa}^{\dot{\delta} \gamma}(1, j-1) u_{\gamma}(h)}{\kappa^{2}(1, j-1)}-\frac{\bar{\kappa}^{\dot{\delta} \gamma}(1, j) u_{\gamma}(h)}{\kappa^{2}(1, j)}\right] .
\end{aligned}
$$

Most of the terms in the second sum in (3.42) cancel. The remainder reads

$$
\begin{aligned}
\mathcal{Z}_{23}= & \sum_{j=3}^{n-1} \frac{u^{\delta}(h) k_{j \delta \dot{\gamma}} \bar{\kappa}^{\dot{\gamma} \gamma}(1, j) u_{\gamma}(h)}{\langle h 2\rangle \kappa^{2}(1, j-1) \kappa^{2}(1, j)} \\
& -\frac{u^{\delta}(h) \kappa_{\delta \dot{\delta}}(1, n) \bar{\kappa}^{\dot{\delta} \gamma}(1,2) u_{\gamma}(h)}{\langle h 2\rangle \kappa^{2}(1,2) \kappa^{2}(1, n)}+\frac{u^{\delta}(h) \kappa_{\delta \dot{\delta}}(1, n) \bar{\kappa}^{\delta} \gamma}{\langle h 2\rangle \kappa^{2}(1, n-1) \kappa^{2}(1, n)} .
\end{aligned}
$$

If we compare the second term of $(3.43)$ to $\mathcal{Z}_{1}$ (i.e. the first term of $(3.33)$ ), we see that these two terms cancel. In addition, the last term of (3.43) provides a $j=n$ term for the sum appearing in the first term. Hence,

$$
\begin{aligned}
\Lambda_{n} & =\mathcal{Z}_{1}+\mathcal{Z}_{23} \\
& =\sum_{j=3}^{n} \frac{u^{\delta}(h) k_{j \delta \dot{\gamma}} \bar{\kappa}^{\dot{\gamma} \gamma}(1, j) u_{\gamma}(h)}{\langle h 2\rangle \kappa^{2}(1, j-1) \kappa^{2}(1, j)},
\end{aligned}
$$


proving the ansatz (3.31).

If we re-examine $(3.15)$, we see that the first term in the square brackets there corresponds to a $j=2$ term for the sum in $\Lambda_{n}$. We may thus combine (3.15) and (3.44) to produce the compact form

$$
\begin{aligned}
& \mathcal{I}_{\alpha \dot{\alpha}}\left(1^{*}, 2^{+}, \ldots, n^{+}\right)= \\
& \quad=(-\sqrt{2})^{n-1} k_{1}^{2} \frac{u_{\alpha}(h) u^{\beta}(h) \kappa_{\beta \alpha}(1, n)}{\langle h|2, \ldots, n| h\rangle} \sum_{j=2}^{n} \frac{u^{\delta}(h) k_{j \delta \dot{\gamma}} \dot{\kappa} \gamma \gamma(1, j) u_{\gamma}(h)}{\kappa^{2}(1, j-1) \kappa^{2}(1, j)},
\end{aligned}
$$

valid for $n \geq 2$.

\subsection{General Modified Gluon Currents}

In order to calculate the amplitude for the process (1.1), we require knowledge of $\mathcal{I}\left(1^{+}, 2^{*}, 3^{+}, \ldots, n^{+}\right), \mathcal{I}\left(1^{+}, 2^{+}, 3^{*}, 4^{+}, \ldots, n^{+}\right)$, etc. We begin by defining some useful notation.

We may separate the expression for $\mathcal{I}$ into a factor containing the spinor dependence and a scalar function. It is easily verified from the appropriate (modified) form of the recursion relation (2.16) that we may write

$$
\begin{aligned}
& \mathcal{I}_{\alpha \dot{\alpha}}\left(1^{+}, \ldots, m^{*}, \ldots, n^{+}\right)= \\
& \quad=(-\sqrt{2})^{n-1} u_{\alpha}(h) u^{\beta}(h) \kappa_{\beta \dot{\alpha}}(1, n) \mathcal{X}\left(1, \ldots, m^{*}, \ldots, n\right) .
\end{aligned}
$$

For the case already discussed, (3.45) tells us that

$$
\mathcal{X}\left(1^{*}, 2, \ldots, n\right)=\frac{-k_{1}^{2}}{\langle h|2, \ldots, n| h\rangle} \sum_{j=2}^{n} c(1,2, \ldots, j),
$$

where

$$
c(1,2, \ldots, j)=\frac{u^{\gamma}(h) \kappa_{\gamma \dot{\gamma}}(1, j) \bar{k}_{j}^{\dot{\gamma} \delta} u_{\delta}(h)}{\kappa^{2}(1, j-1) \kappa^{2}(1, j)} .
$$

Note that $c(1,2, \ldots, j)$ is symmetric in its first $j-1$ arguments.

We may obtain $\mathcal{X}\left(1,2^{*}, 3, \ldots, n\right)$ from the relation

$$
\begin{aligned}
-\mathcal{X}\left(1,2^{*}, 3, \ldots, n\right)= & \mathcal{X}\left(2^{*}, 1,3, \ldots, n\right) \\
& +\sum_{i=3}^{n} \mathcal{X}\left(2^{*}, 3, \ldots, i, 1, i+1, \ldots, n\right),
\end{aligned}
$$


a specialized form of (3.6c) with an overall factor removed. Careful consideration of (3.47) tells us that we have

$$
\begin{gathered}
\mathcal{X}\left(2^{*}, 1,3, \ldots, n\right)=\frac{-k_{2}^{2}}{\langle h|3, \ldots, n| h\rangle} \frac{\langle h 3\rangle}{\langle h|1| 3\rangle}\left[c(2,1)+\sum_{\ell=3}^{n} c(1,2, \ldots, \ell)\right], \\
\mathcal{X}\left(2^{*}, 3, \ldots, i, 1, i+1, \ldots, n\right)=\frac{-k_{2}^{2}}{\langle h|3, \ldots, n| h\rangle} \frac{\langle i i+1\rangle}{\langle i|1| i+1\rangle} \\
\times\left[\sum_{\ell=3}^{i} c(2,3, \ldots, \ell)+c(2,3, \ldots, i, 1)+\sum_{\ell=i+1}^{n} c(1,2, \ldots, \ell)\right] .
\end{gathered}
$$

Equation (3.50b) may even be used for $i=n$ if we define $k_{n+1} \equiv h$ for that purpose. Insertion of (3.50) into (3.49) yields

$$
\begin{aligned}
-\mathcal{X}\left(1,2^{*}, 3, \ldots, n\right)= \\
=\frac{-k_{2}^{2}}{\langle h|3, \ldots, n| h\rangle}\left[\frac{\langle h 3\rangle}{\langle h|1| 3\rangle} c(2,1)+\frac{\langle h 3\rangle}{\langle h|1| 3\rangle} \sum_{\ell=3}^{n} c(1,2, \ldots, \ell)\right. \\
\quad+\sum_{i=3}^{n} \sum_{\ell=3}^{i} \frac{\langle i i+1\rangle}{\langle i|1| i+1\rangle} c(2,3, \ldots, \ell)+\sum_{i=3}^{n} \frac{\langle i i+1\rangle}{\langle i|1| i+1\rangle} c(2,3, \ldots, i, 1) \\
\left.\quad+\sum_{i=3}^{n-1} \sum_{\ell=i+1}^{n} \frac{\langle i i+1\rangle}{\langle i|1| i+1\rangle} c(1,2, \ldots, \ell)\right] .
\end{aligned}
$$

The sums on $i$ appearing in double sums may be performed using (A.16). Reordering the double sums, doing the sum on $i$ in those terms, and changing the dummy summation variable from $i$ to $\ell$ in the fourth term gives

$$
\begin{aligned}
-\mathcal{X}\left(1,2^{*}, 3, \ldots, n\right)= \\
=\frac{-k_{2}^{2}}{\langle h|3, \ldots, n| h\rangle}\left[\frac{\langle h 3\rangle}{\langle h|1| 3\rangle} c(2,1)+\frac{\langle h 3\rangle}{\langle h|1| 3\rangle} \sum_{\ell=3}^{n} c(1,2, \ldots, \ell)\right. \\
\quad+\sum_{\ell=3}^{n} \frac{\langle\ell h\rangle}{\langle\ell|1| h\rangle} c(2,3, \ldots, \ell)+\sum_{\ell=3}^{n} \frac{\langle\ell \ell+1\rangle}{\langle\ell|1| \ell+1\rangle} c(2,3, \ldots, \ell, 1) \\
\left.\quad+\sum_{\ell=4}^{n} \frac{\langle 3 \ell\rangle}{\langle 3|1| \ell\rangle} c(1,2, \ldots, \ell)\right] .
\end{aligned}
$$

The last sum appearing in (3.52) is trivially extended to include $\ell=3$ since $\langle 33\rangle=0$. We then combine it with the second term in (3.52) by using (A.15):

$$
\frac{\langle h 3\rangle}{\langle h|1| 3\rangle}+\frac{\langle 3 \ell\rangle}{\langle 3|1| \ell\rangle}=\frac{\langle h \ell\rangle}{\langle h|1| \ell\rangle} .
$$


We split up the coefficient of $c(2,3, \ldots, \ell, 1)$ by using (A.15) in reverse:

$$
\frac{\langle\ell \ell+1\rangle}{\langle\ell|1| \ell+1\rangle}=\frac{\langle\ell h\rangle}{\langle\ell|1| h\rangle}+\frac{\langle h \ell+1\rangle}{\langle h|1| \ell+1\rangle} \text {. }
$$

Incorporation of the changes to (3.52) implied by (3.53) and (3.54) produces:

$$
\begin{aligned}
-\mathcal{X}\left(1,2^{*}, 3, \ldots, n\right)= & \\
= & \frac{-k_{2}^{2}}{\langle h|3, \ldots, n| h\rangle}\left[\frac{\langle h 3\rangle}{\langle h|1| 3\rangle} c(2,1)+\sum_{\ell=3}^{n} \frac{\langle h \ell\rangle}{\langle h|1| \ell\rangle} c(1,2, \ldots, \ell)\right. \\
& +\sum_{\ell=3}^{n} \frac{\langle\ell h\rangle}{\langle\ell|1| h\rangle} c(2,3, \ldots, \ell)+\sum_{\ell=3}^{n} \frac{\langle\ell h\rangle}{\langle\ell|1| h\rangle} c(2,3, \ldots, \ell, 1) \\
& \left.+\sum_{\ell=3}^{n} \frac{\langle h \ell+1\rangle}{\langle h|1| \ell+1\rangle} c(2,3, \ldots, \ell, 1)\right] .
\end{aligned}
$$

We may shift the summation on the last term in (3.55) by 1 , so that $\ell$ runs from 4 to $n+1$. This causes the same factor $\frac{\langle h \ell\rangle}{\langle h|1| \ell\rangle}$ to appear here as in the other sums. The $\ell=n+1$ term may be discarded since $\langle h h\rangle=0$. The first term in (3.55) is exactly what is required to restore $\ell=3$ to the shifted sum. Hence, the final result is

$$
\mathcal{X}\left(1,2^{*}, 3, \ldots, n\right)=\frac{-k_{2}^{2}}{\langle h|3, \ldots, n| h\rangle} \sum_{\ell=3}^{n} \frac{\langle\ell h\rangle}{\langle\ell|1| h\rangle} c_{2}(1,2, \ldots, \ell),
$$

where

$$
\begin{aligned}
c_{2}(1,2, \ldots, \ell) \equiv & c(1,2, \ldots, \ell)-c(2,3, \ldots, \ell) \\
& -c(\ell, \ell-1, \ldots, 2,1)+c(\ell-1, \ell-2, \ldots, 2,1) .
\end{aligned}
$$

The coefficient $c_{2}(1,2, \ldots, \ell)$ is fully symmetric under permutation of all but its first and last arguments. It is immediately obvious from the definition (3.57) plus the fact that $c(1,2, \ldots, \ell)$ is symmetric in its first $\ell-1$ arguments that

$$
c_{2}(1,2, \ldots, \ell)=-c_{2}(\ell, \ell-1, \ldots, 1)
$$

Next, we compute $\mathcal{X}\left(1,2,3^{*}, 4, \ldots, n\right)$ by using the sum rule $(3.6 \mathrm{c})$, written in the form

$$
\begin{aligned}
\mathcal{X}_{12} & \equiv-\mathcal{X}\left(1,2,3^{*}, 4, \ldots, n\right)-\mathcal{X}\left(2,1,3^{*}, 4, \ldots, n\right) \\
& =\mathcal{X}\left(2,3^{*}, 1,4, \ldots, n\right)+\sum_{i=4}^{n} \mathcal{X}\left(2,3^{*}, 4, \ldots, i, 1, i+1, \ldots, n\right) .
\end{aligned}
$$


After performing a series of steps similar in spirit to those producing equations (3.49)-(3.56), we obtain

$$
\begin{aligned}
\mathcal{X}_{12}=\frac{k_{3}^{2}}{\langle h|4, \ldots, n| h\rangle} & \sum_{\ell=4}^{n} \frac{\langle\ell h\rangle}{\langle\ell|1| h\rangle}\left[\frac{\langle\ell h\rangle}{\langle\ell|2| h\rangle} c_{2}(2,1,3,4, \ldots, \ell)\right. \\
& -\frac{\langle\ell h\rangle}{\langle\ell|2| h\rangle} c_{2}(2,3, \ldots, \ell)-\frac{\langle 1 h\rangle}{\langle 1|2| h\rangle} c_{2}(2,3, \ldots, \ell, 1) \\
& \left.+\frac{\langle 1 h\rangle}{\langle 1|2| h\rangle} c_{2}(2,3, \ldots, \ell-1,1)\right]
\end{aligned}
$$

The terms in the square brackets may be simplified. Denote these terms by $\Xi$. Using the definition (3.57) we have

$$
\begin{aligned}
\Xi=\frac{\langle\ell h\rangle}{\langle\ell|2| h\rangle}\{ & c(2,1,3,4, \ldots, \ell)-c(1,3,4, \ldots, \ell) \\
& -c(1,3,4, \ldots, \ell, 2)+c(1,3,4, \ldots, \ell-1,2) \\
& -c(2,3, \ldots, \ell)+c(3,4, \ldots, \ell) \\
& +c(3,4, \ldots, \ell, 2)-c(3,4, \ldots, \ell-1,2)\} \\
-\frac{\langle 1 h\rangle}{\langle 1|2| h\rangle} & \{c(2,3, \ldots, \ell, 1)-c(3,4, \ldots, \ell, 1) \\
& -c(3,4, \ldots, \ell, 1,2)+c(3,4, \ldots, \ell, 2) \\
& -c(2,3, \ldots, \ell-1,1)+c(3,4, \ldots, \ell-1,1) \\
& +c(3,4, \ldots, \ell-1,1,2)-c(3,4, \ldots, \ell-1,2)\} .
\end{aligned}
$$

Judicious use of (A.15) and the symmetry of the $c$ 's allows us to rewrite this as

$$
\begin{aligned}
\Xi=\frac{\langle\ell h\rangle}{\langle\ell|2| h\rangle}\{c(1,2, \ldots, \ell)-c(1,3,4, \ldots, \ell) \\
-c(2,3, \ldots, \ell)+c(3,4, \ldots, \ell)\} \\
+\frac{\langle\ell 1\rangle}{\langle\ell|2| 1\rangle}\{c(3,4, \ldots, \ell, 2)-c(3,4, \ldots, \ell-1,2) \\
-c(1,3,4, \ldots, \ell, 2)+c(1,3,4, \ldots, \ell-1,2)\} \\
-\frac{\langle 1 h\rangle}{\langle 1|2| h\rangle}\{c(2,3, \ldots, \ell, 1)-c(3,4, \ldots, \ell, 1) \\
-c(2,3, \ldots, \ell-1,1)+c(3,4, \ldots, \ell-1,1)\} .
\end{aligned}
$$


Inserting (3.62) back into (3.60) produces

$$
\begin{gathered}
\mathcal{X}_{12}=\frac{k_{3}^{2}}{\langle h|4, \ldots, n| h\rangle} \sum_{\ell=4}^{n}\left[\frac{\langle\ell h\rangle^{2}}{\langle\ell|1| h\rangle\langle\ell|2| h\rangle}\{c(1,2, \ldots, \ell)-c(1,3,4, \ldots, \ell)\right. \\
-c(2,3, \ldots, \ell)+c(3,4, \ldots, \ell)\} \\
+\frac{\langle\ell h\rangle}{\langle\ell|2,1| h\rangle}\{c(3,4, \ldots, \ell, 2)-c(3,4, \ldots, \ell-1,2) \\
\quad-c(1,3,4, \ldots, \ell, 2)+c(1,3,4, \ldots, \ell-1,2)\} \\
-\frac{\langle\ell h\rangle}{\langle\ell|1,2| h\rangle}\{c(2,3, \ldots, \ell, 1)-c(3,4, \ldots, \ell, 1) \\
-c(2,3, \ldots, \ell-1,1)+c(3,4, \ldots, \ell-1,1)\} .
\end{gathered}
$$

Finally, note that we may use (A.15) to show that

$$
\frac{\langle\ell h\rangle^{2}}{\langle\ell|1| h\rangle\langle\ell|2| h\rangle}=\frac{\langle\ell h\rangle}{\langle\ell|1,2| h\rangle}+\frac{\langle\ell h\rangle}{\langle\ell|2,1| h\rangle} .
$$

Recalling that

$$
\mathcal{X}_{12}=-\mathcal{X}\left(1,2,3^{*}, 4, \ldots, n\right)-\mathcal{X}\left(2,1,3^{*}, 4, \ldots, n\right),
$$

we must disentangle the two contributions present in (3.63). This is accomplished by considering the $n=4$ case explicitly, for which we are able to obtain an expression for $\mathcal{X}\left(1,2,3^{*}, 4\right)$ from the relation ( $c f$. equation $(3.6 \mathrm{a})$ )

$$
\mathcal{X}\left(1,2,3^{*}, 4\right)=(-1)^{3} \mathcal{X}\left(4,3^{*}, 2,1\right)
$$

The result of this process is

$$
\begin{aligned}
\mathcal{X}\left(1,2,3^{*}, 4, \ldots, n\right)=\frac{-k_{3}^{2}}{\langle h|4, \ldots, n| h\rangle} \sum_{\ell=4}^{n} & {\left[\frac{\langle\ell h\rangle}{\langle\ell|1,2| h\rangle} c_{2}(1,2, \ldots, \ell)\right.} \\
& \left.-\frac{\langle\ell h\rangle}{\langle\ell|2,1| h\rangle} c_{2}(2,3, \ldots, \ell)\right] .
\end{aligned}
$$

We may write (3.67) in the suggestive form

$$
\begin{aligned}
& \mathcal{X}\left(1,2,3^{*}, 4, \ldots, n\right)= \\
& \quad=\frac{k_{3}^{2}}{\langle h|1,2| h\rangle\langle h|4,5, \ldots, n| h\rangle} \sum_{j=1}^{2} \sum_{\ell=4}^{n} \frac{\langle j|h| \ell\rangle}{\langle j \ell\rangle} c_{2}(j, \ldots, \ell) .
\end{aligned}
$$


Likewise, (3.56) may be recast as

$$
\begin{aligned}
& \mathcal{X}\left(1,2^{*}, 3, \ldots, n\right)= \\
& \quad=\frac{k_{2}^{2}}{\langle h|1| h\rangle\langle h|3,4, \ldots, n| h\rangle} \sum_{j=1}^{1} \sum_{\ell=4}^{n} \frac{\langle j|h| \ell\rangle}{\langle j \ell\rangle} c_{2}(j, \ldots, \ell) .
\end{aligned}
$$

Equations (3.68) and (3.69) lead us to the ansatz

$$
\begin{aligned}
& \mathcal{X}\left(1, \ldots, m^{*}, \ldots, n\right)= \\
& \quad=\frac{k_{m}^{2}}{\langle h|1, \ldots, m-1| h\rangle\langle h|m+1, \ldots, n| h\rangle} \sum_{j=1}^{m-1} \sum_{\ell=m+1}^{n} \frac{\langle j|h| \ell\rangle}{\langle j \ell\rangle} c_{2}(j, \ldots, \ell),
\end{aligned}
$$

valid for $2 \leq m \leq n-1$.

In order to prove that $(3.70)$ is correct, we must return to the recursion relation $(2.16)$, which becomes

$$
\begin{aligned}
& \mathcal{I}_{\alpha \dot{\alpha}}\left(1^{+}, \ldots, m^{*}, \ldots, n^{+}\right)= \\
& =\frac{-\sqrt{2}}{\kappa^{2}(1, n)} \sum_{i=1}^{m-1} \mathcal{J}_{\alpha \dot{\beta}}\left(1^{+}, \ldots, i^{+}\right) \overline{\mathcal{I}}^{\dot{\beta} \beta}\left((i+1)^{+}, \ldots, m^{*}, \ldots, n^{+}\right) \kappa_{\beta \dot{\alpha}}(1, n) \\
& +\frac{-\sqrt{2}}{\kappa^{2}(1, n)} \sum_{i=m}^{n-1} \mathcal{I}_{\alpha \dot{\beta}}\left(1^{+}, \ldots, m^{*}, \ldots, i^{+}\right) \overline{\mathcal{J}}^{\dot{\beta} \beta}\left((i+1)^{+}, \ldots, n^{+}\right) \kappa_{\beta \dot{\alpha}}(1, n) .
\end{aligned}
$$

Using (3.46) to replace $\mathcal{I}$ by $\mathcal{X}$ and (2.17) for the factors of $\mathcal{J}$ appearing in (3.71) yields

$$
\begin{aligned}
& \mathcal{X}\left(1, \ldots, m^{*}, \ldots, n\right)= \\
& =\frac{-1}{\kappa^{2}(1, n)}\left\{\sum_{i=1}^{m-1} \frac{u^{\gamma}(h) \kappa_{\gamma \dot{\gamma}}(1, i) \bar{\kappa}^{\dot{\gamma} \delta}(i+1, n) u_{\delta(h)}}{\langle h|1, \ldots, i| h\rangle} \mathcal{X}\left(i+1, \ldots, m^{*}, \ldots, n\right)\right. \\
& \left.\quad+\sum_{i=m}^{n-1} \frac{u^{\gamma}(h) \kappa_{\gamma \dot{\gamma}}(1, i) \bar{\kappa}^{\dot{\gamma} \delta}(i+1, n) u_{\delta(h)}}{\langle h|i+1, \ldots, n| h\rangle} \mathcal{X}\left(1, \ldots, m^{*}, \ldots, i\right)\right\}
\end{aligned}
$$

after a bit of algebra. We should like to prove that the ansatz (3.70) is correct by induction. Note that we already know the correct form for $\mathcal{X}\left(1^{*}, 2, \ldots, n\right)$ (equation (3.47); we may use that in combination with (3.6a) to obtain $\mathcal{X}\left(1,2, \ldots, n^{*}\right)$ :

$$
\mathcal{X}\left(1,2, \ldots, n^{*}\right)=\frac{k_{n}^{2}}{\langle h|1, \ldots, n-1| h\rangle} \sum_{j=1}^{n-1} c(n, n-1, \ldots, j) .
$$


Since the form of the solution is different when $m^{*}$ appears first or last in the argument list, we must handle those terms separately. So, replacing the $\mathcal{X}$ 's on the right hand side of $(3.72)$ with their values produces

$$
\begin{aligned}
& \mathcal{X}\left(1, \ldots, m^{*}, \ldots, n\right)=\frac{-k_{m}^{2}}{\kappa^{2}(1, n)} \frac{1}{\langle h|1, \ldots, m-1| h\rangle\langle h|m+1, \ldots, n| h\rangle} \\
& \times\left\{\sum_{i=m+1}^{n-1} \sum_{r=1}^{m-1} \sum_{s=m+1}^{i} u^{\gamma}(h) \kappa_{\gamma \dot{\gamma}}(1, n) \bar{\kappa}^{\dot{\gamma} \delta}(i+1, n) u_{\delta}(h) \frac{\langle i i+1\rangle}{\langle i|h| i+1\rangle} \frac{\langle r|h| s\rangle}{\langle r s\rangle} c_{2}(r, \ldots, s)\right. \\
& \quad+u^{\gamma}(h) \kappa_{\gamma \dot{\gamma}}(1, m) \bar{\kappa}^{\dot{\gamma} \delta}(m+1, n) u_{\delta}(h) \sum_{r=1}^{m-1} c(m, m-1, \ldots, r) \\
& \quad-u^{\gamma}(h) \kappa_{\gamma \dot{\gamma}}(1, m-1) \bar{\kappa}^{\dot{\gamma} \delta}(m, n) u_{\delta}(h) \sum_{s=m+1}^{n} c(m, m+1, \ldots, s) \\
& \left.\quad+\sum_{i=1}^{m-2} \sum_{r=i+1}^{m-1} \sum_{s=m+1}^{n} u^{\gamma}(h) \kappa_{\gamma} \dot{\gamma}(1, i) \bar{\kappa}^{\dot{\gamma} \delta}(1, n) u_{\delta}(h) \frac{\langle i i+1\rangle}{\langle i|h| i+1\rangle} \frac{\langle r|h| s\rangle}{\langle r s\rangle} c_{2}(r, \ldots, s)\right\} .
\end{aligned}
$$

We have used (A.6) and (A.13a) to write

$$
\begin{aligned}
u^{\gamma}(h) \kappa_{\gamma \dot{\gamma}}(1, i) \bar{\kappa}^{\dot{\gamma} \delta}(i+1, n) u_{\delta}(h) & =u^{\gamma}(h) \kappa_{\gamma \dot{\gamma}}(1, n) \bar{\kappa}^{\dot{\gamma} \delta}(i+1, n) u_{\delta}(h) \\
& =u^{\gamma}(h) \kappa_{\gamma \dot{\gamma}}(1, i) \bar{\kappa}^{\dot{\gamma} \delta}(1, n) u_{\delta}(h)
\end{aligned}
$$

where the first line is used in the first term of (3.74) and the second line in the fourth term.

We begin to simplify (3.74) by evaluating the sums on $i$ appearing in the first and fourth terms. Consider the first term $\left(\equiv \mathcal{X}_{1}\right)$ in the curly brackets of (3.74). Writing out the $i$-dependent $\kappa$-sum as a sum on $j$ we have

$$
\begin{aligned}
\mathcal{X}_{1} & =\sum_{i=m+1}^{n-1} \sum_{j=i+1}^{n} \sum_{r=1}^{m-1} \sum_{s=m+1}^{i} u^{\gamma}(h) \kappa_{\gamma}(1, n) k_{j}^{\dot{\gamma} \delta} u_{\delta}(h) \frac{\langle i i+1\rangle}{\langle i|h| i+1\rangle} \frac{\langle r|h| s\rangle}{\langle r s\rangle} c_{2}(r, \ldots, s) \\
& =\sum_{r=1}^{m-1} \sum_{s=m+1}^{n-1} \sum_{j=s+1}^{n} \sum_{i=s}^{j-1} u^{\gamma}(h) \kappa_{\gamma} \dot{\gamma}(1, n) \bar{u}^{\dot{\gamma}}\left(k_{j}\right)\langle j h\rangle \frac{\langle i i+1\rangle}{\langle i|h| i+1\rangle} \frac{\langle r|h| s\rangle}{\langle r s\rangle} c_{2}(r, \ldots, s) .
\end{aligned}
$$


Using (A.16) to perform the sum on $i$ yields

$$
\begin{aligned}
\mathcal{X}_{1} & =\sum_{r=1}^{m-1} \sum_{s=m+1}^{n-1} \sum_{j=s+1}^{n} u^{\gamma}(h) \kappa_{\gamma \dot{\gamma}}(1, n) \bar{u}^{\dot{\gamma}}\left(k_{j}\right)\langle j h\rangle \frac{\langle s j\rangle}{\langle s|h| j\rangle} \frac{\langle r|h| s\rangle}{\langle r s\rangle} c_{2}(r, \ldots, s) \\
& =\sum_{r=1}^{m-1} \sum_{s=m+1}^{n-1} u^{\gamma}(h) \kappa_{\gamma} \dot{\gamma}(1, n) \bar{\kappa}^{\dot{\gamma} \delta}(s+1, n) u_{\delta}\left(k_{s}\right) \frac{\langle h r\rangle}{\langle r s\rangle} c_{2}(r, \ldots, s),
\end{aligned}
$$

where in the second line we have cancelled common factors and restored the implicit $\kappa$-sum from the explicit sum on $j$. Employing the same sequence of steps on the fourth term in the curly brackets of (3.74) results in

$$
\mathcal{X}_{4}=\sum_{r=2}^{m-1} \sum_{s=m+1}^{n} u^{\gamma}\left(k_{r}\right) \kappa_{\gamma \dot{\gamma}}(1, r-1) \bar{\kappa}^{\dot{\gamma} \delta}(1, n) u_{\delta}(h) \frac{\langle s h\rangle}{\langle r s\rangle} c_{2}(r, \ldots, s)
$$

Combining (3.77) and (3.78) with (3.74) produces

$$
\begin{aligned}
\mathcal{X}\left(1, \ldots, m^{*}, \ldots, n\right)=\frac{-k_{m}^{2}}{\kappa^{2}(1, n)} \frac{1}{\langle h|1, \ldots, m-1| h\rangle\langle h|m+1, \ldots, n| h\rangle} \\
\times\left\{\sum_{r=1}^{m-1} \sum_{s=m+1}^{n-1} u^{\gamma}(h) \kappa_{\gamma \dot{\gamma}}(1, n) \bar{\kappa}^{\dot{\gamma} \delta}(s+1, n) u_{\delta}\left(k_{s}\right) \frac{\langle h r\rangle}{\langle r s\rangle} c_{2}(r, \ldots, s)\right. \\
\quad+u^{\gamma}(h) \kappa_{\gamma}(1, m) \bar{\kappa}^{\dot{\gamma} \delta}(m+1, n) u_{\delta}(h) \sum_{r=1}^{m-1} c(m, m-1, \ldots, r) \\
\quad-u^{\gamma}(h) \kappa_{\gamma \dot{\gamma}}(1, m-1) \bar{\kappa}^{\dot{\gamma} \delta}(m, n) u_{\delta}(h) \sum_{s=m+1}^{n} c(m, m+1, \ldots, s) \\
\left.\quad+\sum_{r=2}^{m-1} \sum_{s=m+1}^{n} u^{\gamma}\left(k_{r}\right) \kappa_{\gamma} \dot{\gamma}(1, r-1) \bar{\kappa}^{\dot{\gamma} \delta}(1, n) u_{\delta}(h) \frac{\langle s h\rangle}{\langle r s\rangle} c_{2}(r, \ldots, s)\right\} .
\end{aligned}
$$

We now write $\kappa(s+1, n)$ as $\kappa(1, n)-\kappa(1, s)$ and $\kappa(1, r-1)$ as $\kappa(1, n)-\kappa(s, n)$ in order to extract explicit factors of $\kappa^{2}(1, n)$ where possible. Rearranging slightly, 
we obtain

$$
\begin{aligned}
\mathcal{X}\left(1, \ldots, m^{*}, \ldots, n\right)=\frac{-k_{m}^{2}}{\kappa^{2}(1, n)} \frac{1}{\langle h|1, \ldots, m-1| h\rangle\langle h|m+1, \ldots, n| h\rangle} \\
\times\left\{-\kappa(1, n)^{2} \sum_{r=1}^{m-1} \sum_{s=m+1}^{n-1} \frac{\langle r|h| s\rangle}{\langle r s\rangle} c_{2}(r, \ldots, s)\right. \\
\quad-\kappa(1, n)^{2} \sum_{r=2}^{m-1} \sum_{s=m+1}^{n} \frac{\langle r|h| s\rangle}{\langle r s\rangle} c_{2}(r, \ldots, s) \\
\quad-\sum_{r=1}^{m-1} \sum_{s=m+1}^{n-1} u^{\gamma}(h) \kappa_{\gamma} \dot{\gamma}(1, n) \bar{\kappa}^{\dot{\gamma} \delta}(1, s) u_{\delta}\left(k_{s}\right) \frac{\langle h r\rangle}{\langle r s\rangle} c_{2}(r, \ldots, s) \\
\quad-\sum_{r=2}^{m-1} \sum_{s=m+1}^{n} u^{\gamma}\left(k_{r}\right) \kappa_{\gamma}(r, n) \bar{\kappa}^{\dot{\gamma} \delta}(1, n) u_{\delta}(h) \frac{\langle s h\rangle}{\langle r s\rangle} c_{2}(r, \ldots, s) \\
+u^{\gamma}(h) \kappa_{\gamma \dot{\gamma}}(1, m) \bar{\kappa}^{\dot{\gamma} \delta}(m+1, n) u_{\delta}(h) \sum_{r=1}^{m-1} c(m, m-1, \ldots, r) \\
\left.\quad-u^{\gamma}(h) \kappa_{\gamma \dot{\gamma}}(1, m-1) \bar{\kappa}^{\dot{\gamma} \delta}(m, n) u_{\delta}(h) \sum_{s=m+1}^{n} c(m, m+1, \ldots, s)\right\} .
\end{aligned}
$$

We may extend the limits of the first two sums from $r=1$ to $m-1$ and $s=m+1$ to $n$ by adding and subtracting the appropriate terms. The extra terms which arise are precisely the terms needed to extend the second pair of double sums in the same manner. Thus, all four double sums may be combined to give

$$
\begin{gathered}
\mathcal{X}\left(1, \ldots, m^{*}, \ldots, n\right)=\frac{-k_{m}^{2}}{\kappa^{2}(1, n)} \frac{1}{\langle h|1, \ldots, m-1| h\rangle\langle h|m+1, \ldots, n| h\rangle} \\
\times\left\{-\sum_{r=1}^{m-1} \sum_{s=m+1}^{n}\left[2 \kappa^{2}(1, n) \frac{\langle r|h| s\rangle}{\langle r s\rangle}\right.\right. \\
+u^{\gamma}(h) \kappa_{\gamma \dot{\gamma}}(1, n) \bar{\kappa}^{\dot{\gamma} \delta}(1, s) u_{\delta}\left(k_{s}\right) \frac{\langle h r\rangle}{\langle r s\rangle} \\
\left.+u^{\gamma}\left(k_{r}\right) \kappa_{\gamma}(r, n) \bar{\kappa}^{\dot{\gamma} \delta}(1, n) u_{\delta}(h) \frac{\langle s h\rangle}{\langle r s\rangle}\right] c_{2}(r, \ldots, s) \\
+u^{\gamma}(h) \kappa_{\gamma \dot{\gamma}}(1, m) \bar{\kappa}^{\dot{\gamma} \delta}(m+1, n) u_{\delta}(h) \sum_{r=1}^{m-1} c(m, m-1, \ldots, r) \\
\left.-u^{\gamma}(h) \kappa_{\gamma \dot{\gamma}}(1, m-1) \bar{\kappa}^{\dot{\gamma} \delta}(m, n) u_{\delta}(h) \sum_{s=m+1}^{n} c(m, m+1, \ldots, s)\right\} .
\end{gathered}
$$


Let us denote the argument of the double sum by $\Delta$ :

$$
\begin{gathered}
\Delta \equiv\left[2 \kappa^{2}(1, n) \frac{\langle r|h| s\rangle}{\langle r s\rangle}+u^{\gamma}(h) \kappa_{\gamma \dot{\gamma}}(1, n) \bar{\kappa}^{\dot{\gamma} \delta}(1, s) u_{\delta}\left(k_{s}\right) \frac{\langle h r\rangle}{\langle r s\rangle}\right. \\
\left.+u^{\gamma}\left(k_{r}\right) \kappa_{\gamma \dot{\gamma}}(r, n) \bar{\kappa}^{\dot{\gamma} \delta}(1, n) u_{\delta}(h) \frac{\langle s h\rangle}{\langle r s\rangle}\right] c_{2}(r, \ldots, s)
\end{gathered}
$$

In the second term of $\Delta$, we write $\kappa(1, s)$ as $\kappa(1, n)-\kappa(s+1, n)$. This gives

$$
\begin{gathered}
\Delta=\left[\kappa^{2}(1, n) \frac{\langle r|h| s\rangle}{\langle r s\rangle}-u^{\gamma}(h) \kappa_{\gamma \dot{\gamma}}(1, n) \bar{\kappa}^{\dot{\gamma} \delta}(s+1, n) u_{\delta}\left(k_{s}\right) \frac{\langle h r\rangle}{\langle r s\rangle}\right. \\
\left.+u^{\gamma}\left(k_{r}\right) \kappa_{\gamma \dot{\gamma}}(r, n) \bar{\kappa}^{\dot{\gamma} \delta}(1, n) u_{\delta}(h) \frac{\langle s h\rangle}{\langle r s\rangle}\right] c_{2}(r, \ldots, s)
\end{gathered}
$$

Using (A.8) to write

$$
u_{\delta}\left(k_{s}\right)\langle h r\rangle=-u_{\delta}(h)\langle r s\rangle-u_{\delta}\left(k_{r}\right)\langle s h\rangle
$$

in the second term of (3.83) produces

$$
\begin{aligned}
\Delta= & \kappa^{2}(1, n) \frac{\langle r|h| s\rangle}{\langle r s\rangle}+u^{\gamma}(h) \kappa_{\gamma \dot{\gamma}}(1, n) \bar{\kappa}^{\dot{\gamma} \delta}(s+1, n) u_{\delta}(h) \\
& +u^{\gamma}(h) \kappa_{\gamma \dot{\gamma}}(1, n) \bar{\kappa}^{\dot{\gamma} \delta}(s+1, n) u_{\delta}\left(k_{r}\right) \frac{\langle s h\rangle}{\langle r s\rangle} \\
& \left.+u^{\gamma}\left(k_{r}\right) \kappa_{\gamma \dot{\gamma}}(r, n) \bar{\kappa}^{\dot{\gamma} \delta}(1, n) u_{\delta}(h) \frac{\langle s h\rangle}{\langle r s\rangle}\right] c_{2}(r, \ldots, s) \\
= & {\left[\kappa^{2}(1, n) \frac{\langle r|h| s\rangle}{\langle r s\rangle}+u^{\gamma}(h) \kappa_{\gamma \dot{\gamma}}(1, n) \bar{\kappa}^{\dot{\gamma} \delta}(s+1, n) u_{\delta}(h)\right.} \\
& \left.+u^{\gamma}\left(k_{r}\right) \kappa_{\gamma \dot{\gamma}}(r, s) \bar{\kappa}^{\dot{\gamma} \delta}(1, n) u_{\delta}(h) \frac{\langle s h\rangle}{\langle r s\rangle}\right] c_{2}(r, \ldots, s) .
\end{aligned}
$$

We have used the antisymmetry of the spinor product to accomplish the matrix transposition required to produce the last line of (3.85).

Let us examine the third term $\left(\equiv_{3}\right)$ in $(3.85)$. Using (3.57) to write out the factor of $c_{2}(r, \ldots, s)$ we have

$$
\begin{aligned}
\Delta_{3}= & u^{\gamma}\left(k_{r}\right) \kappa_{\gamma \dot{\gamma}}(r, s) \bar{\kappa}^{\dot{\gamma} \delta}(1, n) u_{\delta}(h) \frac{\langle s h\rangle}{\langle r s\rangle} \\
\times & {[c(r, r+1, \ldots, s)-c(r+1, r+2, \ldots, s)} \\
& \quad-c(s, s-1, \ldots, r+1, r)+c(s-1, s-2, \ldots, r+1, r)]
\end{aligned}
$$


Employing (A.8) to write

$$
u^{\gamma}\left(k_{r}\right)\langle s h\rangle=-u^{\gamma}(h)\langle r s\rangle+u^{\gamma}\left(k_{s}\right)\langle r h\rangle
$$

produces

$$
\begin{aligned}
\Delta_{3}= & {\left[u^{\gamma}\left(k_{s}\right) \kappa_{\gamma \dot{\gamma}}(r, s) \bar{\kappa}^{\dot{\gamma} \delta}(1, n) u_{\delta}(h) \frac{\langle r h\rangle}{\langle r s\rangle}-u^{\gamma}(h) \kappa_{\gamma \dot{\gamma}}(r, s) \bar{\kappa}^{\dot{\gamma} \delta}(1, n) u_{\delta}(h)\right] } \\
& \times[c(r, r+1, \ldots, s)-c(r+1, r+2, \ldots, s)] \\
+ & u^{\gamma}\left(k_{r}\right) \kappa_{\gamma \dot{\gamma}}(r, s) \bar{\kappa}^{\dot{\gamma} \delta}(1, n) u_{\delta}(h) \frac{\langle s h\rangle}{\langle r s\rangle} \\
& \times[c(s-1, s-2, \ldots, r)-c(s, s-1, \ldots, r)] .
\end{aligned}
$$

Inserting the definition (3.48) for some of the $c$-functions and rearranging a bit we arrive at

$$
\begin{aligned}
\Delta_{3}=-u^{\gamma}(h) \kappa_{\gamma \dot{\gamma}}(r, s) \bar{\kappa}^{\dot{\gamma} \delta}(1, n) u_{\delta}(h)[c(r, r+1, \ldots, s)-c(r+1, r+2, \ldots, s)] \\
+\frac{\langle r|h| s\rangle}{\langle r s\rangle}\left\{\frac{u^{\epsilon}(h) \kappa_{\epsilon \dot{\epsilon}}(r+1, s) \bar{k}_{s}^{\dot{\epsilon} \gamma} \kappa(r, s)_{\gamma \dot{\gamma}} \bar{\kappa}^{\dot{\gamma} \delta}(1, n) u_{\delta}(h)}{\kappa^{2}(r+1, s) \kappa^{2}(r+1, s-1)}\right. \\
-\frac{u^{\epsilon}(h) \kappa_{\epsilon \dot{\epsilon}}(r, s) \bar{k}_{s}^{\dot{\epsilon} \gamma} \kappa(r, s)_{\gamma \dot{\gamma}} \bar{\kappa}^{\dot{\gamma} \delta}(1, n) u_{\delta}(h)}{\kappa^{2}(r, s) \kappa^{2}(r, s-1)} \\
+\frac{u^{\epsilon}(h) \kappa_{\epsilon \dot{\epsilon}}(r, s) \bar{k}_{r}^{\dot{\epsilon} \gamma} \kappa(r, s)_{\gamma \dot{\gamma}} \bar{\kappa}^{\dot{\gamma} \delta}(1, n) u_{\delta}(h)}{\kappa^{2}(r, s) \kappa^{2}(r+1, s)} \\
\left.-\frac{u^{\epsilon}(h) \kappa_{\epsilon \dot{\epsilon}}(r, s-1) \bar{k}_{r}^{\dot{\epsilon} \gamma} \kappa(r, s)_{\gamma \dot{\gamma}} \bar{\kappa}^{\dot{\gamma} \delta}(1, n) u_{\delta}(h)}{\kappa^{2}(r, s-1) \kappa^{2}(r+1, s-1)}\right\} .
\end{aligned}
$$

The terms in the curly brackets may be simplified by noting that

$$
\begin{aligned}
\kappa_{\alpha \dot{\alpha}}(1, s) \bar{k}_{s}^{\dot{\alpha} \beta} \kappa_{\beta \dot{\beta}}(1, s) & =\kappa_{\alpha \dot{\alpha}}(1, s) \bar{k}_{s}^{\dot{\alpha} \beta} \kappa_{\beta \dot{\beta}}(1, s-1) \\
& =\kappa_{\alpha \dot{\alpha}}(1, s)[\bar{\kappa}(1, s)-\bar{\kappa}(1, s-1)]^{\dot{\alpha} \beta} \kappa_{\beta \dot{\beta}}(1, s-1) \\
& =\kappa^{2}(1, s) \kappa_{\alpha \dot{\beta}}(1, s-1)-\kappa^{2}(1, s-1) \kappa_{\alpha \dot{\beta}}(1, s) .
\end{aligned}
$$

Note that in order to use (3.90) in the first and last terms in the curly brackets of (3.89), we must extend one of the $\kappa$-sums and compensate. This relation is 
useful in cancelling many of the denominators. The result of applying (3.90) to all four terms in (3.89) is

$$
\begin{aligned}
\Delta_{3}= & -u^{\gamma}(h) \kappa_{\gamma \dot{\gamma}}(r, s) \bar{\kappa}^{\dot{\gamma} \delta}(1, n) u_{\delta}(h)[c(r, r+1, \ldots, s)-c(r+1, r+2, \ldots, s)] \\
+ & \frac{\langle r|h| s\rangle}{\langle r s\rangle}\left[\frac{u^{\epsilon}(h) \kappa_{\epsilon \dot{\epsilon}}(r+1, s) \bar{k}_{s}^{\dot{\epsilon} \gamma} k_{r \gamma \dot{\gamma}} \bar{\kappa}^{\dot{\gamma} \delta}(1, n) u_{\delta}(h)}{\kappa^{2}(r+1, s) \kappa^{2}(r+1, s-1)}\right. \\
& \left.-\frac{\left.u^{\epsilon}(h) \kappa_{\epsilon \dot{\epsilon}}(r, s-1) \bar{k}_{r}^{\dot{\epsilon} \gamma} k_{s \gamma \dot{\gamma}} \bar{\kappa}^{\dot{\gamma} \delta}(1, n) u_{\delta}(h)\right]}{\kappa^{2}(r, s-1) \kappa^{2}(r+1, s-1)}\right] \\
+ & \frac{\langle r|h| s\rangle}{\langle r s\rangle}\left\{\frac{u^{\epsilon}(h) \kappa_{\epsilon \dot{\gamma}}(r+1, s-1) \bar{\kappa}^{\dot{\gamma} \delta}(1, n) u_{\delta}(h)}{\kappa^{2}(r+1, s-1)}\right. \\
& -\frac{u^{\epsilon}(h) \kappa_{\epsilon \dot{\gamma}}(r+1, s) \bar{\kappa}^{\dot{\gamma} \delta}(1, n) u_{\delta}(h)}{\kappa^{2}(r+1, s)}-\frac{u^{\epsilon}(h) \kappa_{\epsilon \dot{\gamma}}(r, s-1) \bar{\kappa}^{\dot{\gamma} \delta}(1, n) u_{\delta}(h)}{\kappa^{2}(r, s-1)} \\
& +\frac{u^{\epsilon}(h) \kappa_{\epsilon \dot{\gamma}}(r, s) \bar{\kappa}^{\dot{\gamma} \delta}(1, n) u_{\delta}(h)}{\kappa^{2}(r, s)}+\frac{u^{\epsilon}(h) \kappa_{\epsilon \dot{\gamma}}(r+1, s) \bar{\kappa}^{\dot{\gamma} \delta}(1, n) u_{\delta}(h)}{\kappa^{2}(r+1, s)} \\
& -\frac{u^{\epsilon}(h) \kappa_{\epsilon \dot{\gamma}}(r, s) \bar{\kappa}^{\dot{\gamma} \delta}(1, n) u_{\delta}(h)}{\kappa^{2}(r, s)}-\frac{u^{\epsilon}(h) \kappa_{\epsilon \dot{\gamma}}(r+1, s-1) \bar{\kappa}^{\dot{\gamma} \delta}(1, n) u_{\delta}(h)}{\kappa^{2}(r+1, s-1)} \\
& \left.+\frac{u^{\epsilon}(h) \kappa_{\epsilon \dot{\gamma}}(r, s-1) \bar{\kappa}^{\dot{\gamma} \delta}(1, n) u_{\delta}(h)}{\kappa^{2}(r, s-1)}\right\} \cdot
\end{aligned}
$$

The terms in curly brackets cancel among themselves, leaving just

$$
\begin{aligned}
\Delta_{3}= & -u^{\gamma}(h) \kappa_{\gamma \dot{\gamma}}(r, s) \bar{\kappa}^{\dot{\gamma} \delta}(1, n) u_{\delta}(h)[c(r, r+1, \ldots, s)-c(r+1, r+2, \ldots, s)] \\
& -\frac{u^{\epsilon}(h) \kappa_{\epsilon \dot{\epsilon}}(r+1, s) \bar{k}_{s}^{\dot{\epsilon} \alpha} u_{\alpha}(h)}{\kappa^{2}(r+1, s) \kappa^{2}(r+1, s-1)} u^{\gamma}(h) k_{r \gamma \dot{\gamma}} \bar{\kappa}^{\dot{\gamma} \delta}(1, n) u_{\delta}(h) \\
& -\frac{u^{\epsilon}(h) \kappa_{\epsilon \dot{\epsilon}}(r, s-1) \bar{k}_{r}^{\dot{\epsilon} \alpha} u_{\alpha}(h)}{\kappa^{2}(r, s-1) \kappa^{2}(r+1, s-1)} u^{\gamma}(h) k_{s \gamma \dot{\gamma}} \bar{\kappa}^{\dot{\gamma} \delta}(1, n) u_{\delta}(h) \\
= & -u^{\gamma}(h) \kappa_{\gamma \dot{\gamma}}(r, s) \bar{\kappa}^{\dot{\gamma} \delta}(1, n) u_{\delta}(h)[c(r, r+1, \ldots, s)-c(r+1, r+2, \ldots, s)] \\
& -u^{\gamma}(h) k_{r \gamma \dot{\gamma}} \bar{\kappa}^{\dot{\gamma} \delta}(1, n) u_{\delta}(h) c(r+1, r+2, \ldots, s) \\
& -u^{\gamma}(h) k_{s \gamma \dot{\gamma}} \bar{\kappa}^{\dot{\gamma} \delta}(1, n) u_{\delta}(h) c(s-1, s-2, \ldots, r),
\end{aligned}
$$

where we have simplified the remaining terms to extract factors of $c$. 
Substitution of (3.92) back into (3.85) to recover $\Delta$, and then putting $\Delta$ back into (3.81) to obtain $\mathcal{X}$ once more gives us

$$
\begin{aligned}
& \mathcal{X}\left(1, \ldots, m^{*}, \ldots, n\right)=\frac{-k_{m}^{2}}{\kappa^{2}(1, n)} \frac{1}{\langle h|1, \ldots, m-1| h\rangle\langle h|m+1, \ldots, n| h\rangle} \\
& \times\left\{-\kappa^{2}(1, n) \sum_{r=1}^{m-1} \sum_{s=m+1}^{n} \frac{\langle r|h| s\rangle}{\langle r s\rangle} c_{2}(r, \ldots, s)\right. \\
& +u^{\gamma}(h) \kappa_{\gamma \dot{\gamma}}(1, m) \bar{\kappa}^{\dot{\gamma} \delta}(m+1, n) u_{\delta}(h) \sum_{r=1}^{m-1} c(m, m-1, \ldots, r) \\
& -u^{\gamma}(h) \kappa_{\gamma \dot{\gamma}}(1, m-1) \bar{\kappa}^{\dot{\gamma} \delta}(m, n) u_{\delta}(h) \sum_{s=m+1}^{n} c(m, m+1, \ldots, s) \\
& +\sum_{r=1}^{m-1} \sum_{s=m+1}^{n}\left[u^{\delta}(h) \kappa_{\delta \dot{\gamma}}(r, n) \bar{\kappa}^{\dot{\gamma} \gamma}(1, n) u_{\gamma}(h)\right. \\
& \times[c(r, r+1, \ldots, s)-c(r+1, r+2, \ldots, s)] \\
& +u^{\delta}(h) \kappa_{\delta \dot{\gamma}}(s+1, n) \bar{\kappa}^{\dot{\gamma} \gamma}(1, n) u_{\gamma}(h) \\
& \times[c(s-1, s-2, \ldots, r)-c(s, s-1, \ldots, r)] \\
& +u^{\gamma}(h) k_{r \gamma \dot{\gamma}} \bar{\kappa}^{\dot{\gamma} \delta}(1, n) u_{\delta}(h) c(r+1, r+2, \ldots, s) \\
& \left.\left.+u^{\gamma}(h) k_{s \gamma \dot{\gamma}} \bar{\kappa}^{\dot{\gamma} \gamma}(1, n) u_{\delta}(h) c(s-1, s-2, \ldots, r)\right]\right\} \text {. }
\end{aligned}
$$

In addition to regrouping terms, we have made us of the symmetry of the arguments of $c$ in obtaining (3.93). Consider the second double sum in (3.93), which we call $\delta$ :

$$
\begin{aligned}
\delta \equiv & \sum_{r=1}^{m-1} \sum_{s=m+1}^{n} \sum_{j=r}^{n} u^{\delta}(h) k_{j \delta \dot{\gamma}} \bar{\kappa}^{\dot{\gamma} \gamma}(1, n) u_{\gamma}(h) \\
& \times[c(r, r+1, \ldots, s)-c(r+1, r+2, \ldots, s)] \\
& +\sum_{r=1}^{m-1} \sum_{s=m+1}^{n} \sum_{j=s+1}^{n} u^{\delta}(h) k_{j \delta \dot{\gamma}} \bar{\kappa}^{\dot{\gamma} \gamma}(1, n) u_{\gamma}(h) \\
& \times[c(s-1, s-2, \ldots, r)-c(s, s-1, \ldots, r)] \\
& +\sum_{r=1}^{m-1} \sum_{s=m+1}^{n}\left[u^{\gamma}(h) k_{r \gamma \dot{\gamma}} \bar{\kappa}^{\dot{\gamma} \delta}(1, n) u_{\delta}(h) c(r+1, r+2, \ldots, s)\right. \\
& \left.+u^{\gamma}(h) k_{s \gamma \dot{\gamma}} \bar{\kappa}^{\dot{\gamma} \gamma}(1, n) u_{\delta}(h) c(s-1, s-2, \ldots, r)\right] .
\end{aligned}
$$


In (3.94) we have written out the two implicit $\kappa$-sums that do not cover the full range 1 to $n$. We now prepare to do the sum on $r$ in the first term of (3.94) and the sum on $s$ in the second term:

$$
\begin{aligned}
\delta= & \sum_{s=m+1}^{n} \sum_{j=1}^{m-1} \sum_{r=1}^{j} u^{\delta}(h) k_{j \delta \dot{\gamma}} \bar{\kappa}^{\dot{\gamma} \gamma}(1, n) u_{\gamma}(h) \\
& \times[c(r, r+1, \ldots, s)-c(r+1, r+2, \ldots, s)] \\
& +\sum_{s=m+1}^{n} \sum_{j=m}^{n} \sum_{r=1}^{m-1} u^{\delta}(h) k_{j \delta} \bar{\kappa}^{\dot{\gamma} \gamma}(1, n) u_{\gamma}(h) \\
& +\sum_{r=1}^{m-1} \sum_{j=m+2}^{n} \sum_{s=m+1}^{j-1} u^{\delta}(h) k_{j \delta \dot{\gamma}} \bar{\kappa}^{\dot{\gamma} \gamma}(1, n) u_{\gamma}(h) \\
& +\sum_{r=1}^{m-1} \sum_{s=m+1}^{n}[c(s-1, s+1, \ldots, s)-c(r+1, r+2, \ldots, s)] \\
& +u^{\gamma}(h) k_{r \gamma \dot{\gamma}} \bar{\kappa}^{\gamma} \delta(1, n) u_{\delta}(h) c(r+1, r+2, \ldots, s) \\
& \left.+u^{\gamma}(h) k_{s \gamma \dot{\gamma}} \bar{\kappa}^{\dot{\gamma} \gamma}(1, n) u_{\delta}(h) c(s-1, s-2, \ldots, r)\right]
\end{aligned}
$$

We are forced to break the first term into two pieces when doing the summation interchange. The indicated sums are easy to do: only the endpoints survive, leaving

$$
\begin{aligned}
\delta= & \sum_{s=m+1}^{n} \sum_{j=1}^{m-1} u^{\delta}(h) k_{j \delta \dot{\gamma}} \bar{\kappa}^{\dot{\gamma} \gamma}(1, n) u_{\gamma}(h)[c(1,2, \ldots, s)-c(j+1, j+2, \ldots, s)] \\
& +\sum_{s=m+1}^{n} \sum_{j=m}^{n} u^{\delta}(h) k_{j \delta \dot{\gamma}} \bar{\kappa}^{\dot{\gamma} \gamma}(1, n) u_{\gamma}(h)[c(1,2, \ldots, s)-c(m, m+1, \ldots, s)] \\
& +\sum_{r=1}^{m-1} \sum_{j=m+2}^{n} u^{\delta}(h) k_{j \delta \dot{\gamma}} \bar{\kappa}^{\dot{\gamma} \gamma}(1, n) u_{\gamma}(h) \\
& +\sum_{r=1}^{m-1} \sum_{s=m+1}^{n}\left[u^{\gamma}(h) k_{r \gamma} \dot{\gamma} \bar{\kappa}^{\dot{\gamma} \delta}(1, n) u_{\delta}(h) c(r+1, r+2, \ldots, s)\right. \\
& \left.+u^{\gamma}(h) k_{s \gamma} \dot{\gamma} \bar{\kappa}^{\dot{\gamma} \gamma}(1, n) u_{\delta}(h) c(s-1, s-2, \ldots, r)\right] .
\end{aligned}
$$


The terms containing $c(1,2, \ldots, s)$ may be summed on $j$ and combined to give

$$
\begin{aligned}
\sum_{s=m+1}^{n} u^{\delta}(h) & {[\kappa(1, m-1)+\kappa(m, n)]_{\delta \dot{\gamma}} \bar{\kappa}^{\dot{\gamma} \gamma}(1, n) u_{\gamma}(h) c(1,2, \ldots, s)=} \\
& =\kappa^{2}(1, n)\langle h h\rangle \sum_{s=m+1}^{n} c(1,2, \ldots, s) \\
& =0 .
\end{aligned}
$$

The remaining terms may be written as

$$
\begin{aligned}
\delta= & -\sum_{j=1}^{m-1} \sum_{s=m+1}^{n} u^{\delta}(h) k_{j \delta \dot{\gamma}} \bar{\kappa}^{\dot{\gamma} \gamma}(1, n) u_{\gamma}(h) c(j+1, j+2, \ldots, s) \\
& -\sum_{s=m+1}^{n} u^{\delta}(h) \kappa_{\delta \dot{\gamma}}(m, n) \bar{\kappa}^{\dot{\gamma} \gamma}(1, n) u_{\gamma}(h) c(m, m+1, \ldots, s) \\
& +\sum_{r=1}^{m-1} u^{\delta}(h) \kappa_{\delta \dot{\gamma}}(m+2, n) \bar{\kappa}^{\dot{\gamma} \gamma}(1, n) u_{\gamma}(h) c(m, m-1, \ldots, r) \\
& -\sum_{r=1}^{m-1} \sum_{j=m+2}^{n} u^{\delta}(h) k_{j \delta \dot{\gamma}} \bar{\kappa}^{\dot{\gamma} \gamma}(1, n) u_{\gamma}(h) c(j-1, j-2, \ldots, r) \\
& +\sum_{r=1}^{m-1} \sum_{s=m+1}^{n}\left[u^{\gamma}(h) k_{r \gamma \dot{\gamma}} \bar{\kappa}^{\dot{\gamma} \delta}(1, n) u_{\delta}(h) c(r+1, r+2, \ldots, s)\right. \\
& \left.+u^{\gamma}(h) k_{s \gamma \dot{\gamma}} \bar{\kappa}^{\dot{\gamma} \gamma}(1, n) u_{\delta}(h) c(s-1, s-2, \ldots, r)\right] .
\end{aligned}
$$

Everything except for the $s=m+1$ term of the second term in the square brackets of the final double sum is cancelled by the first two double sums in (3.98). This leaves only

$$
\begin{aligned}
\delta= & -\sum_{s=m+1}^{n} u^{\delta}(h) \kappa_{\delta \dot{\gamma}}(m, n) \bar{\kappa}^{\dot{\gamma} \gamma}(1, n) u_{\gamma}(h) c(m, m+1, \ldots, s) \\
& +\sum_{r=1}^{m-1} u^{\delta}(h) \kappa_{\delta \dot{\gamma}}(m+2, n) \bar{\kappa}^{\dot{\gamma} \gamma}(1, n) u_{\gamma}(h) c(m, m-1, \ldots, r) \\
& +\sum_{r=1}^{m-1} u^{\gamma}(h)\left(k_{m+1}\right)_{\gamma \dot{\gamma}} \bar{\kappa}^{\dot{\gamma} \gamma}(1, n) u_{\delta}(h) c(m, m-1, \ldots, r) .
\end{aligned}
$$

The third line of (3.99) may be combined with the second line by extending $\kappa(m+2, n)$ to $\kappa(m+1, n)$. We then transpose the order of the matrix multiplica- 
tion and use (A.6) to achieve

$$
\begin{aligned}
\delta= & \sum_{s=m+1}^{n} u^{\gamma}(h) \kappa_{\gamma \dot{\gamma}}(1, m-1) \bar{\kappa}^{\dot{\gamma} \delta}(m, n) u_{\delta}(h) c(m, m+1, \ldots, s) \\
& -\sum_{r=1}^{m-1} u^{\gamma}(h) \kappa_{\gamma \dot{\gamma}}(1, m) \bar{\kappa}^{\dot{\gamma} \delta}(m+1, n) u_{\delta}(h) c(m, m-1, \ldots, r) .
\end{aligned}
$$

A comparison of (3.100) with (3.93) shows that the contribution from $\delta$ exactly cancels the contribution from the two remaining single sums appearing in $\mathcal{X}$. Thus, we have

$$
\begin{aligned}
& \mathcal{X}\left(1, \ldots, m^{*}, \ldots, n\right)= \\
& \quad=\frac{k_{m}^{2}}{\langle h|1, \ldots, m-1,| h\rangle\langle h|m+1, \ldots, n| h\rangle} \sum_{r=1}^{m-1} \sum_{s=m+1}^{n} \frac{\langle r|h| s\rangle}{\langle r s\rangle} c_{2}(r, \ldots, s),
\end{aligned}
$$

proving the ansatz (3.70).

\section{LIKE-HELICITY GLUON PRODUCTION}

In this section we present the computation of the process (1.1) for the case where all of the gluons have the same helicity. We begin by examining the currents which must be sewn together to form the amplitude for (1.1). We will see that the modified gluon current discussed in the previous section arises in a natural manner within this context. After evaluating the color factor and the sub-leading contribution in $\frac{1}{N}$, we engage in a lengthy discussion of the main contribution. In spite of a very complicated starting point, we will end up with a relatively simple final result, in agreement with Mangano [6].

\subsection{Preliminary considerations}

Figure 1 illustrates the form of the tree-level Feynman graphs contributing to (1.1). In terms of the currents introduced in the second and third sections, we see that it consists of two quark currents, two antiquark currents, and 
a gluon current with two off-shell gluons. It is convenient to define a current $\left(\Gamma_{\xi}^{x}\right)_{j i}\left(p ; 1, \ldots, n ; p^{\prime}\right)$ consisting of a quark line, $n$ on-shell gluons, and one offshell gluon. With all momenta directed inward, this current has an antiquark of momentum $p$ and color index $j$, a quark of momentum $p^{\prime}$ and color index $i, n$ on-shell gluons with momenta $k_{\ell}$ and color indices $a_{\ell}$, and an off-shell gluon of momentum $-p-\kappa(1, n)-p^{\prime}$, Lorentz index $\xi$ and color index $x$. We do not include the propagator for the off-shell gluon in the definition of $\left(\Gamma_{\xi}^{x}\right)_{j i}$. According to this definition we have

$$
\begin{aligned}
& \left(\Gamma_{\xi}^{x}\right)_{j i}\left(p ; 1, \ldots, n ; p^{\prime}\right)= \\
& \quad=\sum_{\mathcal{P}(1 \ldots n)} \sum_{s=0}^{n} \frac{\widehat{\bar{\psi}}_{j k}(p ; 1, \ldots, s)}{s !}\left[-i g\left(T^{x}\right)_{k m} \gamma_{\xi}\right] \frac{\widehat{\psi}_{m i}\left(s+1, \ldots, n ; p^{\prime}\right)}{(n-s) !} .
\end{aligned}
$$

Insertion of the color factorizations (2.19a) and (2.22a) into (4.1) produces the form

$$
\begin{aligned}
&\left(\Gamma_{\xi}^{x}\right)_{j i}\left(p ; 1, \ldots, n ; p^{\prime}\right)= \\
&=-i g^{n+1} \sum_{\mathcal{P}(1 \ldots n)} \sum_{s=0}^{n}\left(\Omega[1, s] T^{x} \Omega[s+1, n]\right)_{j i} \\
& \quad \times \bar{\psi}(p ; 1, \ldots, s) \gamma_{\xi} \psi\left(s+1, \ldots, n ; p^{\prime}\right) .
\end{aligned}
$$

We now turn to the current with two off-shell gluons. From (2.7a) we know that

$$
\begin{aligned}
& \widehat{J}_{\xi}^{x}(0,1, \ldots, n)= \\
& \quad=2 g^{n} \sum_{\mathcal{P}(0 \ldots n)} \operatorname{tr}\left(\Omega[0, n] T^{x}\right) J_{\xi}(0,1, \ldots, n) \\
& \quad=2 g^{n} \sum_{\mathcal{P}(1 \ldots n)} \sum_{s=0}^{n} \operatorname{tr}\left(\Omega[1, s] T^{a_{0}} \Omega[s+1, n] T^{x}\right) J_{\xi}(1, \ldots, s, 0, s+1, \ldots, n) .
\end{aligned}
$$

To obtain a formal expression for the current with two off-shell gluons, we differentiate (4.3) with respect to the polarization vector of gluon number 0 and restore its propagator:

$$
\begin{aligned}
& \widehat{I}_{\xi \zeta}^{x z}\left(0^{*}, 1, \ldots, n\right)= \\
& \quad=2 g^{n} \frac{-i}{k_{0}^{2}} \sum_{\mathcal{P}(1 \ldots n)} \sum_{s=0}^{n} \operatorname{tr}\left(\Omega[1, s] T^{z} \Omega[s+1, n] T^{x}\right) \frac{\partial}{\partial \epsilon^{\zeta}(0)} J_{\xi}(1, \ldots, s, 0, s+1, \ldots, n) .
\end{aligned}
$$


Let us denote by $\mathcal{M}_{k \ell}^{i j}\left(p, q ; 1, \ldots, n ; p^{\prime}, q^{\prime}\right)$ the amplitude for the process represented by Figure 1. Putting the pieces together, we write

$$
\begin{aligned}
& \mathcal{M}_{k \ell}^{i j}\left(p, q ; 1, \ldots, n ; p^{\prime}, q^{\prime}\right) \equiv \\
&=\sum_{\mathcal{P}(1 \ldots n)} \sum_{v=0}^{n} \sum_{w=v}^{n} \frac{1}{v !} \widehat{I}_{\xi \zeta}^{x z}\left(0^{*}, 1, \ldots, v\right) \\
& \quad \times \frac{1}{(w-v) !}\left(\Gamma^{\xi x}\right)^{i j}\left(p ; v+1, \ldots, w ; p^{\prime}\right) \\
& \quad \times \frac{1}{(n-w) !}\left(\Gamma^{\zeta z}\right)_{k \ell}\left(q ; w+1, \ldots, n ; q^{\prime}\right)
\end{aligned}
$$

where momentum conservation tells us that

$$
\begin{aligned}
k_{0} & =q+\kappa(w+1, n)+q^{\prime} \\
& =-p-\kappa(1, w)-p^{\prime} .
\end{aligned}
$$

We are able to evaluate (4.5) in the case where all of the radiated gluons have the same helicity. Since the methods illustrated below handle all three of the possible quark-antiquark helicity combinations with no extra complications, we select the amplitude with two right-handed quark lines for further study. Clearly, this helicity combination will involve $\bar{\psi}^{\alpha}\left(q^{-} ;(w+1)^{+}, \ldots, s^{+}\right)$for various values of $s$, as implied by the form of (4.2). However, we know from (2.25) that this current may be made to vanish for $n \geq 1$ by the gauge choice $h=q$. Thus, the sum on $s$ in (4.2) may be replaced by a single term:

$$
\begin{aligned}
& \left(\Gamma^{\zeta z}\right)_{k \ell}\left(q^{-} ;(w+1)^{+}, \ldots, n^{+} ; q^{\prime+}\right)= \\
& \quad=-i g^{n-w+1} \sum_{\mathcal{P}(w+1 \ldots n)}\left(T^{z} \Omega[w+1, n]\right)_{k \ell} \bar{\psi}\left(q^{-}\right) \gamma^{\zeta} \psi\left((w+1)^{+}, \ldots, n^{+} ; q^{\prime+}\right) .
\end{aligned}
$$

Unfortunately, the same reduction is not permissible on the other quark line. Once $h=q$ is chosen for one of the currents, consistency forces us to choose $h=q$ in all of the other currents, since individual terms in the sum (4.5) are not gauge-invariant even though the sum as a whole is. 
Combination of (4.2), (4.4) and (4.7) with (4.5) produces

$$
\begin{aligned}
& \mathcal{M}_{k \ell}^{i j}\left(p^{-}, q^{-} ; 1^{+}, \ldots,\right.\left.n^{+} ; p^{+}, q^{\prime+}\right) \equiv \\
&=2 i g^{n+2} \sum_{\mathcal{P}(1 \ldots n)} \sum_{v=0}^{n} \sum_{w=v}^{n} \sum_{u=0}^{v} \sum_{t=v}^{w} \operatorname{tr}\left(\Omega[1, u] T^{z} \Omega[u+1, v] T^{x}\right) \\
& \quad \times\left(\Omega[v+1, t] T^{x} \Omega[t+1, w]\right)^{i j}\left(T^{z} \Omega[w+1, n]\right)_{k \ell} \\
& \quad \times \frac{1}{k_{0}^{2}} \frac{\partial}{\partial \epsilon^{\zeta}\left(0^{+}\right)} J_{\xi}\left(1^{+}, \ldots, u^{+}, 0^{+},(u+1)^{+}, \ldots, v^{+}\right) \\
& \times \bar{\psi}\left(p^{-} ;(v+1)^{+}, \ldots, t^{+}\right) \gamma^{\xi} \psi\left((t+1)^{+}, \ldots, w^{+} ; p^{\prime+}\right) \\
& \times \bar{\psi}\left(q^{-}\right) \gamma^{\zeta} \psi\left((w+1)^{+}, \ldots, n^{+} ; q^{\prime+}\right) .
\end{aligned}
$$

The multispinor replacement rules (A.22) tell us that this is equivalent to

$$
\begin{aligned}
& \mathcal{M}_{k \ell}^{i j}\left(p^{-}, q^{-} ; 1^{+}, \ldots, n^{+} ; p^{+}, q^{\prime+}\right)= \\
&=4 i g^{n+2} \sum_{\mathcal{P}(1 \ldots n)} \sum_{v=0}^{n} \sum_{w=v}^{n} \sum_{u=0}^{v} \sum_{t=v}^{w} \operatorname{tr}\left(\Omega[1, u] T^{z} \Omega[u+1, v] T^{x}\right) \\
& \times\left(\Omega[v+1, t] T^{x} \Omega[t+1, w]\right)^{i j}\left(T^{z} \Omega[w+1, n]\right)_{k \ell} \\
& \times \frac{1}{k_{0}^{2}} \frac{\partial}{\partial \bar{\epsilon}^{\dot{\beta} \beta}\left(0^{+}\right)} \mathcal{J}_{\alpha \dot{\alpha}}\left(1^{+}, \ldots, u^{+}, 0^{+},(u+1)^{+}, \ldots, v^{+}\right) \\
& \times \bar{\psi}^{\alpha}\left(p^{-} ;(v+1)^{+}, \ldots, t^{+}\right) \psi^{\dot{\alpha}}\left((t+1)^{+}, \ldots, w^{+} ; p^{\prime+}\right) \\
& \times \bar{\psi}^{\beta}\left(q^{-}\right) \psi^{\dot{\beta}}\left((w+1)^{+}, \ldots, n^{+} ; q^{\prime+}\right) .
\end{aligned}
$$

From $(2.17 \mathrm{~b}),(2.24 \mathrm{a}),(2.26)$, and $(2.27)$ we see that

$$
\begin{aligned}
\bar{\psi}^{\beta}\left(q^{-}\right) \psi^{\dot{\beta}}\left((w+1)^{+}, \ldots, n^{+} ; q^{\prime+}\right)= \\
=\frac{(-\sqrt{2})^{n-w} u^{\beta}(q)\left[\bar{\kappa}(w+1, n)+\bar{q}^{\prime}\right]^{\dot{\beta} \gamma} u_{\gamma}(q)}{\left\langle q|w+1, \ldots, n| q^{\prime}\right\rangle} .
\end{aligned}
$$

We may use the Weyl equation to add $\bar{q}^{\dot{\beta} \gamma}$ to the expression in square brackets in (4.10). But then we have

$$
\begin{aligned}
\bar{\psi}^{\beta}\left(q^{-}\right) \psi^{\dot{\beta}}\left((w+1)^{+}, \ldots, n^{+} ; q^{\prime+}\right) & =(-\sqrt{2})^{n-w} \frac{u^{\beta}(q) \bar{k}_{0}^{\dot{\beta} \gamma} u_{\gamma}(q)}{\left\langle q|w+1, \ldots, n| q^{\prime}\right\rangle} \\
& =(-\sqrt{2})^{n-w} \frac{-\overline{\mathcal{I}}^{\dot{\beta} \beta}\left(0^{*}\right)}{\left\langle q|w+1, \ldots, n| q^{\prime}\right\rangle},
\end{aligned}
$$

where $\overline{\mathcal{I}}^{\dot{\beta} \beta}\left(0^{*}\right)$ is precisely the type of "generalized" polarization spinor introduced in the last section ( $c f$. equation (3.7)). With this identification made, we 
see that equation (4.9) tells us to take the usual gluon current, remove the polarization spinor for the gluon labelled by "0", and replace it with a "generalized" polarization spinor, with $k_{0}^{2} \neq 0$. The result is exactly the modified gluon current discussed in the previous section. Hence,

$$
\begin{aligned}
& \mathcal{M}_{k \ell}^{i j}\left(p^{-}, q^{-} ; 1^{+}, \ldots\right.\left., n^{+} ; p^{+}, q^{+}\right)= \\
&=-4 i g^{n+2} \sum_{\mathcal{P}(1 \ldots n)} \sum_{v=0}^{n} \sum_{w=v}^{n} \sum_{u=0}^{v} \sum_{t=v}^{w} \operatorname{tr}\left(\Omega[1, u] T^{z} \Omega[u+1, v] T^{x}\right) \\
& \quad \times\left(\Omega[v+1, t] T^{x} \Omega[t+1, w]\right)^{i j}\left(T^{z} \Omega[w+1, n]\right)_{k \ell} \\
& \quad \times \frac{1}{k_{0}^{2}} \mathcal{I}_{\alpha \dot{\alpha}}\left(1^{+}, \ldots, u^{+}, 0^{*},(u+1)^{+}, \ldots, v^{+}\right) \\
& \quad \times \bar{\psi}^{\alpha}\left(p^{-} ;(v+1)^{+}, \ldots, t^{+}\right) \psi^{\dot{\alpha}}\left((t+1)^{+}, \ldots, w^{+} ; p^{+}\right) \\
& \quad \times(-\sqrt{2})^{n-w} \frac{1}{\left\langle q|w+1, \ldots, n| q^{\prime}\right\rangle}
\end{aligned}
$$

Inserting the appropriate expressions from section 2 for the remaining quark currents gives us

$$
\begin{aligned}
\mathcal{M}_{k \ell}^{i j}\left(p^{-}, q^{-} ; 1^{+}\right. & \left.\ldots, n^{+} ; p^{+}, q^{\prime+}\right)= \\
=-4 i g^{n+2} & \sum_{\mathcal{P}(1 \ldots n)} \sum_{v=0}^{n} \sum_{w=v}^{n} \sum_{u=0}^{v} \sum_{t=v}^{w} \operatorname{tr}\left(\Omega[1, u] T^{z} \Omega[u+1, v] T^{x}\right) \\
& \times\left(\Omega[v+1, t] T^{x} \Omega[t+1, w]\right)^{i j}\left(T^{z} \Omega[w+1, n]\right)_{k \ell} \\
& \times \frac{1}{k_{0}^{2}} \mathcal{I}_{\alpha \dot{\alpha}}\left(1^{+}, \ldots, u^{+}, 0^{*},(u+1)^{+}, \ldots, v^{+}\right) \\
& \times \frac{(-\sqrt{2})^{n-v}\langle p q\rangle u^{\alpha}(p)\left[\bar{\kappa}(t+1, w)+\bar{p}^{\prime}\right]^{\dot{\alpha} \beta} u_{\beta}(q)}{\langle p|v+1, \ldots, t| q\rangle\left\langle q|t+1, \ldots, w| p^{\prime}\right\rangle\left\langle q|w+1, \ldots, n| q^{\prime}\right\rangle} .
\end{aligned}
$$

\subsection{Evaluation of the color factor}

We turn our attention to the color factor in (4.13), which reads

$$
\begin{aligned}
& C_{k \ell}^{i j} \equiv \operatorname{tr}\left(\Omega[1, u] T^{z} \Omega[u+1, v] T^{x}\right)\left(\Omega[v+1, t] T^{x} \Omega[t+1, w]\right)^{i j}\left(T^{z} \Omega[w+1, n]\right)_{k \ell} \\
&=\left(\Omega[1, u] T^{z} \Omega[u+1, v]\right)^{p q}\left(T^{z} \Omega[w+1, n]\right)_{k \ell} \\
& \quad \times(\Omega[v+1, t])^{i r}(\Omega[t+1, w])^{s j}\left(T^{x}\right)^{q p}\left(T^{x}\right)^{r s} .
\end{aligned}
$$


In order to evaluate $C_{k \ell}^{i j}$, we only need to know the completeness relation for $S U(N)$, as given by (2.4). Evaluating the (implicit) sum on $x$ gives

$$
\begin{aligned}
C_{k \ell}^{i j}= & \frac{1}{2}\left(\Omega[v+1, t] \Omega[1, u] T^{z} \Omega[u+1, v] \Omega[t+1, w]\right)^{i j}\left(T^{z} \Omega[w+1, n]\right)_{k \ell} \\
& -\frac{1}{2 N} \operatorname{tr}\left(\Omega[1, u] T^{z} \Omega[u+1, v]\right)(\Omega[v+1, w])^{i j}\left(T^{z} \Omega[w+1, n]\right)_{k \ell} .
\end{aligned}
$$

The second term in (4.15) contains a trace. Since the trace is invariant under cyclic permutations, we may extract a factor

$$
\sum_{\mathcal{C}(0 \ldots v)} \mathcal{I}_{\alpha \dot{\alpha}}\left(1^{+}, \ldots, u^{+}, 0^{*},(u+1)^{+}, \ldots, v^{+}\right)=0, \quad v \neq 0
$$

from the permutation sum in (4.13). Equation (4.16), the form of (2.11b) satisfied by the modified current, tells us that there is no contribution to the amplitude from this term, except perhaps at $v=0$. When $v=0$, however, the trace contains just a single color matrix,

$$
\operatorname{tr} T^{z}=0
$$

Hence, the only non-vanishing contribution to (4.15) is generated by the first term and we may write

$$
\begin{aligned}
C_{k \ell}^{i j}= & \frac{1}{2}(\Omega[v+1, t] \Omega[1, u])^{i p}(\Omega[u+1, v] \Omega[t+1, w])^{q j} \\
& \times(\Omega[w+1, n])_{r \ell}\left(T^{z}\right)_{p q}\left(T^{z}\right)_{k r} \\
= & \frac{1}{4}(\Omega[v+1, t] \Omega[1, u] \Omega[w+1, n])_{\ell}^{i}(\Omega[u+1, v] \Omega[t+1, w])_{k}^{j} \\
& -\frac{1}{4 N}(\Omega[v+1, t] \Omega[1, v] \Omega[t+1, w])^{i j}(\Omega[w+1, n])_{k \ell},
\end{aligned}
$$

where we have performed the sum on $z$ to obtain the second line. Notice that the $\frac{1}{N}$ term in (4.18) is independent of $u$. Thus, the sum on $u$ appearing in (4.13) reads

$$
\sum_{u=0}^{v} \mathcal{I}_{\alpha \dot{\alpha}}\left(1^{+}, \ldots, u^{+}, 0^{*},(u+1)^{+}, \ldots, v^{+}\right)=0, \quad v \neq 0
$$


according to $(2.11 \mathrm{c})$. Thus, we see that the only term which gives a contribution has $v=0$, producing

$$
\begin{aligned}
C_{k \ell}^{i j}= & \frac{1}{4}(\Omega[v+1, t] \Omega[1, u] \Omega[w+1, n])_{\ell}^{i}(\Omega[u+1, v] \Omega[t+1, w])_{k}{ }^{j} \\
& -\frac{\delta_{v 0}}{4 N}(\Omega[1, w])^{i j}(\Omega[w+1, n])_{k \ell} .
\end{aligned}
$$

We now insert (4.20) into (4.13), yielding

$$
\begin{aligned}
\mathcal{M}_{k \ell}^{i j}\left(p^{-}, q^{-} ; 1^{+}, \ldots, n^{+} ; p^{+}, q^{+}\right)= & \\
=-i g^{n+2} & \sum_{\mathcal{P}(1 \ldots n)} \sum_{v=0}^{n} \sum_{w=v}^{n} \sum_{u=0}^{v} \sum_{t=v}^{w}(\Omega[v+1, t] \Omega[1, u] \Omega[w+1, n])^{i} \ell \\
& \times(\Omega[u+1, v] \Omega[t+1, w])_{k}^{j} \\
& \times \frac{1}{k_{0}^{2}} \mathcal{I}_{\alpha \dot{\alpha}}\left(1^{+}, \ldots, u^{+}, 0^{*},(u+1)^{+}, \ldots, v^{+}\right) \\
& \times \frac{(-\sqrt{2})^{n-v}\langle p q\rangle u^{\alpha}(p)\left[\bar{\kappa}(t+1, w)+\bar{p}^{\prime}\right]^{\alpha \beta} u_{\beta}(q)}{\langle p|v+1, \ldots, t| q\rangle\left\langle q|t+1, \ldots, w| p^{\prime}\right\rangle\left\langle q|w+1, \ldots, n| q^{\prime}\right\rangle} \\
+\frac{1}{N} i g^{n+2} & \sum_{\mathcal{P}(1 \ldots n)} \sum_{w=0}^{n} \sum_{t=0}^{w}(\Omega[1, w])^{i j}(\Omega[w+1, n])_{k \ell} \frac{1}{k_{0}^{2}} \mathcal{I}_{\alpha \dot{\alpha}}\left(0^{*}\right) \\
& \times \frac{(-\sqrt{2})^{n}\langle p q\rangle u^{\alpha}(p)\left[\bar{\kappa}(t+1, w)+\bar{p}^{\prime}\right]^{\dot{\alpha} \beta} u_{\beta}(q)}{\langle p|1, \ldots, t| q\rangle\left\langle q|t+1, \ldots, w| p^{\prime}\right\rangle\left\langle q|w+1, \ldots, n| q^{\prime}\right\rangle} .
\end{aligned}
$$

\subsection{Evaluation of the $1 / N$ contribution}

The simplest piece of (4.21) to evaluate is the $\frac{1}{N}$ contribution, which we will denote by $\mathcal{M}_{5}$. Inserting the explicit form (3.7) for $\mathcal{I}\left(0^{*}\right)$ produces

$$
\begin{aligned}
\mathcal{M}_{5}=\frac{i}{2 N}(-g \sqrt{2})^{n+2} \sum_{\mathcal{P}(1 \ldots n)} \sum_{w=0}^{n} \sum_{t=0}^{w}(\Omega[1, w])^{i j}(\Omega[w+1, n])_{k \ell} \\
\times \frac{1}{k_{0}^{2}} \frac{\langle p q\rangle^{2} u^{\gamma}(q) k_{0 \gamma \dot{\alpha}}\left[\bar{\kappa}(t+1, w)+\bar{p}^{\prime}\right]^{\dot{\alpha} \beta} u_{\beta}(q)}{\langle p|1, \ldots, t| q\rangle\left\langle q|t+1, \ldots, w| p^{\prime}\right\rangle\left\langle q|w+1, \ldots, n| q^{\prime}\right\rangle} .
\end{aligned}
$$

We note that the color factors are independent of $t$. In order to do the sum on $t$ in as compact a manner as possible we employ the following mnemonic device. We write the implicit $\kappa$ sum appearing in (4.22) as an explicit sum:

$$
\left[\bar{\kappa}(t+1, w)+\bar{p}^{\prime}\right]^{\dot{\alpha} \beta} \equiv \sum_{s=t+1}^{w+1} \bar{k}_{s}^{\dot{\alpha} \beta}
$$


with the understanding that when $s=w+1$ we write $p^{\prime}$ instead of $k_{w+1}$. The same kinds of considerations allow us to write

$$
\frac{1}{\langle p|1, \ldots, t| q\rangle\left\langle q|t+1, \ldots, w| p^{\prime}\right\rangle}=\frac{\langle t t+1\rangle}{\langle t|q| t+1\rangle} \frac{1}{\left\langle p|1, \ldots, w| p^{\prime}\right\rangle}
$$

for all values of $t$. In (4.24) we have the additional stipulation that when $t=0$ we should write $p$ rather than $k_{0}$. As a result, we do not have to spend the extra time to consider the endpoints of the summation separately, provided that we take care to follow the pattern set by (4.23) and (4.24). At first glance, this procedure may sound ad hoc; however, it is well-defined and always follows the pattern just outlined: when joining two denominator "strings", the correct value for "out of range" summation variables (i.e. those which are not included in the range of gluon momenta represented in the "string") is determined by the quark momentum appearing at the corresponding end of the "string". Since this type of structure occurs many times in this calculation, the savings generated by this trick is considerable.

Using (4.23) and (4.24), we see that (4.22) contains

$$
\begin{aligned}
\sigma_{5} & \equiv \sum_{t=0}^{w} \sum_{s=t+1}^{w+1} \frac{\langle t t+1\rangle}{\langle t|q| t+1\rangle} u^{\gamma}(q) k_{0 \gamma \dot{\alpha}} \bar{k}_{s}^{\dot{\alpha} \beta} u_{\beta}(q) \\
& =\sum_{s=1}^{w+1} \sum_{t=0}^{s-1} \frac{\langle t t+1\rangle}{\langle t|q| t+1\rangle} u^{\gamma}(q) k_{0 \gamma \dot{\alpha}} \bar{u}^{\dot{\alpha}}\left(k_{s}\right)\langle s q\rangle,
\end{aligned}
$$

where we have interchanged the order of the summations and used (A.11) to obtain the second line. We may perform the sum on $t$ using (A.16) to obtain

$$
\sigma_{5}=\sum_{s=1}^{w+1} \frac{\langle p s\rangle}{\langle p|q| s\rangle} u^{\gamma}(q) k_{0 \gamma \dot{\alpha}} \bar{u}^{\dot{\alpha}}\left(k_{s}\right)\langle s q\rangle,
$$

replacing $k_{t=0}$ with $p$ as described in the previous paragraph. Cancelling the common factor of $\langle s q\rangle$ from (4.26) allows us to do the remaining sum:

$$
\sigma_{5}=\frac{1}{\langle p q\rangle} u^{\gamma}(q) k_{0 \gamma \dot{\alpha}}\left[\bar{\kappa}(1, w)+\bar{p}^{\prime}\right]^{\dot{\alpha} \beta} u_{\beta}(p) .
$$


The Weyl equation permits us to add $\bar{p}^{\dot{\alpha} \beta}$ to the sum in (4.27) for free. But, according to (4.6), the resulting sum is just $-\bar{k}_{0}^{\dot{\alpha} \beta}$. Thus

$$
\begin{aligned}
\sigma_{5} & =-\frac{1}{\langle p q\rangle} u^{\gamma}(q) k_{0 \gamma \dot{\alpha}} k_{0}^{\dot{\alpha} \beta} u_{\beta}(p) \\
& =k_{0}^{2}
\end{aligned}
$$

where we have used (A.6). Putting (4.28) back into (4.22) gives

$$
\begin{aligned}
\mathcal{M}_{5}=\frac{i}{2 N}(-g \sqrt{2})^{n+2} \sum_{\mathcal{P}(1 \ldots n)} & \sum_{w=0}^{n}(\Omega[1, w])^{i j}(\Omega[w+1, n])_{k \ell} \\
& \times \frac{\langle p q\rangle^{2}}{\left\langle p|1, \ldots, w| p^{\prime}\right\rangle\left\langle q|w+1, \ldots, n| q^{\prime}\right\rangle} .
\end{aligned}
$$

\subsection{Evaluation of the $N$-independent contribution}

The $N$-independent contribution to the amplitude comes from the first term in (4.21). The seemingly complicated nature of the color factor appearing in this term suggests that the labelling scheme for the gluons appearing in Figure 1

is not optimal for this contribution. Instead, to obtain a simpler expression, we use the labels suggested by Figure 2. This makes the $N$-independent contribution read

$$
\begin{aligned}
& \mathcal{M}_{k \ell}^{i j}\left(p^{-}, q^{-} ; 1^{+}, \ldots, n^{+} ; p^{\prime+}, q^{\prime+}\right)= \\
&=-i g^{n+2} \sum_{\mathcal{P}(1 \ldots n)} \sum_{b=0}^{n} \sum_{c=b}^{n} \sum_{d=c}^{n} \sum_{e=d}^{n}(\Omega[1, d])^{i} \ell(\Omega[d+1, n])_{k}{ }^{j} \\
& \quad \times \frac{1}{k_{0}^{2}} \mathcal{I}_{\alpha \dot{\alpha}}\left((b+1)^{+}, \ldots, c^{+}, 0^{*},(d+1)^{+}, \ldots, e^{+}\right) \\
& \quad \times \frac{(-\sqrt{2})^{n+b-c+d-e}\langle p q\rangle u^{\alpha}(p)\left[\bar{\kappa}(e+1, n)+\bar{p}^{\prime}\right]^{\dot{\alpha} \beta} u_{\beta}(q)}{\langle p|1, \ldots, b| q\rangle\left\langle q|e+1, \ldots, n| p^{\prime}\right\rangle\left\langle q|c+1, \ldots, d| q^{\prime}\right\rangle},
\end{aligned}
$$

where the new limits are most easily obtained by examining Figure 2. The momentum of the zeroth gluon now reads

$$
\begin{aligned}
k_{0} & =q+q^{\prime}+\kappa(c+1, d) \\
& =-p-\kappa(1, c)-\kappa(d+1, n)-p^{\prime}
\end{aligned}
$$


Because the explicit form of the modified gluon current depends on the location of the gluon labelled by $0^{*}$, we write this term as the sum of four contributions. If $c=b$ and $e=d$, then $\mathcal{I}\left(0^{*}\right)$ appears:

$$
\begin{aligned}
\mathcal{M}_{1} \equiv-i g^{n+2} & \sum_{\mathcal{P}(1 \ldots n)} \sum_{b=0}^{n} \sum_{d=b}^{n}(\Omega[1, d])_{\ell}^{i}(\Omega[d+1, n])_{k}^{j} \\
& \times \frac{1}{k_{0}^{2}} \frac{(-\sqrt{2})^{n}\langle p q\rangle u^{\alpha}(p) \mathcal{I}_{\alpha \dot{\alpha}}\left(0^{*}\right)\left[\bar{\kappa}(d+1, n)+\bar{p}^{\prime}\right]^{\dot{\alpha} \beta} u_{\beta}(q)}{\langle p|1, \ldots, b| q\rangle\left\langle q|d+1, \ldots, n| p^{\prime}\right\rangle\left\langle q|b+1, \ldots, d| q^{\prime}\right\rangle} .
\end{aligned}
$$

This term corresponds to single gluon exchange. Next, we write the terms that have $0^{*}$ as the first of multiple arguments of $\mathcal{I}$. These are the remaining $c=b$ contributions:

$$
\begin{aligned}
\mathcal{M}_{2} \equiv-i g^{n+2} \sum_{\mathcal{P}(1 \ldots n)} & \sum_{b=0}^{n-1} \sum_{d=b}^{n-1} \sum_{e=d+1}^{n}(\Omega[1, d])_{\ell}^{i}(\Omega[d+1, n])_{k}^{j} \\
& \times \frac{1}{k_{0}^{2}} \mathcal{I}_{\alpha \dot{\alpha}}\left(0^{*},(d+1)^{+}, \ldots, e^{+}\right) \\
& \times \frac{(-\sqrt{2})^{n+d-e}\langle p q\rangle u^{\alpha}(p)\left[\bar{\kappa}(e+1, n)+\bar{p}^{\prime}\right]^{\dot{\alpha} \beta} u_{\beta}(q)}{\langle p|1, \ldots, b| q\rangle\left\langle q|e+1, \ldots, n| p^{\prime}\right\rangle\left\langle q|b+1, \ldots, d| q^{\prime}\right\rangle} .
\end{aligned}
$$

Note that we start at $e=d+1$ and adjust the $b$ and $d$ summation ranges accordingly, since $e=d$ (with $c=b$ ) is already given in (4.32). The third piece contains multiple-argument $\mathcal{I}$ 's that have $0^{*}$ appearing last. These are the $e=d$ contributions not accounted for in the previous two terms:

$$
\begin{aligned}
\mathcal{M}_{3} \equiv-i g^{n+2} \sum_{\mathcal{P}(1 \ldots n)} & \sum_{b=0}^{n-1} \sum_{c=b+1}^{n} \sum_{d=c}^{n}(\Omega[1, d])^{i}{ }_{\ell}(\Omega[d+1, n])_{k}{ }^{j} \\
& \times \frac{1}{k_{0}^{2}} \mathcal{I}_{\alpha \dot{\alpha}}\left((b+1)^{+}, \ldots, c^{+}, 0^{*}\right) \\
& \times \frac{(-\sqrt{2})^{n+b-c}\langle p q\rangle u^{\alpha}(p)\left[\bar{\kappa}(d+1, n)+\bar{p}^{\prime}\right]^{\dot{\alpha} \beta} u_{\beta}(q)}{\langle p|1, \ldots, b| q\rangle\left\langle q|d+1, \ldots, n| p^{\prime}\right\rangle\left\langle q|c+1, \ldots, d| q^{\prime}\right\rangle}
\end{aligned}
$$

The sum on $c$ begins at $b+1$ since $c=b$ (with $e=d$ ) appears in (4.32). As a consequence, the range of $b$ must be adjusted. In all of the remaining terms, $0^{*}$ 
is neither the first nor the last argument of $\mathcal{I}$ :

$$
\begin{aligned}
\mathcal{M}_{4} \equiv-i g^{n+2} \sum_{\mathcal{P}(1 \ldots n)} & \sum_{b=0}^{n-2} \sum_{c=b+1}^{n-1} \sum_{d=c}^{n-1} \sum_{e=d+1}^{n}(\Omega[1, d])^{i}{ }_{\ell}(\Omega[d+1, n])_{k}{ }^{j} \\
& \times \frac{1}{k_{0}^{2}} \mathcal{I}_{\alpha \dot{\alpha}}\left((b+1)^{+}, \ldots, c^{+}, 0^{*},(d+1)^{+}, \ldots, e^{+}\right) \\
& \times \frac{(-\sqrt{2})^{n+b-c+d-e}\langle p q\rangle u^{\alpha}(p)\left[\bar{\kappa}(e+1, n)+\bar{p}^{\prime}\right]^{\dot{\alpha} \beta} u_{\beta}(q)}{\langle p|1, \ldots, b| q\rangle\left\langle q|e+1, \ldots, n| p^{\prime}\right\rangle\left\langle q|c+1, \ldots, d| q^{\prime}\right\rangle} .
\end{aligned}
$$

Once more we have adjusted the summation ranges to avoid contributions already accounted for. We will evaluate each of $\mathcal{M}_{1}$ through $\mathcal{M}_{4}$ in order.

\subsubsection{Evaluation of $\mathcal{M}_{1}$}

We insert the expression (3.7) for $\mathcal{I}\left(0^{*}\right)$ into equation (4.32) to obtain the contribution from $\mathcal{M}_{1}$ :

$$
\begin{aligned}
\mathcal{M}_{1}=\frac{-i}{2}(-g \sqrt{2})^{n+2} \sum_{\mathcal{P}(1 \ldots n)} \sum_{b=0}^{n} \sum_{d=b}^{n}(\Omega[1, d])^{i}(\Omega[d+1, n])_{k}{ }^{j} \\
\times \frac{1}{k_{0}^{2}} \frac{\langle p q\rangle^{2} u^{\alpha}(q) k_{0 \alpha \dot{\alpha}}\left[\bar{\kappa}(d+1, n)+\bar{p}^{\prime}\right]^{\dot{\alpha} \beta} u_{\beta}(q)}{\langle p|1, \ldots, b| q\rangle\left\langle q|b+1, \ldots, d| q^{\prime}\right\rangle\left\langle q|d+1, \ldots, n| p^{\prime}\right\rangle} .
\end{aligned}
$$

Using (4.31) with $c=b$ we see that the numerator in (4.36) reads

$$
\begin{aligned}
\mathcal{N}_{1} & \equiv u^{\alpha}(q) k_{0 \alpha \dot{\alpha}}\left[\bar{\kappa}(d+1, n)+\bar{p}^{\prime}\right]^{\dot{\alpha} \beta} u_{\beta}(q) \\
& =-u^{\alpha}(q)\left[p+\kappa(1, b)+\kappa(d+1, n)+p^{\prime}\right]_{\alpha \dot{\alpha}}\left[\bar{\kappa}(d+1, n)+\bar{p}^{\prime}\right]^{\dot{\alpha} \beta} u_{\beta}(q) \\
& =-u^{\alpha}(q)[p+\kappa(1, b)]_{\alpha \dot{\alpha}}\left[\bar{\kappa}(d+1, n)+\bar{p}^{\prime}\right]^{\dot{\alpha} \beta} u_{\beta}(q),
\end{aligned}
$$

where we have used (A.6) to extract a term $\left[\kappa(d+1, n)+p^{\prime}\right]^{2}\langle q q\rangle=0$. Inserting (4.37) into (4.36) and interchanging the order of the summations produces

$$
\begin{aligned}
\mathcal{M}_{1}=\frac{i}{2}(-g \sqrt{2})^{n+2} & \sum_{\mathcal{P}(1 \ldots n)} \sum_{d=0}^{n} \sum_{b=0}^{d}(\Omega[1, d])^{i}(\Omega[d+1, n])_{k}{ }^{j} \\
& \times \frac{\langle p q\rangle^{2} u^{\alpha}(q)[p+\kappa(1, b)]_{\alpha \dot{\alpha}}\left[\bar{\kappa}(d+1, n)+\bar{p}^{\prime}\right]^{\dot{\alpha} \beta} u_{\beta}(q)}{\langle p|1, \ldots, b| q\rangle\left\langle q|b+1, \ldots, d| q^{\prime}\right\rangle\left\langle q|d+1, \ldots, n| p^{\prime}\right\rangle} \\
& \times \frac{1}{\left[q+q^{\prime}+\kappa(b+1, d)\right]^{2}} .
\end{aligned}
$$


No further simplification of $\mathcal{M}_{1}$ is possible because the propagator depends on both $b$ and $d$.

\subsubsection{Evaluation of $\mathcal{M}_{2}$}

Equation (4.33) for $\mathcal{M}_{2}$ contains $\mathcal{I}\left(0^{*},(d+1)^{+}, \ldots, e^{+}\right)$for $e \geq d+1$. Application of (3.46) and (3.47) produces

$$
\begin{aligned}
\mathcal{M}_{2}=\frac{i}{2} & (-g \sqrt{2})^{n+2} \sum_{\mathcal{P}(1 \ldots n)} \sum_{b=0}^{n-1} \sum_{d=b}^{n-1} \sum_{e=d+1}^{n} \sum_{y=d+1}^{e}(\Omega[1, d])^{i}{ }_{\ell}(\Omega[d+1, n])_{k}{ }^{j} \\
& \times \frac{\langle p q\rangle^{2} u^{\alpha}(q)\left[k_{0}+\kappa(d+1, e)\right]_{\alpha \dot{\alpha}}\left[\bar{\kappa}(e+1, n)+\bar{p}^{\prime}\right]^{\dot{\alpha} \beta} u_{\beta}(q)}{\langle p|1, \ldots, b| q\rangle\left\langle q|b+1, \ldots, d| q^{\prime}\right\rangle\langle q|d+1, \ldots, e| q\rangle\left\langle q|e+1, \ldots, n| p^{\prime}\right\rangle} \\
& \times c(0, d+1, \ldots, y) .
\end{aligned}
$$

We begin by observing that

$$
\begin{aligned}
c(0, d+1, \ldots, y) & =\frac{u^{\gamma}(q)\left[k_{0}+\kappa(d+1, y)\right]_{\gamma \dot{\gamma}} \bar{k}_{y}^{\dot{\gamma} \delta} u_{\delta}(q)}{\left[k_{0}+\kappa(d+1, y-1)\right]^{2}\left[k_{0}+\kappa(d+1, y)\right]^{2}} \\
& =\frac{u^{\gamma}(q)\left[q+q^{\prime}+\kappa(b+1, y)\right]_{\gamma \dot{\gamma}} \bar{k}_{y}^{\dot{\gamma} \delta} u_{\delta}(q)}{\left[q+q^{\prime}+\kappa(b+1, y-1)\right]^{2}\left[q+q^{\prime}+\kappa(b+1, y)\right]^{2}} \\
& =c\left(q+q^{\prime}, b+1, \ldots, y\right),
\end{aligned}
$$

as defined by (3.48) and in accordance with (4.31). The numerator of (4.39) may be written as

$$
\begin{aligned}
\mathcal{N}_{2} & \equiv u^{\alpha}(q)\left[k_{0}+\kappa(d+1, e)\right]_{\alpha \dot{\alpha}}\left[\bar{\kappa}(e+1, n)+\bar{p}^{\prime}\right]^{\dot{\alpha} \beta} u_{\beta}(q) \\
& =u^{\alpha}(q)\left[q+q^{\prime}+\kappa(b+1, e)\right]_{\alpha \dot{\alpha}}\left[\bar{\kappa}(e+1, n)+\bar{p}^{\prime}\right]^{\dot{\alpha} \beta} u_{\beta}(q) .
\end{aligned}
$$

We use (A.6) and momentum conservation to rewrite (4.41) as

$$
\begin{aligned}
\mathcal{N}_{2} & =u^{\alpha}(q)\left[q+q^{\prime}+\kappa(b+1, n)+p^{\prime}\right]_{\alpha \dot{\alpha}}\left[\bar{\kappa}(e+1, n)+\bar{p}^{\prime}\right]^{\dot{\alpha} \beta} u_{\beta}(q) \\
& =-u^{\alpha}(q)[p+\kappa(1, b)]_{\alpha \dot{\alpha}}\left[\bar{\kappa}(e+1, n)+\bar{p}^{\prime}\right]^{\dot{\alpha} \beta} u_{\beta}(q) .
\end{aligned}
$$

Inserting (4.40) and (4.42) back into (4.39) yields

$$
\begin{aligned}
& \mathcal{M}_{2}= \frac{-i}{2} \\
&(-g \sqrt{2})^{n+2} \sum_{\mathcal{P}(1 \ldots n)} \sum_{b=0}^{n-1} \sum_{d=b}^{n-1} \sum_{e=d+1}^{n} \sum_{y=d+1}^{e}(\Omega[1, d])^{i}{ }_{\ell}(\Omega[d+1, n])_{k}{ }^{j} \\
& \times \frac{\langle p q\rangle^{2} u^{\alpha}(q)[p+\kappa(1, b)]_{\alpha \dot{\alpha}}\left[\bar{\kappa}(e+1, n)+\bar{p}^{\prime}\right]^{\dot{\alpha} \beta} u_{\beta}(q)}{\langle p|1, \ldots, b| q\rangle\left\langle q|b+1, \ldots, d| q^{\prime}\right\rangle\langle q|d+1, \ldots, e| q\rangle\left\langle q|e+1, \ldots, n| p^{\prime}\right\rangle} \\
& \times c\left(q+q^{\prime}, b+1, \ldots, y\right) .
\end{aligned}
$$


At this stage we may perform the sum on $e$. Isolating the $e$-dependent portion of (4.43) we have

$$
\sigma_{2} \equiv \sum_{e=d+1}^{n} \sum_{y=d+1}^{e} \sum_{f=e+1}^{n+1} \frac{\langle e e+1\rangle}{\langle e|q| e+1\rangle} \bar{k}_{f}^{\dot{\alpha} \beta} u_{\beta}(q)
$$

where $k_{d}$ is understood to mean $q$ and $k_{n+1}$ really means $p^{\prime}$. The factor $\frac{\langle e e+1\rangle}{\langle e|q| e+1\rangle}$ allows us to write $\left\langle q|d+1, \ldots, n| p^{\prime}\right\rangle$ in the denominator of $\mathcal{M}_{2}$. Interchanging the order of the sums and using (A.16) produces

$$
\begin{aligned}
\sigma_{2} & =\sum_{y=d+1}^{n} \sum_{f=y+1}^{n+1} \sum_{e=y}^{f-1} \frac{\langle e e+1\rangle}{\langle e|q| e+1\rangle} \bar{u}^{\dot{\alpha}}\left(k_{f}\right)\langle f q\rangle \\
& =\sum_{y=d+1}^{n} \sum_{f=y+1}^{n+1} \frac{\langle y f\rangle}{\langle y|q| f\rangle} \bar{u}^{\dot{\alpha}}\left(k_{f}\right)\langle f q\rangle \\
& =\sum_{y=d+1}^{n} \frac{1}{\langle y q\rangle}\left[\bar{\kappa}(y+1, n)+\bar{p}^{\prime}\right]^{\dot{\alpha} \beta} u_{\alpha}\left(k_{y}\right) .
\end{aligned}
$$

When we insert (4.45) back into (4.43) and re-order the sums, we obtain

$$
\begin{aligned}
\mathcal{M}_{2}=\frac{-i}{2} & (-g \sqrt{2})^{n+2} \sum_{\mathcal{P}(1 \ldots n)} \sum_{d=0}^{n-1} \sum_{b=0}^{d} \sum_{y=d+1}^{n}(\Omega[1, d])^{i} \ell_{\ell}(\Omega[d+1, n])_{k}{ }^{j} \\
& \times \frac{\langle p q\rangle^{2} u^{\alpha}(q)[p+\kappa(1, b)]_{\alpha \dot{\alpha}}\left[\bar{\kappa}(y+1, n)+\bar{p}^{\prime}\right]^{\dot{\alpha} \beta} u_{\beta}\left(k_{y}\right)}{\langle p|1, \ldots, b| q\rangle\left\langle q|b+1, \ldots, d| q^{\prime}\right\rangle\left\langle q|d+1, \ldots, n| p^{\prime}\right\rangle} \\
& \times \frac{1}{\langle y q\rangle} c\left(q+q^{\prime}, b+1, \ldots, y\right) .
\end{aligned}
$$

Let us examine some of the factors in (4.46), namely the combination

$$
\Xi_{2} \equiv \frac{1}{\langle y q\rangle} u^{\alpha}(q)[p+\kappa(1, b)]_{\alpha \dot{\alpha}}\left[\bar{\kappa}(y+1, n)+\bar{p}^{\prime}\right]^{\dot{\alpha} \beta} u_{\beta}\left(k_{y}\right) c\left(q+q^{\prime}, b+1, \ldots, y\right)
$$

Supplying the definition of $c$ from (3.48) yields

$$
\Xi_{2}=\frac{u^{\alpha}(q)[p+\kappa(1, b)]_{\alpha \dot{\alpha}}\left[\bar{\kappa}(y+1, n)+\bar{p}^{\prime}\right]^{\dot{\alpha} \beta} k_{y \beta \dot{\beta}}\left[\bar{q}+\bar{q}^{\prime}+\bar{\kappa}(b+1, y)\right]^{\dot{\beta} \gamma} u_{\gamma}(q)}{\left[q+q^{\prime}+\kappa(b+1, y-1)\right]^{2}\left[q+q^{\prime}+\kappa(b+1, y)\right]^{2}}
$$


We may rewrite the numerator appearing in (4.48) as a combination of terms containing perfect squares. The key ingredients are using the Weyl equation to extend or shorten sums as appropriate, applying momentum conservation to factors, and adding zero (cleverly written) to various factors. For example, using the Weyl equation to write $\bar{\kappa}(y, n)$ for $\bar{\kappa}(y+1, n)$ and writing

$$
k_{y}=\left[q+q^{\prime}+\kappa(b+1, y)\right]-\left[q+q^{\prime}+\kappa(b+1, y-1)\right]
$$

produces

$$
\begin{aligned}
\mathcal{N}_{2} \equiv & u^{\alpha}(q)[p+\kappa(1, b)]_{\alpha \dot{\alpha}}\left[\bar{\kappa}(y, n)+\bar{p}^{\prime}\right]^{\dot{\alpha} \beta} k_{y \beta \dot{\beta}}\left[\bar{q}+\bar{q}^{\prime}+\bar{\kappa}(b+1, y)\right]^{\dot{\beta} \gamma} u_{\gamma}(q) \\
= & {\left[q+q^{\prime}+\kappa(b+1, y)\right]^{2} u^{\alpha}(q)[p+\kappa(1, b)]_{\alpha \dot{\alpha}}\left[\bar{\kappa}(y, n)+\bar{p}^{\prime}\right]^{\dot{\alpha} \gamma} u_{\gamma}(q) } \\
& -u^{\alpha}(q)[p+\kappa(1, b)]_{\alpha \dot{\alpha}}\left[\bar{\kappa}(y, n)+\bar{p}^{\prime}\right]^{\dot{\alpha} \beta} \\
& \times\left[q+q^{\prime}+\kappa(b+1, y-1)\right]_{\beta \dot{\beta}}\left[\bar{q}+\bar{q}^{\prime}+\bar{\kappa}(b+1, y)\right]^{\dot{\beta} \gamma} u_{\gamma}(q) .
\end{aligned}
$$

Momentum conservation lets us write

$$
-\left[\kappa(y, n)+p^{\prime}\right]=[p+\kappa(1, b)]+\left[q+q^{\prime}+\kappa(b+1, y-1)\right],
$$

yielding

$$
\begin{aligned}
\mathcal{N}_{2}= & {\left[q+q^{\prime}+\kappa(b+1, y)\right]^{2} u^{\alpha}(q)[p+\kappa(1, b)]_{\alpha \dot{\alpha}}\left[\bar{\kappa}(y, n)+\bar{p}^{\prime}\right]^{\dot{\alpha} \gamma} u_{\gamma}(q) } \\
& +[p+\kappa(1, b)]^{2} u^{\alpha}(q)\left[q+q^{\prime}+\kappa(b+1, y-1)\right]_{\alpha \dot{\beta}}\left[\bar{q}+\bar{q}^{\prime}+\bar{\kappa}(b+1, y)\right]^{\dot{\beta} \gamma} u_{\gamma}(q) \\
& +\left[q+q^{\prime}+\kappa(b+1, y-1)\right]^{2} u^{\alpha}(q)[p+\kappa(1, b)]_{\alpha \dot{\beta}}\left[\bar{q}+\bar{q}^{\prime}+\bar{\kappa}(b+1, y)\right]^{\dot{\beta} \gamma} u_{\gamma}(q) .
\end{aligned}
$$

Finally, we use momentum conservation on the factor $\left[\bar{q}+\bar{q}^{\prime}+\bar{\kappa}(b+1, y)\right]^{\dot{\beta} \gamma}$ appearing in the third term and shorten some of the momentum sums by extracting pieces proportional to $\langle q q\rangle$. The result of this manipulation is

$$
\begin{aligned}
\mathcal{N}_{2}= & {\left[q+q^{\prime}+\kappa(b+1, y)\right]^{2} u^{\alpha}(q)[p+\kappa(1, b)]_{\alpha \dot{\alpha}}\left[\bar{\kappa}(y, n)+\bar{p}^{\prime}\right]^{\dot{\alpha} \gamma} u_{\gamma}(q) } \\
& -\left[q+q^{\prime}+\kappa(b+1, y-1)\right]^{2} u^{\alpha}(q)[p+\kappa(1, b)]_{\alpha \dot{\beta}}\left[\bar{\kappa}(y+1, n)+\bar{p}^{\prime}\right]^{\dot{\beta} \gamma} u_{\gamma}(q) \\
& +[p+\kappa(1, b)]^{2} u^{\alpha}(q)\left[q+q^{\prime}+\kappa(b+1, y-1)\right]_{\alpha \dot{\beta}} \bar{k}_{y}^{\dot{\beta} \gamma} u_{\gamma}(q),
\end{aligned}
$$


where we have grouped the terms in a suggestive manner.

When (4.53) is inserted into (4.48), we observe that there are denominator cancellations in the first two terms, and that the third term contains a factor of $c\left(q+q^{\prime}, b+1, \ldots, y\right):$

$$
\begin{aligned}
\Xi_{2}= & {\left[\frac{u^{\alpha}(q)[p+\kappa(1, b)]_{\alpha \dot{\alpha}}\left[\bar{\kappa}(y, n)+\bar{p}^{\prime}\right]^{\dot{\alpha} \gamma} u_{\gamma}(q)}{\left[q+q^{\prime}+\kappa(b+1, y-1)\right]^{2}}\right.} \\
& \left.-\frac{u^{\alpha}(q)[p+\kappa(1, b)]_{\alpha \dot{\beta}}\left[\bar{\kappa}(y+1, n)+\bar{p}^{\prime}\right]^{\dot{\beta} \gamma} u_{\gamma}(q)}{\left[q+q^{\prime}+\kappa(b+1, y)\right]^{2}}\right] \\
& +[p+\kappa(1, b)]^{2} c\left(q+q^{\prime}, b+1, \ldots, y\right) .
\end{aligned}
$$

Combining (4.54) with (4.46), we notice that the terms grouped in brackets may be immediately summed over $y$, giving

$$
\begin{aligned}
\mathcal{M}_{2}=-\frac{i}{2}( & -g \sqrt{2})^{n+2} \sum_{\mathcal{P}(1 \ldots n)} \sum_{d=0}^{n-1} \sum_{b=0}^{d}(\Omega[1, d])^{i} \ell_{\ell}(\Omega[d+1, n])_{k}{ }^{j} \\
& \times \frac{\langle p q\rangle^{2}}{\langle p|1, \ldots, b| q\rangle\left\langle q|b+1, \ldots, d| q^{\prime}\right\rangle\left\langle q|d+1, \ldots, n| p^{\prime}\right\rangle} \\
& \times\left[\frac{u^{\alpha}(q)[p+\kappa(1, b)]_{\alpha \dot{\alpha}}\left[\bar{\kappa}(d+1, n)+\bar{p}^{\prime}\right]^{\dot{\alpha} \gamma} u_{\gamma}(q)}{\left[q+q^{\prime}+\kappa(b+1, d)\right]^{2}}\right. \\
-\frac{i}{2}( & -g \sqrt{2})^{n+2} \sum_{\mathcal{P}(1 \ldots n)} \sum_{d=0}^{n-1} \sum_{b=0}^{d} \sum_{y=d+1}^{n}(\Omega[1, d])^{i}{ }_{\ell}(\Omega[d+1, n])_{k}{ }^{j} \\
& \times \frac{\langle p q\rangle^{2}[p+\kappa(1, b)]^{2} c\left(q+q^{\prime}, b+1, \ldots, y\right)}{\langle p|1, \ldots, b| q\rangle\left\langle q|b+1, \ldots, d| q^{\prime}\right\rangle\left\langle q|d+1, \ldots, n| p^{\prime}\right\rangle} .
\end{aligned}
$$

The quantity in square brackets in the first contribution to (4.55) vanishes when $d=n$; hence, we may extend the sum to include that point. So, the result for 
$\mathcal{M}_{2}$ reads

$$
\begin{aligned}
\mathcal{M}_{2}=-\frac{i}{2}(- & g \sqrt{2})^{n+2} \sum_{\mathcal{P}(1 \ldots n)} \sum_{d=0}^{n} \sum_{b=0}^{d}(\Omega[1, d])^{i}{ }_{\ell}(\Omega[d+1, n])_{k}{ }^{j} \\
& \times \frac{\langle p q\rangle^{2} u^{\alpha}(q)[p+\kappa(1, b)]_{\alpha \dot{\alpha}}\left[\bar{\kappa}(d+1, n)+\bar{p}^{\prime}\right]^{\dot{\alpha} \gamma} u_{\gamma}(q)}{\langle p|1, \ldots, b| q\rangle\left\langle q|b+1, \ldots, d| q^{\prime}\right\rangle\left\langle q|d+1, \ldots, n| p^{\prime}\right\rangle} \\
& \times \frac{1}{\left[q+q^{\prime}+\kappa(b+1, d)\right]^{2}} \\
+\frac{i}{2}(- & g \sqrt{2})^{n+2} \sum_{\mathcal{P}(1 \ldots n) \sum_{d=0}^{n} \sum_{b=0}^{d}(\Omega[1, d])^{i}{ }_{\ell}(\Omega[d+1, n])_{k}{ }^{j}} \\
& \times \frac{\langle p q\rangle^{2} u^{\alpha}(q)[p+\kappa(1, b)]_{\alpha \dot{\beta}} \bar{p}^{\prime} \dot{\beta} \gamma u_{\gamma}(q)}{\langle p|1, \ldots, b| q\rangle\left\langle q|b+1, \ldots, d| q^{\prime}\right\rangle\left\langle q|d+1, \ldots, n| p^{\prime}\right\rangle} \\
& \times \frac{1}{\left[p+p^{\prime}+\kappa(1, b)\right]^{2}} \\
-\frac{i}{2}(- & g \sqrt{2})^{n+2} \sum_{\mathcal{P}(1 \ldots n)}^{n-1} \sum_{d=0}^{b} \sum_{b=0}^{n} \sum_{y=d+1}^{n}(\Omega[1, d])^{i}{ }_{\ell}(\Omega[d+1, n])_{k}{ }^{j} \\
& \times \frac{\langle p q\rangle^{2}[p+\kappa(1, b)]^{2} c\left(q+q^{\prime}, b+1, \ldots, y\right)}{\langle p|1, \ldots, b| q\rangle\left\langle q|b+1, \ldots, d| q^{\prime}\right\rangle\left\langle q|d+1, \ldots, n| p^{\prime}\right\rangle} .
\end{aligned}
$$

Note that the first term in (4.56) exactly cancels the contribution from $\mathcal{M}_{1}$ ( $c f$. equation (4.38)).

\subsubsection{Evaluation of $\mathcal{M}_{3}$}

Equation (4.34) for $\mathcal{M}_{3}$ contains $\mathcal{I}\left((b+1)^{+}, \ldots, c^{+}, 0^{*}\right)$ for $c \geq b+1$. Application of (3.46) and (3.73) produces

$$
\begin{aligned}
\mathcal{M}_{3}=-\frac{i}{2} & -g \sqrt{2})^{n+2} \sum_{\mathcal{P}(1 \ldots n)} \sum_{b=0}^{n-1} \sum_{c=b+1}^{n} \sum_{d=c}^{n} \sum_{y=b+1}^{c}(\Omega[1, d])_{\ell}^{i}(\Omega[d+1, n])_{k}{ }^{j} \\
& \times \frac{\langle p q\rangle^{2} u^{\alpha}(q)\left[k_{0}+\kappa(b+1, c)\right]_{\alpha \dot{\alpha}}\left[\bar{\kappa}(d+1, n)+\bar{p}^{\prime}\right]^{\dot{\alpha} \beta} u_{\beta}(q)}{\langle p|1, \ldots, b| q\rangle\langle q|b+1, \ldots, c| q\rangle\left\langle q|c+1, \ldots, d| q^{\prime}\right\rangle\left\langle q|d+1, \ldots, n| p^{\prime}\right\rangle} \\
& \times c(0, c, c-1, \ldots, y) .
\end{aligned}
$$


The numerator of (4.57) contains

$$
\begin{aligned}
\mathcal{N}_{3} & \equiv u^{\alpha}(q)\left[k_{0}+\kappa(b+1, c)\right]_{\alpha \dot{\alpha}}\left[\bar{\kappa}(d+1, n)+\bar{p}^{\prime}\right]^{\dot{\alpha} \beta} u_{\beta}(q) \\
& =u^{\alpha}(q)\left[-p-\kappa(1, c)-\kappa(d+1, n)-p^{\prime}+\kappa(b+1, c)\right]_{\alpha \dot{\alpha}}\left[\bar{\kappa}(d+1, n)+\bar{p}^{\prime}\right]^{\dot{\alpha} \beta} u_{\beta}(q) \\
& =-u^{\alpha}(q)[p+\kappa(1, b)]_{\alpha \dot{\alpha}}\left[\bar{\kappa}(d+1, n)+\bar{p}^{\prime}\right]^{\dot{\alpha} \beta} u_{\beta}(q),
\end{aligned}
$$

where we have used (4.31) for $k_{0}$ and shortened the sum in the first factor by using (A.6) to remove $\left[\kappa(d+1, n)+p^{\prime}\right]^{2}\langle q q\rangle=0$. We note in passing that

$$
c(0, c, c-1, \ldots, y)=c\left(q+q^{\prime}, d, d-1, \ldots, y\right) .
$$

Inserting (4.58) and (4.59) back into (4.57) and re-ordering some of the sums produces

$$
\begin{aligned}
\mathcal{M}_{3}=\frac{i}{2}(- & g \sqrt{2})^{n+2} \sum_{\mathcal{P}(1 \ldots n)} \sum_{d=1}^{n} \sum_{y=1}^{d} \sum_{b=0}^{y-1} \sum_{c=y}^{d}(\Omega[1, d])_{\ell}^{i}(\Omega[d+1, n])_{k}^{j} \\
& \times \frac{\langle p q\rangle^{2} u^{\alpha}(q)[p+\kappa(1, b)]_{\alpha \dot{\alpha}}\left[\bar{\kappa}(d+1, n)+\bar{p}^{\prime}\right]^{\dot{\alpha} \beta} u_{\beta}(q)}{\langle p|1, \ldots, b| q\rangle\langle q|b+1, \ldots, c| q\rangle\left\langle q|c+1, \ldots, d| q^{\prime}\right\rangle\left\langle q|d+1, \ldots, n| p^{\prime}\right\rangle} \\
& \times c\left(q+q^{\prime}, d, d-1, \ldots, y\right) .
\end{aligned}
$$

The sum on $c$ appearing in (4.60) is almost trivial. Applying (A.16) produces

$$
\begin{aligned}
\mathcal{M}_{3}=\frac{i}{2}(- & g \sqrt{2})^{n+2} \sum_{\mathcal{P}(1 \ldots n)} \sum_{d=1}^{n} \sum_{y=1}^{d} \sum_{b=0}^{y-1}(\Omega[1, d])_{\ell}^{i}(\Omega[d+1, n])_{k}{ }^{j} \\
& \times \frac{\langle p q\rangle^{2} u^{\alpha}(q)[p+\kappa(1, b)]_{\alpha \dot{\alpha}}\left[\bar{\kappa}(d+1, n)+\bar{p}^{\prime}\right]^{\dot{\alpha} \beta} u_{\beta}(q)}{\langle p|1, \ldots, b| q\rangle\left\langle q|b+1, \ldots, d| q^{\prime}\right\rangle\left\langle q|d+1, \ldots, n| p^{\prime}\right\rangle} \\
& \times \frac{\left\langle y q^{\prime}\right\rangle}{\left\langle y|q| q^{\prime}\right\rangle} c\left(q+q^{\prime}, d, d-1, \ldots, y\right) .
\end{aligned}
$$

The key factors in (4.61),

$$
\sigma_{3} \equiv \sum_{b=0}^{y-1} \sum_{a=0}^{b} u^{\alpha}(q) k_{a \alpha \dot{\alpha}} \frac{\langle b b+1\rangle}{\langle b|q| b+1\rangle},
$$

are summed following the procedure used on $\sigma_{2}$ (cf. equations (4.44)-(4.45)). The result is

$$
\sigma_{3}=\frac{1}{\langle q y\rangle} u^{\alpha}\left(k_{y}\right)[p+\kappa(1, y-1)]_{\alpha \dot{\alpha}}
$$


Inserting (4.63) into (4.61) results in

$$
\begin{aligned}
\mathcal{M}_{3}=\frac{i}{2}(- & g \sqrt{2})^{n+2} \sum_{\mathcal{P}(1 \ldots n)} \sum_{d=1}^{n} \sum_{y=1}^{d}(\Omega[1, d])_{\ell}^{i}(\Omega[d+1, n])_{k}{ }^{j} \\
& \times \frac{\langle p q\rangle^{2} u^{\alpha}\left(k_{y}\right)[p+\kappa(1, y-1)]_{\alpha \dot{\alpha}}\left[\bar{\kappa}(d+1, n)+\bar{p}^{\prime}\right]^{\dot{\alpha} \beta} u_{\beta}(q)}{\left\langle p|1, \ldots, d| q^{\prime}\right\rangle\left\langle q|d+1, \ldots, n| p^{\prime}\right\rangle} \\
& \times \frac{\left\langle y q^{\prime}\right\rangle}{\langle y q\rangle\left\langle q q^{\prime}\right\rangle\langle q y\rangle} c\left(q+q^{\prime}, d, d-1, \ldots, y\right) .
\end{aligned}
$$

Momentum conservation allows us to split (4.64) into two contributions by writing

$$
[p+\kappa(1, y-1)]_{\alpha \dot{\alpha}}=-\left[\kappa(d+1, n)+p^{\prime}\right]_{\alpha \dot{\alpha}}-\left[q+q^{\prime}+\kappa(y, d)\right]_{\alpha \dot{\alpha}}
$$

The first term of (4.65) produces

$$
\begin{aligned}
\mathcal{M}_{3 \mathrm{~A}} \equiv-\frac{i}{2} & (-g \sqrt{2})^{n+2} \sum_{\mathcal{P}(1 \ldots n)} \sum_{d=1}^{n} \sum_{y=1}^{d}(\Omega[1, d])^{i}{ }_{\ell}(\Omega[d+1, n])_{k}{ }^{j} \\
& \times \frac{\langle p q\rangle^{2}\left[\kappa(d+1, n)+p^{\prime}\right]^{2}}{\left\langle p|1, \ldots, d| q^{\prime}\right\rangle\left\langle q|d+1, \ldots, n| p^{\prime}\right\rangle} \\
& \times \frac{\left\langle y q^{\prime}\right\rangle}{\left\langle q q^{\prime}\right\rangle\langle q y\rangle} c\left(q+q^{\prime}, d, d-1, \ldots, y\right),
\end{aligned}
$$

which we set aside for the moment. Later, we will find that this contribution is cancelled by a portion of $\mathcal{M}_{4}$. The other term generated by (4.65) produces

$$
\begin{aligned}
\mathcal{M}_{3 \mathrm{~B}} \equiv-\frac{i}{2} & (-g \sqrt{2})^{n+2} \sum_{\mathcal{P}(1 \ldots n)} \sum_{d=1}^{n} \sum_{y=1}^{d}(\Omega[1, d])_{\ell}^{i}(\Omega[d+1, n])_{k}{ }^{j} \\
& \times \frac{\langle p q\rangle^{2} u^{\alpha}\left(k_{y}\right)\left[q+q^{\prime}+\kappa(y, d)\right]_{\alpha \dot{\alpha}}\left[\bar{\kappa}(d+1, n)+\bar{p}^{\prime}\right]^{\dot{\alpha} \beta} u_{\beta}(q)}{\left\langle p|1, \ldots, d| q^{\prime}\right\rangle\left\langle q|d+1, \ldots, n| p^{\prime}\right\rangle} \\
& \times \frac{\left\langle y q^{\prime}\right\rangle}{\langle y q\rangle\left\langle q q^{\prime}\right\rangle\langle q y\rangle} c\left(q+q^{\prime}, d, d-1, \ldots, y\right) .
\end{aligned}
$$

At this stage we would like to treat

$$
\begin{aligned}
\Xi_{3 \mathrm{~B}} \equiv & u^{\alpha}\left(k_{y}\right)\left[q+q^{\prime}+\kappa(y, d)\right]_{\alpha \dot{\alpha}}\left[\bar{\kappa}(d+1, n)+\bar{p}^{\prime}\right]^{\dot{\alpha} \beta} u_{\beta}(q) \\
& \times \frac{\left\langle y q^{\prime}\right\rangle}{\langle y q\rangle\left\langle q q^{\prime}\right\rangle\langle q y\rangle} c\left(q+q^{\prime}, d, d-1, \ldots, y\right)
\end{aligned}
$$


in the same manner as $\Xi_{2}$. Thus, we insert the definition (3.48) of $c$ into (4.68) to obtain

$$
\begin{aligned}
\Xi_{3 \mathrm{~B}}= & \frac{u^{\delta}(q)\left[q+q^{\prime}+\kappa(y, d)\right]_{\delta \dot{\delta}} \bar{k}_{y}^{\dot{\delta} \alpha}\left[q+q^{\prime}+\kappa(y+1, d)\right]_{\alpha \dot{\alpha}}\left[\bar{\kappa}(d+1, n)+\bar{p}^{\prime}\right]^{\dot{\alpha} \beta} u_{\beta}(q)}{\left[q+q^{\prime}+\kappa(y+1, d)\right]^{2}\left[q+q^{\prime}+\kappa(y, d)\right]^{2}} \\
& \times \frac{\left\langle y q^{\prime}\right\rangle}{\left\langle q q^{\prime}\right\rangle\langle q y\rangle}
\end{aligned}
$$

We use

$$
k_{y}=\left[q+q^{\prime}+\kappa(y, d)\right]-\left[q+q^{\prime}+\kappa(y+1, d)\right]
$$

to rewrite the numerator of (4.69):

$$
\begin{aligned}
\mathcal{N}_{3 \mathrm{~B}} \equiv & u^{\delta}(q)\left[q+q^{\prime}+\kappa(y, d)\right]_{\delta \dot{\delta}} \bar{k}_{y}^{\dot{\delta} \alpha}\left[q+q^{\prime}+\kappa(y+1, d)\right]_{\alpha \dot{\alpha}}\left[\bar{\kappa}(d+1, n)+\bar{p}^{\prime}\right]^{\dot{\alpha} \beta} u_{\beta}(q) \\
= & {\left[q+q^{\prime}+\kappa(y, d)\right]^{2} u^{\delta}(q)\left[q+q^{\prime}+\kappa(y+1, d)\right]_{\delta \dot{\alpha}}\left[\bar{\kappa}(d+1, n)+\bar{p}^{\prime}\right]^{\dot{\alpha} \beta} u_{\beta}(q) } \\
& -\left[q+q^{\prime}+\kappa(y+1, d)\right]^{2} u^{\delta}(q)\left[q+q^{\prime}+\kappa(y, d)\right]_{\delta \dot{\delta}}\left[\bar{\kappa}(d+1, n)+\bar{p}^{\prime}\right]^{\dot{\delta} \beta} u_{\beta}(q) .
\end{aligned}
$$

Thus,

$$
\begin{aligned}
\Xi_{3 \mathrm{~B}}=\frac{\left\langle y q^{\prime}\right\rangle}{\left\langle q q^{\prime}\right\rangle\langle q y\rangle} & {\left[\frac{u^{\delta}(q)\left[q+q^{\prime}+\kappa(y+1, d)\right]_{\delta \dot{\delta}}}{\left[q+q^{\prime}+\kappa(y+1, d)\right]^{2}}\right.} \\
& \left.-\frac{u^{\delta}(q)\left[q+q^{\prime}+\kappa(y, d)\right]_{\delta \dot{\delta}}}{\left[q+q^{\prime}+\kappa(y, d)\right]^{2}}\right]\left[\bar{\kappa}(d+1, n)+\bar{p}^{\prime}\right]^{\dot{\delta} \beta} u_{\beta}(q) .
\end{aligned}
$$

It is useful to consider the sum on $y$ pending in the expression for $\mathcal{M}_{3 \mathrm{~B}}$. All of the $y$-dependence is contained in $\Xi_{3 \mathrm{~B}}$; the sum may be written as

$$
\sum_{y=1}^{d} \Xi_{3 \mathrm{~B}}=\sum_{y=1}^{d} \frac{\left\langle y q^{\prime}\right\rangle}{\left\langle q q^{\prime}\right\rangle\langle q y\rangle}[f(y+1)-f(y)]
$$

with $f(y)$ given by

$$
f(y) \equiv \frac{u^{\delta}(q)\left[q+q^{\prime}+\kappa(y, d)\right]_{\delta \dot{\delta}}\left[\bar{\kappa}(d+1, n)+\bar{p}^{\prime}\right]^{\dot{\delta} \beta} u_{\beta}(q)}{\left[q+q^{\prime}+\kappa(y, d)\right]^{2}} .
$$

Shifting the second sum by one produces

$$
\sum_{y=1}^{d} \Xi_{3 \mathrm{~B}}=-\sum_{y=1}^{d} \frac{\left\langle y q^{\prime}\right\rangle}{\left\langle y|q| q^{\prime}\right\rangle} f(y+1)-\sum_{y=0}^{d-1} \frac{\left\langle q^{\prime} y+1\right\rangle}{\left\langle q^{\prime}|q| y+1\right\rangle} f(y+1) .
$$


The two terms in (4.75) may be combined using (A.15), with the exception of the endpoints of the summation:

$$
\sum_{y=1}^{d} \Xi_{3 \mathrm{~B}}=-\sum_{y=1}^{d-1} \frac{\langle y y+1\rangle}{\langle y|q| y+1\rangle} f(y+1)-\frac{\left\langle d q^{\prime}\right\rangle}{\left\langle d|q| q^{\prime}\right\rangle} f(d+1)-\frac{\left\langle q^{\prime} 1\right\rangle}{\left\langle q^{\prime}|q| 1\right\rangle} f(1) .
$$

At this stage, we recall that the "natural" assignment for $k_{d+1}$ is $q^{\prime}$, according to the rules outlined in section 4.3. Thus, the second term in (4.76) may be absorbed into the first, giving

$$
\sum_{y=1}^{d} \Xi_{3 \mathrm{~B}}=-\sum_{y=1}^{d} \frac{\langle y y+1\rangle}{\langle y|q| y+1\rangle} f(y+1)+\frac{\left\langle 1 q^{\prime}\right\rangle}{\left\langle 1|q| q^{\prime}\right\rangle} f(1) .
$$

Before combining (4.67), (4.74), and (4.77), we note that the numerator of $f(y+1)$ may be rewritten as

$$
\begin{array}{r}
u^{\delta}(q)\left[q+q^{\prime}+\kappa(y+1, d)\right]_{\delta \dot{\delta}}\left[\bar{\kappa}(d+1, n)+\bar{p}^{\prime}\right]^{\dot{\delta} \beta} u_{\beta}(q)= \\
=-u^{\delta}(q)[p+\kappa(1, y)]_{\delta \dot{\delta}}\left[\bar{\kappa}(d+1, n)+\bar{p}^{\prime}\right]^{\dot{\delta} \beta} u_{\beta}(q)
\end{array}
$$

by applying momentum conservation and (A.6). Hence, our final result for $\mathcal{M}_{3 \mathrm{~B}}$ reads

$$
\begin{aligned}
\mathcal{M}_{3 \mathrm{~B}}=-\frac{i}{2} & -g \sqrt{2})^{n+2} \sum_{\mathcal{P}(1 \ldots n)} \sum_{d=1}^{n} \sum_{y=1}^{d}(\Omega[1, d])^{i} \ell(\Omega[d+1, n])_{k}^{j} \\
& \times \frac{\langle p q\rangle^{2} u^{\alpha}(q)[p+\kappa(1, y)]_{\alpha \dot{\alpha}}\left[\bar{\kappa}(d+1, n)+\bar{p}^{\prime}\right]^{\dot{\alpha} \beta} u_{\beta}(q)}{\langle p|1, \ldots, y| q\rangle\left\langle q|y+1, \ldots, d| q^{\prime}\right\rangle\left\langle q|d+1, \ldots, n| p^{\prime}\right\rangle} \\
& \times \frac{1}{\left[q+q^{\prime}+\kappa(y+1, d)\right]^{2}} \\
+ & \frac{i}{2}(-g \sqrt{2})^{n+2} \sum_{\mathcal{P}(1 \ldots n)}^{n} \sum_{d=1}^{n}(\Omega[1, d])^{i} \ell(\Omega[d+1, n])_{k}^{j} \\
& \times \frac{\langle p q\rangle^{2} u^{\alpha}(q) p_{\alpha \dot{\alpha}}\left[\bar{\kappa}(d+1, n)+\bar{p}^{\prime}\right]^{\dot{\alpha} \beta} u_{\beta}(q)}{\left\langle p|1, \ldots, d| q^{\prime}\right\rangle\left\langle q|d+1, \ldots, n| p^{\prime}\right\rangle} \\
& \times \frac{\left\langle 1 q^{\prime}\right\rangle}{\left\langle 1|q| q^{\prime}\right\rangle} \frac{1}{\left[q+q^{\prime}+\kappa(1, d)\right]^{2}} .
\end{aligned}
$$




\subsubsection{Evaluation of $\mathcal{M}_{4}$}

We come at last to the final contribution, $\mathcal{M}_{4}$. As indicated by its definition $(4.35), \mathcal{M}_{4}$ contains $\mathcal{I}\left((b+1)^{+}, \ldots, c^{+}, 0^{*},(d+1)^{+}, \ldots, e^{+}\right)$. As a consequence, it is the most complicated piece which must be dealt with. However, as we shall see, the procedure is straightforward and employs the same techniques already discussed.

We start by inserting (3.46) and (3.70) into (4.35). After some regrouping, we obtain

$$
\begin{aligned}
\mathcal{M}_{4}=\frac{-i}{2} & (-g \sqrt{2})^{n+2} \sum_{\mathcal{P}(1 \ldots n)} \sum_{b=0}^{n-2} \sum_{c=b+1}^{n-1} \sum_{d=c}^{n-1} \sum_{e=d+1}^{n} \sum_{y=b+1}^{c} \sum_{z=d+1}^{e}(\Omega[1, d])^{i}{ }_{\ell}(\Omega[d+1, n])_{k}{ }^{j} \\
& \times \frac{\langle p q\rangle^{2} u^{\alpha}(q)\left[\kappa(b+1, c)+k_{0}+\kappa(d+1, e)\right]_{\alpha \dot{\alpha}}\left[\bar{\kappa}(e+1, n)+\bar{p}^{\prime}\right]^{\dot{\alpha} \beta} u_{\beta}(q)}{\langle p|1, \ldots, b| q\rangle\langle q|b+1, \ldots, c| q\rangle\left\langle q|c+1, \ldots, d| q^{\prime}\right\rangle} \\
& \times \frac{c_{2}(y, \ldots, c, 0, d+1, \ldots, z)}{\langle q|d+1, \ldots, e| q\rangle\left\langle q|e+1, \ldots, n| p^{\prime}\right\rangle} \frac{\langle y|q| z\rangle}{\langle y z\rangle}
\end{aligned}
$$

Equation (4.31) tells us that

$$
\kappa(b+1, c)+k_{0}+\kappa(d+1, e)=q+q^{\prime}+\kappa(b+1, e) .
$$

There are also the consequences of the " 0 " appearing in $c_{2}(y, \ldots, c, 0, d+1, \ldots, z)$ to consider. First, note that the ranges $\{y, \ldots, c\}$ and $\{d+1, \ldots, e\}$ always contain at least one element each. Hence, "0" is never the first or last argument of $c_{2}$. Now recall that $c_{2}$ is symmetric in all but its first and last arguments. As a consequence, we may replace the single argument " 0 " by the multiple arguments " $q+q^{\prime}, c+1, c+2, \ldots, d$ " implied by (4.31). Thus,

$$
\begin{aligned}
c_{2}(y, \ldots, c, 0, d+1, \ldots, z) & =c_{2}\left(y, \ldots, c, q+q^{\prime}, c+1, \ldots, d, d+1, \ldots, z\right) \\
& =c_{2}\left(y, q+q^{\prime}, y+1, \ldots, z\right),
\end{aligned}
$$


where we have taken advantage of the symmetry in the arguments to obtain the last line. Combination of (4.81) and (4.82) with (4.80) produces the following expression after interchanging some of the summations:

$$
\begin{aligned}
\mathcal{M}_{4}=\frac{-i}{2} & (-g \sqrt{2})^{n+2} \sum_{\mathcal{P}(1 \ldots n)} \sum_{b=0}^{n-2} \sum_{c=b+1}^{n-1} \sum_{d=c}^{n-1} \sum_{y=b+1}^{c} \sum_{z=d+1}^{n} \sum_{e=z}^{n}(\Omega[1, d])^{i} \ell_{\ell}(\Omega[d+1, n])_{k}{ }^{j} \\
& \times \frac{\langle p q\rangle^{2} u^{\alpha}(q)\left[q+q^{\prime}+\kappa(b+1, e)\right]_{\alpha \dot{\alpha}}\left[\bar{\kappa}(e+1, n)+\bar{p}^{\prime}\right]^{\dot{\alpha} \beta} u_{\beta}(q)}{\langle p|1, \ldots, b| q\rangle\langle q|b+1, \ldots, c| q\rangle\left\langle q|c+1, \ldots, d| q^{\prime}\right\rangle} \\
& \times \frac{c_{2}\left(y, q+q^{\prime}, y+1, \ldots, z\right)}{\langle q|d+1, \ldots, e| q\rangle\left\langle q|e+1, \ldots, n| p^{\prime}\right\rangle} \frac{\langle y|q| z\rangle}{\langle y z\rangle} .
\end{aligned}
$$

Consider the sum on $e$ that appears in (4.83). We examine the following factors that appear in $\mathcal{M}_{4}$ :

$$
\sigma_{4 e} \equiv \sum_{e=z}^{n} u^{\alpha}(q)\left[q+q^{\prime}+\kappa(b+1, e)\right]_{\alpha \dot{\alpha}}\left[\bar{\kappa}(e+1, n)+\bar{p}^{\prime}\right]^{\dot{\alpha} \beta} u_{\beta}(q) \frac{\langle e e+1\rangle}{\langle e|q| e+1\rangle}
$$

which allows us to join $\langle q|d+1, \ldots, e| q\rangle$ to $\left\langle q|e+1, \ldots, n| p^{\prime}\right\rangle$ with the understanding that $k_{n+1}$ is read as $p^{\prime}$. Because of (A.6) and the antisymmetry of the spinor inner product, we may extend the first sum in (4.84) to

$$
\left[q+q^{\prime}+\kappa(b+1, n)+p^{\prime}\right]_{\alpha \dot{\alpha}}=-[p+\kappa(1, b)]_{\alpha \dot{\alpha}}
$$

Applying this to (4.84) and writing the remaining $e$-dependent $\kappa$-sum explicitly produces

$$
\sigma_{4 e}=-\sum_{e=z}^{n} \sum_{f=e+1}^{n+1} u^{\alpha}(q)[p+\kappa(1, b)]_{\alpha \dot{\alpha}} \bar{k}_{f}^{\dot{\alpha} \beta} u_{\beta}(q) \frac{\langle e e+1\rangle}{\langle e|q| e+1\rangle} .
$$

Interchanging the order of summation and applying (A.16) as usual produces

$$
\begin{aligned}
\sigma_{4 e} & =-\sum_{f=z+1}^{n+1} \sum_{e=z}^{f-1} u^{\alpha}(q)[p+\kappa(1, b)]_{\alpha \dot{\alpha}} \bar{k}_{f}^{\dot{\alpha} \beta} u_{\beta}(q) \frac{\langle e e+1\rangle}{\langle e|q| e+1\rangle} \\
& =-\sum_{f=z+1}^{n+1} u^{\alpha}(q)[p+\kappa(1, b)]_{\alpha \dot{\alpha}} \bar{u}^{\dot{\alpha}}\left(k_{f}\right)\langle f q\rangle \frac{\langle z f\rangle}{\langle z|q| f\rangle} \\
& =\frac{-1}{\langle z q\rangle} u^{\alpha}(q)[p+\kappa(1, b)]_{\alpha \dot{\alpha}}\left[\bar{\kappa}(z, n)+\bar{p}^{\prime}\right]^{\dot{\alpha} \beta} u_{\beta}\left(k_{z}\right) .
\end{aligned}
$$


Inserting (4.87) back into (4.83) and rearranging the summations produces

$$
\begin{aligned}
\mathcal{M}_{4}=\frac{-i}{2} & (-g \sqrt{2})^{n+2} \sum_{\mathcal{P}(1 \ldots n)} \sum_{d=1}^{n-1} \sum_{b=0}^{d-1} \sum_{c=b+1}^{d} \sum_{y=b+1}^{c} \sum_{z=d+1}^{n}(\Omega[1, d])^{i}{ }_{\ell}(\Omega[d+1, n])_{k}{ }^{j} \\
& \times \frac{\langle p q\rangle^{2} u^{\alpha}(q)[p+\kappa(1, b)]_{\alpha \dot{\alpha}}\left[\bar{\kappa}(z+1, n)+\bar{p}^{\prime}\right]^{\dot{\alpha} \beta} u_{\beta}\left(k_{z}\right)}{\langle p|1, \ldots, b| q\rangle\langle q|b+1, \ldots, c| q\rangle\left\langle q|c+1, \ldots, d| q^{\prime}\right\rangle\left\langle q|d+1, \ldots, n| p^{\prime}\right\rangle} \\
& \times \frac{\langle y q\rangle}{\langle y z\rangle} c_{2}\left(y, q+q^{\prime}, y+1, \ldots, z\right) .
\end{aligned}
$$

Equation (4.88) has only "trivial" $c$-dependence; we supply a factor $\frac{\langle c c+1\rangle}{\langle c|q| c+1\rangle}$ to join the corresponding pair of denominator "strings" and write

$$
\begin{aligned}
\sum_{c=b+1}^{d} \sum_{y=b+1}^{c} \sum_{z=d+1}^{n} \frac{\langle c c+1\rangle}{\langle c|q| c+1\rangle} & =\sum_{y=b+1}^{d} \sum_{z=d+1}^{n} \sum_{c=y}^{d} \frac{\langle c c+1\rangle}{\langle c|q| c+1\rangle} \\
& =\sum_{y=b+1}^{d} \sum_{z=d+1}^{n} \frac{\left\langle y q^{\prime}\right\rangle}{\left\langle y|q| q^{\prime}\right\rangle}
\end{aligned}
$$

Hence, $\mathcal{M}_{4}$ becomes

$$
\begin{aligned}
\mathcal{M}_{4}=\frac{-i}{2} & (-g \sqrt{2})^{n+2} \sum_{\mathcal{P}(1 \ldots n)} \sum_{d=1}^{n-1} \sum_{b=0}^{d-1} \sum_{y=b+1}^{d} \sum_{z=d+1}^{n}(\Omega[1, d])^{i}{ }_{\ell}(\Omega[d+1, n])_{k}{ }^{j} \\
& \times \frac{\langle p q\rangle^{2} u^{\alpha}(q)[p+\kappa(1, b)]_{\alpha \dot{\alpha}}\left[\bar{\kappa}(z+1, n)+\bar{p}^{\prime}\right]^{\dot{\alpha} \beta} u_{\beta}\left(k_{z}\right)}{\langle p|1, \ldots, b| q\rangle\left\langle q|b+1, \ldots, d| q^{\prime}\right\rangle\left\langle q|d+1, \ldots, n| p^{\prime}\right\rangle} \\
& \times \frac{\left\langle y q^{\prime}\right\rangle}{\langle y z\rangle\left\langle q q^{\prime}\right\rangle} c_{2}\left(y, q+q^{\prime}, y+1, \ldots, z\right) .
\end{aligned}
$$

The sum on $b$ in (4.90) may be performed as well. The relevant factors are

$$
\sigma_{4 b} \equiv \sum_{b=0}^{d-1} \sum_{y=b+1}^{d} \sum_{z=d+1}^{n} \sum_{a=0}^{b} \frac{\langle b b+1\rangle}{\langle b|q| b+1\rangle} u^{\alpha}(q) k_{a \alpha \dot{\alpha}}
$$

where we have written the $b$-dependent $\kappa$-sum as a sum on $a, k_{0} \equiv p$. Interchanging the order of summation and applying (A.16), we obtain

$$
\begin{aligned}
\sigma_{4 b} & =\sum_{y=1}^{d} \sum_{z=d+1}^{n} \sum_{a=0}^{y-1} \sum_{b=a}^{y-1} \frac{\langle b b+1\rangle}{\langle b|q| b+1\rangle}\langle q a\rangle \bar{u}_{\dot{\alpha}}\left(k_{a}\right) \\
& =\sum_{y=1}^{d} \sum_{z=d+1}^{n} \frac{1}{\langle q y\rangle} u^{\alpha}\left(k_{y}\right)[p+\kappa(1, y-1)]_{\alpha \dot{\alpha}} .
\end{aligned}
$$


At this stage, we have

$$
\begin{aligned}
\mathcal{M}_{4}=\frac{-i}{2}(-g \sqrt{2})^{n+2} \sum_{\mathcal{P}(1 \ldots n)} \sum_{d=1}^{n-1} \sum_{y=1}^{d} \sum_{z=d+1}^{n}(\Omega[1, d])^{i} \ell(\Omega[d+1, n])_{k}^{j} \\
\times \frac{\langle p q\rangle^{2} u^{\alpha}\left(k_{y}\right)[p+\kappa(1, y-1)]_{\alpha \dot{\alpha}}\left[\bar{\kappa}(z+1, n)+\bar{p}^{\prime}\right]^{\dot{\alpha} \beta} u_{\beta}\left(k_{z}\right)}{\left\langle p|1, \ldots, d| q^{\prime}\right\rangle\left\langle q|d+1, \ldots, n| p^{\prime}\right\rangle} \\
\times \frac{\left\langle y q^{\prime}\right\rangle}{\langle q y\rangle\langle y z\rangle\left\langle q q^{\prime}\right\rangle}\left[c\left(q+q^{\prime}, y, y+1, \ldots, z\right)-c\left(q+q^{\prime}, y+1, y+2, \ldots, z\right)\right. \\
\left.\quad-c\left(q+q^{\prime}, z, z-1, \ldots, y\right)+c\left(q+q^{\prime}, z-1, z-2, \ldots, y\right)\right],
\end{aligned}
$$

where we have made use of (3.57) and the symmetry properties of the c's to write out $c_{2}\left(y, q+q^{\prime}, y+1, \ldots, z\right)$.

In order to make further progress with equation (4.93), we must insert explicit expressions for the $c$ functions. Let us begin by defining

$$
\begin{aligned}
\Xi_{4} \equiv \sum_{d=1}^{n-1} \sum_{y=1}^{d} \sum_{z=d+1}^{n} u^{\alpha}\left(k_{y}\right)[p+\kappa(1, y-1)]_{\alpha \dot{\alpha}}\left[\bar{\kappa}(z+1, n)+\bar{p}^{\prime}\right]^{\dot{\alpha} \beta} u_{\beta}\left(k_{z}\right) \\
\times \frac{\left\langle y q^{\prime}\right\rangle}{\langle q y\rangle\langle y z\rangle\left\langle q q^{\prime}\right\rangle}\left[c\left(q+q^{\prime}, y, y+1, \ldots, z\right)-c\left(q+q^{\prime}, y+1, y+2, \ldots, z\right)\right. \\
\left.-c\left(q+q^{\prime}, z, z-1, \ldots, y\right)+c\left(q+q^{\prime}, z-1, z-2, \ldots, y\right)\right] .
\end{aligned}
$$

We have retained the sum on $d$ since we will be adjusting its range at a later stage. It is understood that there are $d$-dependent factors appearing inside this sum, although they are not written out explicitly. In addition, we will designate the contributions from each of the four $c$-functions appearing in (4.94) by $\Xi_{4 \mathrm{~A}}$, $\Xi_{4 \mathrm{~B}}, \Xi_{4 \mathrm{C}}$, and $\Xi_{4 \mathrm{D}}$ respectively.

Consider $\Xi_{4 \mathrm{~A}}$. If we insert the definition (3.48), we get

$$
\begin{aligned}
\Xi_{4 \mathrm{~A}}=\sum_{d=1}^{n-1} \sum_{y=1}^{d} \sum_{z=d+1}^{n} & \frac{\left\langle y q^{\prime}\right\rangle\langle z q\rangle}{\langle q y\rangle\langle y z\rangle\left\langle q q^{\prime}\right\rangle} u^{\alpha}\left(k_{y}\right)[p+\kappa(1, y-1)]_{\alpha \dot{\alpha}} \\
& \times \frac{\left[\bar{\kappa}(z+1, n)+\bar{p}^{\prime}\right]^{\dot{\alpha} \beta} k_{z \beta \dot{\gamma}}\left[\bar{q}+\bar{q}^{\prime}+\bar{\kappa}(y, z)\right]^{\dot{\gamma} \gamma} u_{\gamma}(q)}{\left[q+q^{\prime}+\kappa(y, z-1)\right]^{2}\left[q+q^{\prime}+\kappa(y, z)\right]^{2}} .
\end{aligned}
$$


We now proceed to transform

$$
\mathcal{N}_{4 \mathrm{~A}} \equiv u^{\alpha}\left(k_{y}\right)[p+\kappa(1, y-1)]_{\alpha \dot{\alpha}}\left[\bar{\kappa}(z, n)+\bar{p}^{\prime}\right]^{\dot{\alpha} \beta} k_{z \beta \dot{\gamma}}\left[\bar{q}+\bar{q}^{\prime}+\bar{\kappa}(y, z)\right]^{\dot{\gamma} \gamma} u_{\gamma}(q)
$$

the numerator of (4.95), using the same tricks outlined in the discussion of $\Xi_{2}$ (cf. equations (4.48)-(4.53)). As in that case, our goal is to produce a series of terms containing inverse propagator factors in order to cancel as many denominators as possible. The result of this series of manipulations is

$$
\begin{aligned}
\mathcal{N}_{4 \mathrm{~A}}= & {\left[q+q^{\prime}+\kappa(y, z)\right]^{2} u^{\alpha}\left(k_{y}\right)[p+\kappa(1, y-1)]_{\alpha \dot{\alpha}}\left[\bar{\kappa}(z, n)+\bar{p}^{\prime}\right]^{\dot{\alpha} \beta} u_{\beta}(q) } \\
& -\left[q+q^{\prime}+\kappa(y, z-1)\right]^{2} u^{\alpha}\left(k_{y}\right)[p+\kappa(1, y-1)]_{\alpha \dot{\alpha}}\left[\bar{\kappa}(z+1, n)+\bar{p}^{\prime}\right]^{\dot{\alpha} \gamma} u_{\gamma}(q) \\
& +[p+\kappa(1, y-1)]^{2} u^{\alpha}\left(k_{y}\right)\left[q+q^{\prime}+\kappa(y, z-1)\right]_{\alpha \dot{\gamma}} \bar{k}_{z}^{\dot{\gamma} \gamma} u_{\gamma}(q) .
\end{aligned}
$$

Application of (4.97) to (4.95) yields

$$
\begin{aligned}
\Xi_{4 \mathrm{~A}}= & \sum_{d=1}^{n-1} \sum_{y=1}^{d} \sum_{z=d+1}^{n} \frac{\left\langle q^{\prime} y\right\rangle\langle q z\rangle}{\langle q y\rangle\langle y z\rangle\left\langle q q^{\prime}\right\rangle} \\
& \times\left[\frac{u^{\alpha}\left(k_{y}\right)[p+\kappa(1, y-1)]_{\alpha \dot{\alpha}}\left[\bar{\kappa}(z, n)+\bar{p}^{\prime}\right]^{\dot{\alpha} \beta} u_{\beta}(q)}{\left[q+q^{\prime}+\kappa(y, z-1)\right]^{2}}\right. \\
& \quad-\frac{u^{\alpha}\left(k_{y}\right)[p+\kappa(1, y-1)]_{\alpha \dot{\alpha}}\left[\bar{\kappa}(z+1, n)+\bar{p}^{\prime}\right]^{\dot{\alpha} \beta} u_{\beta}(q)}{\left[q+q^{\prime}+\kappa(y, z)\right]^{2}} \\
& \left.+[p+\kappa(1, y-1)]^{2} \frac{u^{\alpha}\left(k_{y}\right)\left[q+q^{\prime}+\kappa(y, z-1)\right]_{\alpha \dot{\gamma}} \bar{k}_{z}^{\dot{\gamma} \gamma} u_{\gamma}(q)}{\left[q+q^{\prime}+\kappa(y, z-1)\right]^{2}\left[q+q^{\prime}+\kappa(y, z)\right]^{2}}\right] .
\end{aligned}
$$

Finally, we write (A.14) in the form

$$
u^{\alpha}\left(k_{y}\right)\langle q z\rangle=u^{\alpha}\left(k_{z}\right)\langle q y\rangle+u^{\alpha}(q)\langle y z\rangle .
$$


to obtain

$$
\begin{aligned}
\Xi_{4 \mathrm{~A}}= & \sum_{d=1}^{n-1} \sum_{y=1}^{d} \sum_{z=d+1}^{n} \frac{\left\langle q^{\prime} y\right\rangle}{\langle y z\rangle\left\langle q q^{\prime}\right\rangle} \\
& \times\left[\frac{u^{\alpha}\left(k_{z}\right)[p+\kappa(1, y-1)]_{\alpha \dot{\alpha}}\left[\bar{\kappa}(z, n)+\bar{p}^{\prime}\right]^{\dot{\alpha} \beta} u_{\beta}(q)}{\left[q+q^{\prime}+\kappa(y, z-1)\right]^{2}}\right. \\
& \quad-\frac{u^{\alpha}\left(k_{z}\right)[p+\kappa(1, y-1)]_{\alpha \dot{\alpha}}\left[\bar{\kappa}(z+1, n)+\bar{p}^{\prime}\right]^{\dot{\alpha} \beta} u_{\beta}(q)}{\left[q+q^{\prime}+\kappa(y, z)\right]^{2}} \\
& \left.+[p+\kappa(1, y-1)]^{2} \frac{u^{\alpha}\left(k_{z}\right)\left[q+q^{\prime}+\kappa(y, z-1)\right]_{\alpha \dot{\gamma}} \bar{k}_{z}^{\dot{\gamma} \gamma} u_{\gamma}(q)}{\left[q+q^{\prime}+\kappa(y, z-1)\right]^{2}\left[q+q^{\prime}+\kappa(y, z)\right]^{2}}\right] \\
+ & \sum_{d=1}^{n-1} \sum_{y=1}^{d} \sum_{z=d+1}^{n} \frac{\left\langle q^{\prime} y\right\rangle}{\langle q y\rangle\left\langle q q^{\prime}\right\rangle} \\
& \times\left[\frac{u^{\alpha}(q)[p+\kappa(1, y-1)]_{\alpha \dot{\alpha}}\left[\bar{\kappa}(z, n)+\bar{p}^{\prime}\right]^{\dot{\alpha} \beta} u_{\beta}(q)}{\left[q+q^{\prime}+\kappa(y, z-1)\right]^{2}}\right. \\
& \quad-\frac{u^{\alpha}(q)[p+\kappa(1, y-1)]_{\alpha \dot{\alpha}}\left[\bar{\kappa}(z+1, n)+\bar{p}^{\prime}\right]^{\dot{\alpha} \beta} u_{\beta}(q)}{\left[q+q^{\prime}+\kappa(y, z)\right]^{2}} \\
& \left.+[p+\kappa(1, y-1)]^{2} \frac{u^{\alpha}(q)\left[q+q^{\prime}+\kappa(y, z-1)\right]_{\alpha \dot{\gamma}} \bar{k}_{z}^{\dot{\gamma} \gamma} u_{\gamma}(q)}{\left[q+q^{\prime}+\kappa(y, z-1)\right]^{2}\left[q+q^{\prime}+\kappa(y, z)\right]^{2}}\right] .
\end{aligned}
$$

We may use the same procedure to decompose $\Xi_{4 \mathrm{~B}}$. The result is

$$
\begin{aligned}
\Xi_{4 \mathrm{~B}}= & \sum_{d=1}^{n-1} \sum_{y=1}^{d} \sum_{z=d+1}^{n} \frac{\left\langle q^{\prime} y\right\rangle}{\langle y z\rangle\left\langle q q^{\prime}\right\rangle} \\
\times & {\left[\frac{u^{\alpha}\left(k_{z}\right)[p+\kappa(1, y)]_{\alpha \dot{\alpha}}\left[\bar{\kappa}(z+1, n)+\bar{p}^{\prime}\right]^{\dot{\alpha} \beta} u_{\beta}(q)}{\left[q+q^{\prime}+\kappa(y+1, z)\right]^{2}}\right.} \\
& \quad-\frac{u^{\alpha}\left(k_{z}\right)[p+\kappa(1, y)]_{\alpha \dot{\alpha}}\left[\bar{\kappa}(z, n)+\bar{p}^{\prime}\right]^{\dot{\alpha} \beta} u_{\beta}(q)}{\left[q+q^{\prime}+\kappa(y+1, z-1)\right]^{2}} \\
& \left.\quad-[p+\kappa(1, y)]^{2} \frac{u^{\alpha}\left(k_{z}\right)\left[q+q^{\prime}+\kappa(y+1, z-1)\right]_{\alpha \dot{\gamma}} \bar{k}_{z}^{\dot{\gamma} \gamma} u_{\gamma}(q)}{\left[q+q^{\prime}+\kappa(y+1, z-1)\right]^{2}\left[q+q^{\prime}+\kappa(y+1, z)\right]^{2}}\right] \\
+ & \sum_{d=1}^{n-1} \sum_{y=1}^{d} \sum_{z=d+1}^{n} \frac{\left\langle q^{\prime} y\right\rangle}{\langle q y\rangle\left\langle q q^{\prime}\right\rangle} \\
\times & {\left[\frac{u^{\alpha}(q)[p+\kappa(1, y)]_{\alpha \dot{\alpha}}\left[\bar{\kappa}(z+1, n)+\bar{p}^{\prime}\right]^{\dot{\alpha} \beta} u_{\beta}(q)}{\left[q+q^{\prime}+\kappa(y+1, z)\right]^{2}}\right.} \\
& \quad-\frac{u^{\alpha}(q)[p+\kappa(1, y)]_{\alpha \dot{\alpha}}\left[\bar{\kappa}(z, n)+\bar{p}^{\prime}\right]^{\dot{\alpha} \beta} u_{\beta}(q)}{\left[q+q^{\prime}+\kappa(y+1, z-1)\right]^{2}} \\
& \left.-[p+\kappa(1, y)]^{2} \frac{u^{\alpha}(q)\left[q+q^{\prime}+\kappa(y+1, z-1)\right]_{\alpha \dot{\gamma}} \bar{k}_{z}^{\dot{\gamma} \gamma} u_{\gamma}(q)}{\left[q+q^{\prime}+\kappa(y+1, z-1)\right]^{2}\left[q+q^{\prime}+\kappa(y+1, z)\right]^{2}}\right] .
\end{aligned}
$$


The remaining two contributions to $\Xi_{4}$ are processed in the same manner, but without the step corresponding to (4.99). Thus, we have

$$
\begin{aligned}
\Xi_{4 \mathrm{C}}= & \sum_{d=1}^{n-1} \sum_{y=1}^{d} \sum_{z=d+1}^{n} \frac{\left\langle q^{\prime} y\right\rangle}{\langle y z\rangle\left\langle q q^{\prime}\right\rangle} \\
\times & {\left[\frac{u^{\alpha}(q)[p+\kappa(1, y-1)]_{\alpha \dot{\alpha}}\left[\bar{\kappa}(z+1, n)+\bar{p}^{\prime}\right]^{\dot{\alpha} \beta} u_{\beta}\left(k_{z}\right)}{\left[q+q^{\prime}+\kappa(y, z)\right]^{2}}\right.} \\
& -\frac{u^{\alpha}(q)[p+\kappa(1, y)]_{\alpha \dot{\alpha}}\left[\bar{\kappa}(z+1, n)+\bar{p}^{\prime}\right]^{\dot{\alpha} \beta} u_{\beta}\left(k_{z}\right)}{\left[q+q^{\prime}+\kappa(y+1, z)\right]^{2}} \\
& \left.-\left[\kappa(z+1, n)+p^{\prime}\right]^{2} \frac{\left.u^{\alpha}(q) k_{y \alpha \dot{\gamma}}\left[\bar{q}+\bar{q}^{\prime}+\bar{\kappa}(y+1, z)\right]^{\dot{\gamma} \gamma} u_{\gamma}\left(k_{z}\right)\right]}{\left[q+q^{\prime}+\kappa(y+1, z)\right]^{2}\left[q+q^{\prime}+\kappa(y, z)\right]^{2}}\right] \\
\Xi_{4 \mathrm{D}}= & \sum_{d=1}^{n-1} \sum_{y=1}^{d} \sum_{z=d+1}^{n} \frac{\left\langle q^{\prime} y\right\rangle}{\langle y z\rangle\left\langle q q^{\prime}\right\rangle} \\
& \times\left[\frac{u^{\alpha}(q)[p+\kappa(1, y)]_{\alpha \dot{\alpha}}\left[\bar{\kappa}(z, n)+\bar{p}^{\prime}\right]^{\dot{\alpha} \beta} u_{\beta}\left(k_{z}\right)}{\left[q+q^{\prime}+\kappa(y+1, z-1)\right]^{2}}\right. \\
& \quad-\frac{u^{\alpha}(q)[p+\kappa(1, y-1)]_{\alpha \dot{\alpha}}\left[\bar{\kappa}(z, n)+\bar{p}^{\prime}\right]^{\dot{\alpha} \beta} u_{\beta}\left(k_{z}\right)}{\left[q+q^{\prime}+\kappa(y, z-1)\right]^{2}} \\
& \left.+\left[\kappa(z, n)+p^{\prime}\right]^{2} \frac{u^{\alpha}(q) k_{y \alpha \dot{\gamma}}\left[\bar{q}+\bar{q}^{\prime}+\bar{\kappa}^{\prime}(y+1, z-1)\right]^{\dot{\gamma} \gamma} u_{\gamma}\left(k_{z}\right)}{[q+\kappa(y+1, z-1)]^{2}\left[q+q^{\prime}+\kappa(y, z-1)\right]^{2}}\right] .
\end{aligned}
$$

The contributions tabulated in equations (4.100)-(4.103) consist of terms containing either one or two propagator factors. Let us begin with the latter type of terms. Denote by $\Xi_{4 a}$ the two double propagator terms containing $\frac{\left\langle q^{\prime} y\right\rangle}{\langle q y\rangle\left\langle q q^{\prime}\right\rangle}$ as a prefactor. That is,

$$
\begin{aligned}
\Xi_{4 a} \equiv & \sum_{d=1}^{n-1} \sum_{y=1}^{d} \sum_{z=d+1}^{n} \frac{\left\langle q^{\prime} y\right\rangle}{\langle q y\rangle\left\langle q q^{\prime}\right\rangle} \\
& \times\left[[p+\kappa(1, y-1)]^{2} \frac{u^{\alpha}(q)\left[q+q^{\prime}+\kappa(y, z-1)\right]_{\alpha \dot{\gamma}} \bar{k}_{z}^{\dot{\gamma} \gamma} u_{\gamma}(q)}{\left[q+q^{\prime}+\kappa(y, z-1)\right]^{2}\left[q+q^{\prime}+\kappa(y, z)\right]^{2}}\right. \\
& \left.\quad-[p+\kappa(1, y)]^{2} \frac{u^{\alpha}(q)\left[q+q^{\prime}+\kappa(y+1, z-1)\right]_{\alpha \dot{\gamma}} \bar{k}_{z}^{\dot{\gamma} \gamma} u_{\gamma}(q)}{\left[q+q^{\prime}+\kappa(y+1, z-1)\right]^{2}\left[q+q^{\prime}+\kappa(y+1, z)\right]^{2}}\right]
\end{aligned}
$$


Comparison with (3.48) shows that this is really

$$
\begin{aligned}
\Xi_{4 a}= & \sum_{d=1}^{n-1} \sum_{y=1}^{d} \sum_{z=d+1}^{n} \frac{\left\langle y q^{\prime}\right\rangle}{\left\langle y|q| q^{\prime}\right\rangle} \\
& \times\left[[p+\kappa(1, y-1)]^{2} c\left(q+q^{\prime}, y, y+1, \ldots, z\right)\right. \\
& \left.\quad-[p+\kappa(1, y)]^{2} c\left(q+q^{\prime}, y+1, y+2, \ldots, z\right)\right] .
\end{aligned}
$$

If we were to shift the sum over $y$ in the first term of (4.105) by one, both terms appearing in square brackets would be the same. Since the factor outside the square brackets also depends on $y$, we obtain instead

$$
\begin{aligned}
\Xi_{4 a}= & \sum_{d=1}^{n-1} \sum_{y=1}^{d-1} \sum_{z=d+1}^{n}\left[\frac{\left\langle y+1 q^{\prime}\right\rangle}{\left\langle y+1|q| q^{\prime}\right\rangle}-\frac{\left\langle y q^{\prime}\right\rangle}{\left\langle y|q| q^{\prime}\right\rangle}\right][p+\kappa(1, y)]^{2} c\left(q+q^{\prime}, y+1, y+2, \ldots, z\right) \\
& +\sum_{d=1}^{n-1} \sum_{z=d+1}^{n} \frac{\left\langle 1 q^{\prime}\right\rangle}{\left\langle 1|q| q^{\prime}\right\rangle} p^{2} c\left(q+q^{\prime}, 1, \ldots, z\right) \\
& -\sum_{d=1}^{n-1} \sum_{z=d+1}^{n} \frac{\left\langle d q^{\prime}\right\rangle}{\left\langle d|q| q^{\prime}\right\rangle}[p+\kappa(1, d)]^{2} c\left(q+q^{\prime}, d+1, \ldots, z\right) .
\end{aligned}
$$

The square brackets in the first contribution to (4.106) combine to form $-\frac{\langle y y+1\rangle}{\langle y|q| y+1\rangle}$ using (A.15). The second term vanishes, since $p^{2}=0$. The final term is precisely what is required to extend the first term to include $y=d$, the proper interpretation of $k_{d+1}$ in this context being $q^{\prime}$. Thus,

$$
\Xi_{4 a}=-\sum_{d=1}^{n-1} \sum_{y=1}^{d} \sum_{z=d+1}^{n} \frac{\langle y y+1\rangle}{\langle y|q| y+1\rangle}[p+\kappa(1, y)]^{2} c\left(q+q^{\prime}, y+1, y+2, \ldots, z\right) .
$$

Note that we may extend the sums to include $d=y=0$ since such a term is proportional to $p^{2}=0$. Thus, the contribution to the amplitude from (4.107) may be written

$$
\begin{aligned}
\mathcal{M}_{4 a}=\frac{i}{2}( & -g \sqrt{2})^{n+2} \sum_{\mathcal{P}(1 \ldots n)} \sum_{d=0}^{n-1} \sum_{y=0}^{d} \sum_{z=d+1}^{n}(\Omega[1, d])^{i}{ }_{\ell}(\Omega[d+1, n])_{k}{ }^{j} \\
& \times \frac{\langle p q\rangle^{2}[p+\kappa(1, y)]^{2} c\left(q+q^{\prime}, y+1, \ldots, z\right)}{\langle p|1, \ldots, y| q\rangle\left\langle q|y+1, \ldots, d| q^{\prime}\right\rangle\left\langle q|d+1, \ldots, n| p^{\prime}\right\rangle} .
\end{aligned}
$$


The contribution represented by (4.108) identically cancels the last term appearing in $\mathcal{M}_{2}$ (see equation (4.56)).

Next, we turn to the double propagator contributions in (4.100)-(4.101) which have $\frac{\left\langle q^{\prime} y\right\rangle}{\langle y z\rangle\left\langle q q^{\prime}\right\rangle}$ as a prefactor. These two terms are

$$
\begin{aligned}
\Xi_{4 b} \equiv & \sum_{d=1}^{n-1} \sum_{y=1}^{d} \sum_{z=d+1}^{n} \frac{\left\langle q^{\prime} y\right\rangle}{\langle y z\rangle\left\langle q q^{\prime}\right\rangle} \\
\times & {\left[[p+\kappa(1, y-1)]^{2} \frac{u^{\alpha}\left(k_{z}\right)\left[q+q^{\prime}+\kappa(y, z-1)\right]_{\alpha \dot{\gamma}} \bar{k}_{z}^{\dot{\gamma} \gamma} u_{\gamma}(q)}{\left[q+q^{\prime}+\kappa(y, z-1)\right]^{2}\left[q+q^{\prime}+\kappa(y, z)\right]^{2}}\right.} \\
& \left.\quad-[p+\kappa(1, y)]^{2} \frac{u^{\alpha}\left(k_{z}\right)\left[q+q^{\prime}+\kappa(y+1, z-1)\right]_{\alpha \dot{\gamma}} \bar{k}_{z}^{\dot{\gamma} \gamma} u_{\gamma}(q)}{\left[q+q^{\prime}+\kappa(y+1, z-1)\right]^{2}\left[q+q^{\prime}+\kappa(y+1, z)\right]^{2}}\right] .
\end{aligned}
$$

Because of the relations (A.11) and (A.21a), we may write

$$
\begin{aligned}
u^{\alpha}\left(k_{z}\right) & {\left[q+q^{\prime}+\kappa(y, z-1)\right]_{\alpha \dot{\gamma}} \bar{k}_{z}^{\dot{\gamma} \gamma} u_{\gamma}(q)=} \\
& =2 k_{z} \cdot\left[q+q^{\prime}+\kappa(y, z-1)\right]\langle z q\rangle \\
& =\left\{\left[q+q^{\prime}+\kappa(y, z)\right]^{2}-\left[q+q^{\prime}+\kappa(y, z-1)\right]^{2}\right\}\langle z q\rangle .
\end{aligned}
$$

This produces

$$
\begin{aligned}
\Xi_{4 b}= & \sum_{d=1}^{n-1} \sum_{y=1}^{d} \sum_{z=d+1}^{n} \frac{\left\langle q^{\prime} y\right\rangle\langle z q\rangle}{\langle y z\rangle\left\langle q q^{\prime}\right\rangle} \\
& \times\left[-\frac{[p+\kappa(1, y-1)]^{2}}{\left[q+q^{\prime}+\kappa(y, z)\right]^{2}}+\frac{[p+\kappa(1, y-1)]^{2}}{\left[q+q^{\prime}+\kappa(y, z-1)\right]^{2}}\right. \\
& \left.+\frac{[p+\kappa(1, y)]^{2}}{\left[q+q^{\prime}+\kappa(y+1, z)\right]^{2}}-\frac{[p+\kappa(1, y)]^{2}}{\left[q+q^{\prime}+\kappa(y+1, z-1)\right]^{2}}\right] .
\end{aligned}
$$

We set (4.111) aside for later cancellation.

There are two double propagator terms not yet accounted for. These appear in equations (4.102) and (4.103). They are

$$
\begin{aligned}
\Xi_{4 c d} \equiv & \sum_{d=1}^{n-1} \sum_{y=1}^{d} \sum_{z=d+1}^{n} \frac{\left\langle q^{\prime} y\right\rangle}{\langle y z\rangle\left\langle q q^{\prime}\right\rangle} \\
& \times\left[\left[\kappa(z, n)+p^{\prime}\right]^{2} \frac{\langle q y\rangle \bar{u}_{\dot{\gamma}}\left(k_{y}\right)\left[\bar{q}+\bar{q}^{\prime}+\bar{\kappa}(y+1, z-1)\right]^{\dot{\gamma} \gamma} u_{\gamma}\left(k_{z}\right)}{\left[q+q^{\prime}+\kappa(y+1, z-1)\right]^{2}\left[q+q^{\prime}+\kappa(y, z-1)\right]^{2}}\right. \\
& \left.\quad-\left[\kappa(z+1, n)+p^{\prime}\right]^{2} \frac{\langle q y\rangle \bar{u}_{\dot{\gamma}}\left(k_{y}\right)\left[\bar{q}+\bar{q}^{\prime}+\bar{\kappa}(y+1, z)\right]^{\dot{\gamma} \gamma} u_{\gamma}\left(k_{z}\right)}{\left[q+q^{\prime}+\kappa(y+1, z)\right]^{2}\left[q+q^{\prime}+\kappa(y, z)\right]^{2}}\right] .
\end{aligned}
$$


We break (4.112) into two pieces by applying (A.14), written as

$$
u_{\gamma}\left(k_{z}\right)\langle q y\rangle=-u_{\gamma}\left(k_{y}\right)\langle z q\rangle-u_{\gamma}(q)\langle y z\rangle \text {. }
$$

Call the piece of (4.112) corresponding to the first term of (4.113) $\Xi_{4 c}$ and the remainder $\Xi_{4 d}$.

For $\Xi_{4 c}$ we have

$$
\begin{aligned}
\Xi_{4 c}= & \sum_{d=1}^{n-1} \sum_{y=1}^{d} \sum_{z=d+1}^{n} \frac{\left\langle q^{\prime} y\right\rangle}{\langle y z\rangle\left\langle q q^{\prime}\right\rangle} \\
& \times\left[-\left[\kappa(z, n)+p^{\prime}\right]^{2} \frac{\langle z q\rangle \bar{u}_{\dot{\gamma}}\left(k_{y}\right)\left[\bar{q}+\bar{q}^{\prime}+\bar{\kappa}(y+1, z-1)\right]^{\dot{\gamma} \gamma} u_{\gamma}\left(k_{y}\right)}{\left[q+q^{\prime}+\kappa(y+1, z-1)\right]^{2}\left[q+q^{\prime}+\kappa(y, z-1)\right]^{2}}\right. \\
& \left.+\left[\kappa(z+1, n)+p^{\prime}\right]^{2} \frac{\langle z q\rangle \bar{u}_{\dot{\gamma}}\left(k_{y}\right)\left[\bar{q}+\bar{q}^{\prime}+\bar{\kappa}(y+1, z)\right]^{\dot{\gamma} \gamma} u_{\gamma}\left(k_{y}\right)}{\left[q+q^{\prime}+\kappa(y+1, z)\right]^{2}\left[q+q^{\prime}+\kappa(y, z)\right]^{2}}\right] .
\end{aligned}
$$

But, we may write

$$
\begin{aligned}
\bar{u}_{\dot{\gamma}}\left(k_{y}\right) & {\left[\bar{q}+\bar{q}^{\prime}+\bar{\kappa}(y+1, z-1)\right]^{\dot{\gamma} \gamma} u_{\gamma}\left(k_{y}\right)=} \\
& =2 k_{y} \cdot\left[q+q^{\prime}+\kappa(y+1, z-1)\right] \\
& =\left[q+q^{\prime}+\kappa(y, z-1)\right]^{2}-\left[q+q^{\prime}+\kappa(y+1, z-1)\right]^{2},
\end{aligned}
$$

giving us

$$
\begin{aligned}
\Xi_{4 c}= & \sum_{d=1}^{n-1} \sum_{y=1}^{d} \sum_{z=d+1}^{n} \frac{\left\langle q^{\prime} y\right\rangle\langle z q\rangle}{\langle y z\rangle\left\langle q q^{\prime}\right\rangle} \\
& \times\left[-\frac{\left[\kappa(z, n)+p^{\prime}\right]^{2}}{\left[q+q^{\prime}+\kappa(y+1, z-1)\right]^{2}}+\frac{\left[\kappa(z, n)+p^{\prime}\right]^{2}}{\left[q+q^{\prime}+\kappa(y, z-1)\right]^{2}}\right. \\
& \left.\quad+\frac{\left[\kappa(z+1, n)+p^{\prime}\right]^{2}}{\left[q+q^{\prime}+\kappa(y+1, z)\right]^{2}}-\frac{\left[\kappa(z+1, n)+p^{\prime}\right]^{2}}{\left[q+q^{\prime}+\kappa(y, z)\right]^{2}}\right] .
\end{aligned}
$$

This is another contribution which will be cancelled later.

The other piece of (4.112) is

$$
\begin{aligned}
\Xi_{4 d} \equiv & \sum_{d=1}^{n-1} \sum_{y=1}^{d} \sum_{z=d+1}^{n} \frac{\left\langle q^{\prime} y\right\rangle}{\left\langle q q^{\prime}\right\rangle} \\
& \times\left[-\left[\kappa(z, n)+p^{\prime}\right]^{2} \frac{\bar{u}_{\dot{\gamma}}\left(k_{y}\right)\left[\bar{q}+\bar{q}^{\prime}+\bar{\kappa}(y+1, z-1)\right]^{\dot{\gamma} \gamma} u_{\gamma}(q)}{\left[q+q^{\prime}+\kappa(y+1, z-1)\right]^{2}\left[q+q^{\prime}+\kappa(y, z-1)\right]^{2}}\right. \\
& \left.\quad+\left[\kappa(z+1, n)+p^{\prime}\right]^{2} \frac{\bar{u}_{\dot{\gamma}}\left(k_{y}\right)\left[\bar{q}+\bar{q}^{\prime}+\bar{\kappa}(y+1, z)\right]^{\dot{\gamma} \gamma} u_{\gamma}(q)}{\left[q+q^{\prime}+\kappa(y+1, z)\right]^{2}\left[q+q^{\prime}+\kappa(y, z)\right]^{2}}\right] .
\end{aligned}
$$


Since the two terms in the square brackets differ by 1 unit in $z$, and there is no other $z$-dependence, we may do the sum on $z$, the result being only the endpoints:

$$
\begin{aligned}
\Xi_{4 d}=\sum_{d=1}^{n-1} \sum_{y=1}^{d} \frac{\left\langle q^{\prime} y\right\rangle}{\left\langle q q^{\prime}\right\rangle}\left[-\left[\kappa(d+1, n)+p^{\prime}\right]^{2} \frac{\bar{u}_{\dot{\gamma}}\left(k_{y}\right)\left[\bar{q}+\bar{q}^{\prime}+\bar{\kappa}(y+1, d)\right]^{\dot{\gamma} \gamma} u_{\gamma}(q)}{\left[q+q^{\prime}+\kappa(y+1, d)\right]^{2}\left[q+q^{\prime}+\kappa(y, d)\right]^{2}}\right. \\
\left.+p^{\prime 2} \frac{\bar{u}_{\dot{\gamma}}\left(k_{y}\right)\left[\bar{q}+\bar{q}^{\prime}+\bar{\kappa}(y+1, n)\right]^{\dot{\gamma} \gamma} u_{\gamma}(q)}{\left[q+q^{\prime}+\kappa(y+1, n)\right]^{2}\left[q+q^{\prime}+\kappa(y, n)\right]^{2}}\right] .
\end{aligned}
$$

Since $p^{2}=0$, the second contribution to (4.118) vanishes. In addition, note that we may add $d=n$ to the sum appearing in the first contribution with no penalty since such a term is also proportional to $p^{2}$. Thus

$$
\Xi_{4 d}=\sum_{d=1}^{n} \sum_{y=1}^{d} \frac{\left\langle q^{\prime} y\right\rangle}{\left\langle q q^{\prime}\right\rangle\langle q y\rangle}\left[\kappa(d+1, n)+p^{\prime}\right]^{2} c\left(q+q^{\prime}, d, d-1, \ldots, y\right),
$$

where we have multiplied by $\frac{\langle q y\rangle}{\langle q y\rangle}$ and used (3.48) to identify the factor of $c$. The contribution to $\mathcal{M}_{4}$ that corresponds to (4.119) is

$$
\begin{aligned}
\mathcal{M}_{4 d}= & \frac{-i}{2}(-g \sqrt{2})^{n+2} \sum_{\mathcal{P}(1 \ldots n)} \sum_{d=1}^{n} \sum_{y=1}^{d}(\Omega[1, d])^{i}{ }_{\ell}(\Omega[d+1, n])_{k}^{j} \\
& \times \frac{\left\langle q^{\prime} y\right\rangle}{\left\langle q q^{\prime}\right\rangle\langle q y\rangle} \frac{\langle p q\rangle^{2}\left[\kappa(d+1, n)+p^{\prime}\right]^{2} c\left(q+q^{\prime}, d, d-1, \ldots, y\right)}{\left\langle p|1, \ldots, d| q^{\prime}\right\rangle\left\langle q|d+1, \ldots, n| p^{\prime}\right\rangle}
\end{aligned}
$$

which, exactly cancels the contribution from $\mathcal{M}_{3 \mathrm{~A}}$ given in equation (4.66) (recall that the spinor inner product is antisymmetric, $\left.\left\langle y q^{\prime}\right\rangle=-\left\langle q^{\prime} y\right\rangle\right)$.

Having finished with the terms containing two propagators, we turn to the remaining contributions with just one propagator. Let us begin with the terms proportional to $\frac{\left\langle q^{\prime} y\right\rangle}{\langle q y\rangle\left\langle q q^{\prime}\right\rangle}$. These contributions come from (4.100) and (4.101) 
and comprise the following four terms:

$$
\begin{aligned}
\Xi_{4 e} \equiv & \sum_{d=1}^{n-1} \sum_{y=1}^{d} \sum_{z=d+1}^{n} \frac{\left\langle q^{\prime} y\right\rangle}{\langle q y\rangle\left\langle q q^{\prime}\right\rangle} \\
& \times\left[\frac{u^{\alpha}(q)[p+\kappa(1, y-1)]_{\alpha \dot{\alpha}}\left[\bar{\kappa}(z, n)+\bar{p}^{\prime}\right]^{\dot{\alpha} \beta} u_{\beta}(q)}{\left[q+q^{\prime}+\kappa(y, z-1)\right]^{2}}\right. \\
& -\frac{u^{\alpha}(q)[p+\kappa(1, y-1)]_{\alpha \dot{\alpha}}\left[\bar{\kappa}(z+1, n)+\bar{p}^{\prime}\right]^{\dot{\alpha} \beta} u_{\beta}(q)}{\left[q+q^{\prime}+\kappa(y, z)\right]^{2}} \\
& +\frac{u^{\alpha}(q)[p+\kappa(1, y)]_{\alpha \dot{\alpha}}\left[\bar{\kappa}(z+1, n)+\bar{p}^{\prime}\right]^{\dot{\alpha} \beta} u_{\beta}(q)}{\left[q+q^{\prime}+\kappa(y+1, z)\right]^{2}} \\
& \left.-\frac{u^{\alpha}(q)[p+\kappa(1, y)]_{\alpha \dot{\alpha}}\left[\bar{\kappa}(z, n)+\bar{p}^{\prime}\right]^{\dot{\alpha} \beta} u_{\beta}(q)}{\left[q+q^{\prime}+\kappa(y+1, z-1)\right]^{2}}\right] .
\end{aligned}
$$

The sum on $z$ may be performed in (4.121) with the contributions from the interior points in the summation cancelling in pairs, leaving just

$$
\begin{aligned}
\Xi_{4 e}= & \sum_{d=1}^{n} \sum_{y=1}^{d} \frac{\left\langle q^{\prime} y\right\rangle}{\langle q y\rangle\left\langle q q^{\prime}\right\rangle} \\
\times & \frac{u^{\alpha}(q)[p+\kappa(1, y-1)]_{\alpha \dot{\alpha}}\left[\bar{\kappa}(d+1, n)+\bar{p}^{\prime}\right]^{\dot{\alpha} \beta} u_{\beta}(q)}{\left[q+q^{\prime}+\kappa(y, d)\right]^{2}} \\
& \quad-\frac{u^{\alpha}(q)[p+\kappa(1, y-1)]_{\alpha \dot{\alpha}} \bar{p}^{\prime \dot{\alpha} \beta} u_{\beta}(q)}{\left[q+q^{\prime}+\kappa(y, n)\right]^{2}} \\
& +\frac{u^{\alpha}(q)[p+\kappa(1, y)]_{\alpha \dot{\alpha}} \bar{p}^{\prime \dot{\alpha} \beta} u_{\beta}(q)}{\left[q+q^{\prime}+\kappa(y+1, n)\right]^{2}} \\
& \left.\quad-\frac{u^{\alpha}(q)[p+\kappa(1, y)]_{\alpha \dot{\alpha}}\left[\bar{\kappa}(d+1, n)+\bar{p}^{\prime}\right]^{\dot{\alpha} \beta} u_{\beta}(q)}{\left[q+q^{\prime}+\kappa(y+1, d)\right]^{2}}\right] .
\end{aligned}
$$

We have added the term $d=n$ to (4.122) since the quantity in brackets vanishes for that value of $d$.

At this point, we see that there are pairs of terms in the square brackets that differ by one unit in $y$. However, the prefactor is not independent of $y$. Following the same procedure used in the discussion of $\Xi_{3 \mathrm{~B}}(c f$. equations (4.72)- 
(4.77)), we obtain

$$
\begin{aligned}
\Xi_{4 e}= & \sum_{d=1}^{n} \sum_{y=1}^{d} \frac{\langle y y+1\rangle}{\langle y|q| y+1\rangle} \frac{u^{\alpha}(q)[p+\kappa(1, y)]_{\alpha \dot{\alpha}} \bar{p}^{\dot{\alpha} \beta} u_{\beta}(q)}{\left[p+p^{\prime}+\kappa(1, y)\right]^{2}} \\
& -\sum_{d=1}^{n} \frac{\left\langle 1 q^{\prime}\right\rangle}{\left\langle 1|q| q^{\prime}\right\rangle} \frac{u^{\alpha}(q) p_{\alpha \dot{\alpha}} \bar{p}^{\prime \dot{\alpha} \beta} u_{\beta}(q)}{\left[p+p^{\prime}\right]^{2}} \\
& -\sum_{d=1}^{n} \sum_{y=1} \frac{\langle y y+1\rangle}{\langle y|q| y+1\rangle} \frac{u^{\alpha}(q)[p+\kappa(1, y)]_{\alpha \dot{\alpha}}\left[\bar{\kappa}(d+1, n)+\bar{p}^{\prime}\right]^{\dot{\alpha} \beta} u_{\beta}(q)}{\left[q+q^{\prime}+\kappa(y+1, d)\right]^{2}} \\
& +\sum_{d=1}^{n} \frac{\left\langle 1 q^{\prime}\right\rangle}{\left\langle 1|q| q^{\prime}\right\rangle} \frac{u^{\alpha}(q) p_{\alpha \dot{\alpha}}\left[\bar{\kappa}(d+1, n)+\bar{p}^{\prime}\right]^{\dot{\alpha} \beta} u_{\beta}(q)}{\left[q+q^{\prime}+\kappa(1, d)\right]^{2}} .
\end{aligned}
$$

The contribution to $\mathcal{M}_{4}$ from (4.123) is

$$
\begin{aligned}
& \mathcal{M}_{4 e}=\frac{-i}{2}(-g \sqrt{2})^{n+2} \sum_{\mathcal{P}(1 \ldots n)} \sum_{d=1}^{n} \sum_{y=1}^{d}(\Omega[1, d])^{i}{ }_{\ell}(\Omega[d+1, n])_{k}{ }^{j} \\
& \times \frac{\langle p q\rangle^{2} u^{\alpha}(q)[p+\kappa(1, y)]_{\alpha \dot{\alpha}} \bar{p}^{\prime \dot{\alpha} \beta} u_{\beta}(q)}{\langle p|1, \ldots, y| q\rangle\left\langle q|y+1, \ldots, d| q^{\prime}\right\rangle\left\langle q|d+1, \ldots, n| p^{\prime}\right\rangle} \\
& \times \frac{1}{\left[p+p^{\prime}+\kappa(1, y)\right]^{2}} \\
& +\frac{i}{2}(-g \sqrt{2})^{n+2} \sum_{\mathcal{P}(1 \ldots n)} \sum_{d=1}^{n}(\Omega[1, d])_{\ell}^{i}(\Omega[d+1, n])_{k}^{j} \\
& \times \frac{\left\langle 1 q^{\prime}\right\rangle}{\left\langle 1|q| q^{\prime}\right\rangle} \frac{\langle p q\rangle^{2} u^{\alpha}(q) p_{\alpha \dot{\alpha}} \bar{p}^{\dot{\alpha} \beta} u_{\beta}(q)}{\left[p+p^{\prime}\right]^{2}\left\langle p|1, \ldots, d| q^{\prime}\right\rangle\left\langle q|d+1, \ldots, n| p^{\prime}\right\rangle} \\
& +\frac{i}{2}(-g \sqrt{2})^{n+2} \sum_{\mathcal{P}(1 \ldots n)} \sum_{d=1}^{n} \sum_{y=1}^{d}(\Omega[1, d])_{\ell}^{i}(\Omega[d+1, n])_{k}^{j} \\
& \times \frac{\langle p q\rangle^{2} u^{\alpha}(q)[p+\kappa(1, y)]_{\alpha \dot{\alpha}}\left[\bar{\kappa}(d+1, n)+\bar{p}^{\prime}\right]^{\dot{\alpha} \beta} u_{\beta}(q)}{\langle p|1, \ldots, y| q\rangle\left\langle q|y+1, \ldots, d| q^{\prime}\right\rangle\left\langle q|d+1, \ldots, n| p^{\prime}\right\rangle} \\
& \times \frac{1}{\left[q+q^{\prime}+\kappa(y+1, d)\right]^{2}} \\
& -\frac{i}{2}(-g \sqrt{2})^{n+2} \sum_{\mathcal{P}(1 \ldots n)} \sum_{d=1}^{n}(\Omega[1, d])_{\ell}^{i}(\Omega[d+1, n])_{k}^{j} \\
& \times \frac{\left\langle 1 q^{\prime}\right\rangle}{\left\langle 1|q| q^{\prime}\right\rangle} \frac{\langle p q\rangle^{2} u^{\alpha}(q) p_{\alpha \dot{\alpha}}\left[\bar{\kappa}(d+1, n)+\bar{p}^{\prime}\right]^{\dot{\alpha} \beta} u_{\beta}(q)}{\left\langle p|1, \ldots, d| q^{\prime}\right\rangle\left\langle q|d+1, \ldots, n| p^{\prime}\right\rangle} \\
& \times \frac{1}{\left[q+q^{\prime}+\kappa(1, d)\right]^{2}} \text {. }
\end{aligned}
$$


The third and fourth terms of $(4.124)$ cancel $\mathcal{M}_{3 \mathrm{~B}}$ in its entirety (see equation (4.79)). The first two terms of (4.124) have no compensating contributions.

We collect the remaining eight single propagator contributions from equations (4.100)-(4.103) and form

$$
\begin{aligned}
\Xi_{4 f}=\sum_{d=1}^{n-1} \sum_{y=1}^{d} & \sum_{z=d+1}^{n} \frac{\left\langle q^{\prime} y\right\rangle}{\langle y z\rangle\left\langle q q^{\prime}\right\rangle}\left[\frac{u^{\alpha}\left(k_{z}\right)[p+\kappa(1, y-1)]_{\alpha \dot{\alpha}}\left[\bar{\kappa}(z, n)+\bar{p}^{\prime}\right]^{\dot{\alpha} \beta} u_{\beta}(q)}{\left[q+q^{\prime}+\kappa(y, z-1)\right]^{2}}\right. \\
& -\frac{u^{\alpha}\left(k_{z}\right)[p+\kappa(1, y-1)]_{\alpha \dot{\alpha}}\left[\bar{\kappa}(z+1, n)+\bar{p}^{\prime}\right]^{\dot{\alpha} \beta} u_{\beta}(q)}{\left[q+q^{\prime}+\kappa(y, z)\right]^{2}} \\
& +\frac{u^{\alpha}\left(k_{z}\right)[p+\kappa(1, y)]_{\alpha \dot{\alpha}}\left[\bar{\kappa}(z+1, n)+\bar{p}^{\prime}\right]^{\dot{\alpha} \beta} u_{\beta}(q)}{\left[q+q^{\prime}+\kappa(y+1, z)\right]^{2}} \\
& -\frac{u^{\alpha}\left(k_{z}\right)[p+\kappa(1, y)]_{\alpha \dot{\alpha}}\left[\bar{\kappa}(z, n)+\bar{p}^{\prime}\right]^{\dot{\alpha} \beta} u_{\beta}(q)}{\left[q+q^{\prime}+\kappa(y+1, z-1)\right]^{2}} \\
& +\frac{u^{\alpha}(q)[p+\kappa(1, y)]_{\alpha \dot{\alpha}}\left[\bar{\kappa}(z, n)+\bar{p}^{\prime}\right]^{\dot{\alpha} \beta} u_{\beta}\left(k_{z}\right)}{\left[q+q^{\prime}+\kappa(y+1, z-1)\right]^{2}} \\
& -\frac{u^{\alpha}(q)[p+\kappa(1, y-1)]_{\alpha \dot{\alpha}}\left[\bar{\kappa}(z, n)+\bar{p}^{\prime}\right]^{\dot{\alpha} \beta} u_{\beta}\left(k_{z}\right)}{\left[q+q^{\prime}+\kappa(y, z-1)\right]^{2}} \\
& +\frac{u^{\alpha}(q)[p+\kappa(1, y-1)]_{\alpha \dot{\alpha}}\left[\bar{\kappa}(z+1, n)+\bar{p}^{\prime}\right]^{\dot{\alpha} \beta} u_{\beta}\left(k_{z}\right)}{\left[q+q^{\prime}+\kappa(y, z)\right]^{2}} \\
& -\frac{u^{\alpha}(q)[p+\kappa(1, y)]_{\alpha \dot{\alpha}}\left[\bar{\kappa}(z+1, n)+\bar{p}^{\prime}\right]^{\dot{\alpha} \beta} u_{\beta}\left(k_{z}\right)}{\left[q+q^{\prime}+\kappa(y+1, z)\right]^{2}} .
\end{aligned}
$$

The terms in (4.125) occur in pairs with common denominators. Note that in

every pair, we have the form

$$
\begin{aligned}
u^{\alpha}\left(k_{z}\right) X_{\alpha \dot{\alpha}} \bar{Y}^{\dot{\alpha} \beta} & u_{\beta}(q)-u^{\alpha}(q) X_{\alpha \dot{\alpha}} \bar{Y}^{\dot{\alpha} \beta} u_{\beta}\left(k_{z}\right)= \\
& =u^{\alpha}\left(k_{z}\right)\left[X_{\alpha \dot{\alpha}} \bar{Y}^{\dot{\alpha} \beta}+Y_{\alpha \dot{\alpha}} \bar{X}^{\dot{\alpha} \beta}\right] u_{\beta}(q) \\
& =2 X \cdot Y\langle z q\rangle,
\end{aligned}
$$


where $X$ and $Y$ are momenta and we have applied (A.3). If we use (4.126) on each of the four pairs in (4.125), we obtain

$$
\begin{aligned}
\Xi_{4 f}= & \sum_{d=1}^{n-1} \sum_{y=1}^{d} \sum_{z=d+1}^{n} \frac{\left\langle q^{\prime} y\right\rangle\langle z q\rangle}{\langle y z\rangle\left\langle q q^{\prime}\right\rangle}\left[\frac{2[p+\kappa(1, y-1)] \cdot\left[\kappa(z, n)+p^{\prime}\right]}{\left[q+q^{\prime}+\kappa(y, z-1)\right]^{2}}\right. \\
& -\frac{2[p+\kappa(1, y-1)] \cdot\left[\kappa(z+1, n)+p^{\prime}\right]}{\left[q+q^{\prime}+\kappa(y, z)\right]^{2}}+\frac{2[p+\kappa(1, y)] \cdot\left[\kappa(z+1, n)+p^{\prime}\right]}{\left[q+q^{\prime}+\kappa(y+1, z)\right]^{2}} \\
& \left.-\frac{2[p+\kappa(1, y)] \cdot\left[\kappa(z, n)+p^{\prime}\right]}{\left[q+q^{\prime}+\kappa(y+1, z-1)\right]^{2}}\right] .
\end{aligned}
$$

Each of the four terms in (4.127) may be treated using momentum conservation.

For example, momentum conservation tells us that

$$
\begin{aligned}
{\left[q+q^{\prime}+\kappa(y, z-1)\right]^{2}=} & {\left[p+\kappa(1, y-1)+\kappa(z, n)+p^{\prime}\right]^{2} } \\
= & {[p+\kappa(1, y-1)]^{2}+\left[\kappa(z, n)+p^{\prime}\right]^{2} } \\
& +2[p+\kappa(1, y-1)] \cdot\left[\kappa(z, n)+p^{\prime}\right] .
\end{aligned}
$$

When we apply (4.128) to (4.127), the result is

$$
\begin{aligned}
\Xi_{4 f}=\sum_{d=1}^{n-1} \sum_{y=1}^{d} \sum_{z=d+1}^{n} \frac{\left\langle q^{\prime} y\right\rangle\langle z q\rangle}{\langle y z\rangle\left\langle q q^{\prime}\right\rangle} \\
\quad \times\left[\quad 1-\frac{[p+\kappa(1, y-1)]^{2}}{\left[q+q^{\prime}+\kappa(y, z-1)\right]^{2}}-\frac{\left[\kappa(z, n)+p^{\prime}\right]^{2}}{\left[q+q^{\prime}+\kappa(y, z-1)\right]^{2}}\right. \\
\quad-1+\frac{[p+\kappa(1, y-1)]^{2}}{\left[q+q^{\prime}+\kappa(y, z)\right]^{2}}+\frac{\left[\kappa(z+1, n)+p^{\prime}\right]^{2}}{\left[q+q^{\prime}+\kappa(y, z)\right]^{2}} \\
\quad+1-\frac{[p+\kappa(1, y)]^{2}}{\left[q+q^{\prime}+\kappa(y+1, z)\right]^{2}}-\frac{\left[\kappa(z+1, n)+p^{\prime}\right]^{2}}{\left[q+q^{\prime}+\kappa(y+1, z)\right]^{2}} \\
\left.\quad-1+\frac{[p+\kappa(1, y)]^{2}}{\left[q+q^{\prime}+\kappa(y+1, z-1)\right]^{2}}+\frac{\left[\kappa(z, n)+p^{\prime}\right]^{2}}{\left[q+q^{\prime}+\kappa(y+1, z-1)\right]^{2}}\right] .
\end{aligned}
$$

The 1's cancel among themselves. The remaining terms dispose of the contributions from $\Xi_{4 b}$ and $\Xi_{4 c}$ (equations (4.111) and (4.116) respectively).

\subsection{Final considerations}

We now collect the surviving terms and combine them into the amplitude for the process (1.1). These terms are: the second contribution to $\mathcal{M}_{2}$ (equation 
(4.56)), the first two terms of $\mathcal{M}_{4 e}$ (equation (4.124)), and, of course, the $\frac{1}{N}$ contribution $\mathcal{M}_{5}$ (equation (4.29)). Let us start by simplifying the remaining non- $\frac{1}{N}$ pieces, which read

$$
\begin{aligned}
\mathcal{M}_{T} \equiv & \frac{i}{2}(-g \sqrt{2})^{n+2} \sum_{\mathcal{P}(1 \ldots n)} \sum_{d=0}^{n} \sum_{b=0}^{d}(\Omega[1, d])_{\ell}^{i}(\Omega[d+1, n])_{k}{ }^{j} \\
& \times \frac{\langle p q\rangle^{2} u^{\alpha}(q)[p+\kappa(1, b)]_{\alpha \dot{\beta}} \bar{p}^{\prime \dot{\beta} \gamma} u_{\gamma}(q)}{\langle p|1, \ldots, b| q\rangle\left\langle q|b+1, \ldots, d| q^{\prime}\right\rangle\left\langle q|d+1, \ldots, n| p^{\prime}\right\rangle} \\
& \times \frac{1}{\left[p+p^{\prime}+\kappa(1, b)\right]^{2}} \\
- & \frac{i}{2}(-g \sqrt{2})^{n+2} \sum_{\mathcal{P}(1 \ldots n)} \sum_{d=1}^{n} \sum_{y=1}^{d}(\Omega[1, d])^{i} \ell^{i}(\Omega[d+1, n])_{k}{ }^{j} \\
& \times \frac{\langle p q\rangle^{2} u^{\alpha}(q)[p+\kappa(1, y)]_{\alpha \dot{\alpha}} \bar{p}^{\prime \dot{\alpha} \beta} u_{\beta}(q)}{\langle p|1, \ldots, y| q\rangle\left\langle q|y+1, \ldots, d| q^{\prime}\right\rangle\left\langle q|d+1, \ldots, n| p^{\prime}\right\rangle} \\
& \times \frac{1}{\left[p+p^{\prime}+\kappa(1, y)\right]^{2}} \\
+ & \frac{i}{2}(-g \sqrt{2})^{n+2} \sum_{\mathcal{P}(1 \ldots n)} \sum_{d=1}^{n}(\Omega[1, d])^{i}{ }_{\ell}(\Omega[d+1, n])_{k}{ }^{j} \\
& \times \frac{\left\langle 1 q^{\prime}\right\rangle}{\left\langle 1|q| q^{\prime}\right\rangle} \frac{\langle p q\rangle^{2} u^{\alpha}(q) p_{\alpha \dot{\alpha}} \bar{p}^{\dot{\alpha} \beta} u_{\beta}(q)}{\left[p+p^{\prime}\right]^{2}\left\langle p|1, \ldots, d| q^{\prime}\right\rangle\left\langle q|d+1, \ldots, n| p^{\prime}\right\rangle} .
\end{aligned}
$$

The first two contributions to (4.130) nearly cancel: the remainder reads

$$
\begin{aligned}
\mathcal{M}_{T}= & \frac{i}{2}(-g \sqrt{2})^{n+2} \sum_{\mathcal{P}(1 \ldots n)} \sum_{d=0}^{n}(\Omega[1, d])_{\ell}^{i}(\Omega[d+1, n])_{k}{ }^{j} \\
& \times \frac{\langle p q\rangle^{2} u^{\alpha}(q) p_{\alpha \dot{\alpha}} \bar{p}^{\dot{\alpha} \beta} u_{\beta}(q)}{\langle p q\rangle\left\langle q|1, \ldots, d| q^{\prime}\right\rangle\left\langle q|d+1, \ldots, n| p^{\prime}\right\rangle} \frac{1}{\left[p+p^{\prime}\right]^{2}} \\
+ & \frac{i}{2}(-g \sqrt{2})^{n+2} \sum_{\mathcal{P}(1 \ldots n)} \sum_{d=1}^{n}(\Omega[1, d])_{\ell}^{i}(\Omega[d+1, n])_{k}^{j} \\
& \times \frac{\left\langle 1 q^{\prime}\right\rangle}{\left\langle 1|q| q^{\prime}\right\rangle} \frac{\langle p q\rangle^{2} u^{\alpha}(q) p_{\alpha \dot{\alpha}} \bar{p}^{\dot{\alpha} \beta} u_{\beta}(q)}{\left\langle p|1, \ldots, d| q^{\prime}\right\rangle\left\langle q|d+1, \ldots, n| p^{\prime}\right\rangle} \frac{1}{\left[p+p^{\prime}\right]^{2}} .
\end{aligned}
$$

The two terms in (4.131) have very similar structure, although the sum in the first contribution contains an extra term. Taking this into account and pulling 
out common factors gives

$$
\begin{aligned}
\mathcal{M}_{T}=\frac{i}{2}( & -g \sqrt{2})^{n+2} \sum_{\mathcal{P}(1 \ldots n)} \delta^{i} \ell(\Omega[1, n])_{k}^{j} \\
& \times \frac{\langle p q\rangle^{2}\langle q p\rangle\left\langle p^{\prime} p\right\rangle^{*}\left\langle p^{\prime} q\right\rangle}{\langle p q\rangle\left\langle q q^{\prime}\right\rangle\left\langle q|1, \ldots, n| p^{\prime}\right\rangle} \frac{1}{\left\langle p^{\prime} p\right\rangle\left\langle p^{\prime} p\right\rangle^{*}} \\
+ & \frac{i}{2}(-g \sqrt{2})^{n+2} \sum_{\mathcal{P}(1 \ldots n)} \sum_{d=1}^{n}(\Omega[1, d])^{i} \ell(\Omega[d+1, n])_{k}^{j} \\
& \times \frac{\langle p q\rangle^{2}\langle q p\rangle\left\langle p^{\prime} p\right\rangle^{*}\left\langle p^{\prime} q\right\rangle}{\left\langle p|1, \ldots, d| q^{\prime}\right\rangle\left\langle q|d+1, \ldots, n| p^{\prime}\right\rangle} \frac{1}{\left\langle p^{\prime} p\right\rangle\left\langle p^{\prime} p\right\rangle^{*}} \\
& \times\left[\frac{\langle p 1\rangle}{\langle p|q| 1\rangle}+\frac{\left\langle 1 q^{\prime}\right\rangle}{\left\langle 1|q| q^{\prime}\right\rangle}\right]
\end{aligned}
$$

where we have used the relations in Appendix A to write everything in terms of spinor inner products. The square brackets in the second piece of (4.132) may be summed using (A.15). Doing this, plus a little more rearrangement produces

$$
\begin{aligned}
\mathcal{M}_{T}= & -\frac{i}{2}(-g \sqrt{2})^{n+2} \sum_{\mathcal{P}(1 \ldots n)} \frac{\delta^{i} \ell\left\langle p q^{\prime}\right\rangle}{\left\langle p q^{\prime}\right\rangle} \frac{(\Omega[1, n])_{k}{ }^{j}\left\langle q p^{\prime}\right\rangle}{\left\langle q|1, \ldots, n| p^{\prime}\right\rangle} \frac{\langle p q\rangle^{2}}{\left\langle p p^{\prime}\right\rangle\left\langle q q^{\prime}\right\rangle} \\
& -\frac{i}{2}(-g \sqrt{2})^{n+2} \sum_{\mathcal{P}(1 \ldots n)} \sum_{d=1}^{n} \frac{(\Omega[1, d])^{i} \ell\left\langle p q^{\prime}\right\rangle}{\left\langle p|1, \ldots, d| q^{\prime}\right\rangle} \frac{(\Omega[d+1, n])_{k}{ }^{j}\left\langle q p^{\prime}\right\rangle}{\left\langle q|d+1, \ldots, n| p^{\prime}\right\rangle} \frac{\langle p q\rangle^{2}}{\left\langle p p^{\prime}\right\rangle\left\langle q q^{\prime}\right\rangle} .
\end{aligned}
$$

Obviously, the first term of (4.133) simply extends the sum in the second piece to include $d=0$ once more. Combination of (4.133) with $\mathcal{M}_{5}$ (equation (4.29)) produces the final result:

$$
\begin{aligned}
& \mathcal{M}_{k \ell}^{i j}\left(p^{-}, q^{-} ; 1^{+}, \ldots, n^{+} ; p^{\prime+}, q^{\prime+}\right)= \\
&=-\frac{i}{2}(-g \sqrt{2})^{n+2} \sum_{\mathcal{P}(1 \ldots n)} \sum_{d=0}^{n}\left\{\frac{(\Omega[1, d])^{i}{ }_{\ell}\left\langle p q^{\prime}\right\rangle}{\left\langle p|1, \ldots, d| q^{\prime}\right\rangle} \frac{(\Omega[d+1, n])_{k}{ }^{j}\left\langle q p^{\prime}\right\rangle}{\left\langle q|d+1, \ldots, n| p^{\prime}\right\rangle}\right. \\
&\left.-\frac{1}{N} \frac{(\Omega[1, d])^{i j}\left\langle p p^{\prime}\right\rangle}{\left\langle p|1, \ldots, d| p^{\prime}\right\rangle} \frac{(\Omega[d+1, n])_{k \ell}\left\langle q q^{\prime}\right\rangle}{\left\langle q|d+1, \ldots, n| q^{\prime}\right\rangle}\right\} \frac{\langle p q\rangle^{2}}{\left\langle p p^{\prime}\right\rangle\left\langle q q^{\prime}\right\rangle} .
\end{aligned}
$$

The expression obtained here agrees with the result of Mangano from reference [6]. Note that (4.134) does not include the crossed-channel contribution which is required if both quark lines are the same species and helicity. This is easily written down from (4.134). 
As mentioned earlier, it is straightforward to generate the amplitudes for the remaining two quark helicity combinations. The results read

$$
\begin{aligned}
& \mathcal{M}_{k \ell}^{i j}\left(p^{+}, q^{-} ; 1^{+}, \ldots, n^{+} ; p^{\prime-}, q^{\prime+}\right)=\frac{\left\langle p^{\prime} q\right\rangle^{2}}{\langle p q\rangle^{2}} \mathcal{M}_{k \ell}^{i j}\left(p^{-}, q^{-} ; 1^{+}, \ldots, n^{+} ; p^{++}, q^{\prime+}\right), \\
& \mathcal{M}_{k \ell}^{i j}\left(p^{+}, q^{+} ; 1^{+}, \ldots, n^{+} ; p^{\prime-}, q^{\prime-}\right)=\frac{\left\langle p^{\prime} q^{\prime}\right\rangle^{2}}{\langle p q\rangle^{2}} \mathcal{M}_{k \ell}^{i j}\left(p^{-}, q^{-} ; 1^{+}, \ldots, n^{+} ; p^{\prime+}, q^{\prime+}\right) .
\end{aligned}
$$

Thus, we see that the only effect of changing the quark helicities is a minor change in one of the factors; the gross structure of the amplitude remains unaltered. These two amplitudes also agree with reference [6].

\section{CONCLUSION}

The complexity of computing scattering amplitudes in QCD rapidly increases with the number of particles involved. It is, therefore, important to develop efficient techniques for computing different types of amplitudes. As an attempt in this dierection, we have studied a gluon current with two off-shell gluons. This object appears in the process (1.1). This modified gluon current has

one off-shell gluon, and one "special" gluon. The "special" gluon is off mass shell $\left(k^{2} \neq 0\right)$ but has a transverse polarization vector $(k \cdot \epsilon=0)$. We have obtained an expression for the modified gluon current in the case of like helicity gluons. We have applied this current to the computation of the process (1.1). In spite of the intermediate expressions for this amplitude being quite complicated, the final result assumes a relatively simple form.

\section{Acknowledgements:}

We would like to thank Dr. Michelangelo Mangano and Dr. Stephen Parke for their useful discussions about the result in reference [6].

This work was supported in part by the National Science Foundation. 


\section{APPENDIX: MULTISPINOR CONVENTIONS}

Below we list the important results of application of Weyl-van der Waerden spinor calculus to gauge theories. Readers interested in the details should refer to references [1] and [7].

We use the Weyl basis

$$
\gamma^{\mu}=\left(\begin{array}{cc}
0 & \sigma^{\mu} \\
\bar{\sigma}^{\mu} & 0
\end{array}\right)
$$

for the Dirac matrices. In (A.1), $\sigma^{\mu}$ and $\bar{\sigma}^{\mu}$ refer to the convenient Lorentzcovariant grouping of the $2 \times 2$ Pauli matrices plus the unit matrix:

$$
\begin{gathered}
\sigma^{\mu} \equiv(1, \vec{\sigma}), \\
\bar{\sigma}^{\mu} \equiv(1,-\vec{\sigma}),
\end{gathered}
$$

and satisfy the anticommutators

$$
\begin{aligned}
& \left(\bar{\sigma}^{\mu}\right)^{\dot{\alpha} \beta}\left(\sigma^{\nu}\right)_{\beta \dot{\beta}}+\left(\bar{\sigma}^{\nu}\right)^{\dot{\alpha} \beta}\left(\sigma^{\mu}\right)_{\beta \dot{\beta}}=2 g^{\mu \nu} \delta_{\dot{\beta}}^{\dot{\alpha}}, \\
& \left(\sigma^{\mu}\right)_{\alpha \dot{\beta}}\left(\bar{\sigma}^{\nu}\right)^{\dot{\beta} \beta}+\left(\sigma^{\nu}\right)_{\alpha \dot{\beta}}\left(\bar{\sigma}^{\mu}\right)^{\dot{\beta} \beta}=2 g^{\mu \nu} \delta_{\alpha}^{\beta} .
\end{aligned}
$$

To each Lorentz 4-vector there corresponds a rank two multispinor, formed from the contraction of the 4 -vector with $\sigma^{\mu}$ :

$$
\begin{aligned}
& \mathcal{J}_{\alpha \dot{\beta}}=\frac{1}{\sqrt{2}} \sigma_{\alpha \dot{\beta}}^{\mu} J_{\mu}, \\
& \overline{\mathcal{J}}^{\dot{\alpha} \beta}=\frac{1}{\sqrt{2}} \bar{\sigma}_{\mu}^{\dot{\alpha} \beta} J^{\mu} .
\end{aligned}
$$

For the purposes of normalization, it is convenient to use a different convention when converting momenta:

$$
k_{\alpha \dot{\beta}}=\sigma_{\alpha \dot{\beta}}^{\mu} k_{\mu},
$$




$$
\bar{k}^{\dot{\alpha} \beta}=\bar{\sigma}_{\mu}^{\dot{\alpha} \beta} k^{\mu}
$$

Useful consequences of (A.5) and (A.3) are

$$
\begin{aligned}
& \bar{k}^{\dot{\alpha} \beta} k_{\beta \dot{\beta}}=k^{2} \delta_{\dot{\beta}}^{\dot{\alpha}}, \\
& k_{\alpha \dot{\beta}} \bar{k}^{\dot{\beta} \beta}=k^{2} \delta_{\alpha}^{\beta} .
\end{aligned}
$$

The spinor indices may be raised and lowered using the 2-component antisymmetric tensor:

$$
\begin{gathered}
u^{\alpha}=\varepsilon^{\alpha \beta} u_{\beta}, \\
\bar{v}^{\dot{\alpha}}=\varepsilon^{\dot{\alpha} \dot{\beta}} \bar{v}_{\dot{\beta}}, \\
\varepsilon^{\alpha \beta}=\varepsilon_{\alpha \beta}, \\
\varepsilon^{\dot{\alpha} \dot{\beta}}=\varepsilon_{\dot{\alpha} \dot{\beta}}, \\
\varepsilon_{12}=\varepsilon_{\dot{1} \dot{2}}=1 .
\end{gathered}
$$

Many useful relations may be easily proven from the Schouten identity

$$
\delta_{\gamma}^{\alpha} \delta_{\delta}^{\beta}-\delta_{\delta}^{\alpha} \delta_{\gamma}^{\beta}+\varepsilon^{\alpha \beta} \varepsilon_{\gamma \delta}=0
$$

the generator of 2-component Fierz transformations.

We denote by $u(k)$ and $\bar{u}(k)$ the solutions to the 2 -component Weyl equations:

$$
\begin{aligned}
& \bar{k}^{\dot{\alpha} \beta} u_{\beta}(k)=0, \\
& \bar{u}_{\dot{\beta}}(k) \bar{k}^{\dot{\beta} \alpha}=0 .
\end{aligned}
$$

These two spinors are related by complex conjugation

$$
\bar{u}_{\dot{\alpha}}(k)=\left[u_{\alpha}(k)\right]^{*}
$$


and have the normalization

$$
u_{\alpha}(k) \bar{u}_{\dot{\alpha}}(k)=k_{\alpha \dot{\alpha}}
$$

It is useful to define a scalar product

$$
\langle 12\rangle \equiv u^{\alpha}\left(k_{1}\right) u_{\alpha}\left(k_{2}\right)
$$

which has two elementary properties

$$
\begin{gathered}
\langle 12\rangle=-\langle 21\rangle, \\
\langle 12\rangle\langle 12\rangle^{*}=2 k_{1} \cdot k_{2} .
\end{gathered}
$$

Contraction of $u_{\alpha}\left(k_{1}\right) u_{\beta}\left(k_{2}\right) u^{\gamma}\left(k_{3}\right) u^{\delta}\left(k_{4}\right)$ into (A.8) produces the extremely useful relation

$$
\langle 12\rangle\langle 34\rangle+\langle 13\rangle\langle 42\rangle+\langle 14\rangle\langle 23\rangle=0 .
$$

A second relation of great utility may be derived from (A.14):

$$
\frac{\langle 12\rangle}{\langle 1 q\rangle\langle q 2\rangle}+\frac{\langle 23\rangle}{\langle 2 q\rangle\langle q 3\rangle}=\frac{\langle 13\rangle}{\langle 1 q\rangle\langle q 3\rangle} .
$$

Equation (A.15) may be used to demonstrate that

$$
\sum_{j=\ell}^{m-1} \frac{\langle j j+1\rangle}{\langle j q\rangle\langle q j+1\rangle}=\frac{\langle\ell m\rangle}{\langle\ell q\rangle\langle q m\rangle} .
$$

A recurring structure is

$$
\langle p|1,2, \ldots, n| q\rangle \equiv\langle p 1\rangle\langle 12\rangle \cdots\langle n q\rangle
$$

We note the following basic properties of $\langle p|1,2, \ldots, n| q\rangle$ :

$$
\begin{gathered}
\langle p|| q\rangle \equiv\langle p q\rangle \\
\langle p|1,2, \ldots, j-1| j\rangle\langle j|j+1, j+2, \ldots, n| q\rangle=\langle p|1,2, \ldots, n| q\rangle
\end{gathered}
$$




$$
\langle q|n, n-1, \ldots, 1| p\rangle=(-1)^{n-1}\langle p|1,2, \ldots, n| q\rangle .
$$

Helicities \pm 1 for massless vector bosons may be described by

$$
\begin{aligned}
\epsilon_{\alpha \dot{\alpha}}\left(k^{+}\right) & \equiv \frac{u_{\alpha}(q) \bar{u}_{\dot{\alpha}}(k)}{\langle k q\rangle}, \\
\epsilon_{\alpha \dot{\alpha}}\left(k^{-}\right) & \equiv \frac{u_{\alpha}(k) \bar{u}_{\dot{\alpha}}(q)}{\langle k q\rangle^{*}},
\end{aligned}
$$

where $q$ is any null-vector such that $k \cdot q \neq 0$. As the choice of $q$ does not affect any physics result, we will refer to $u(q)$ and $\bar{u}(q)$ as gauge spinors. The corresponding polarization vectors $\epsilon^{\mu}(k)$ defined through (A.4) differ from the "standard" polarization vectors

$$
\begin{gathered}
\varepsilon_{0}^{\mu}\left(k^{ \pm}\right)=\left(0, \mp \frac{1}{\sqrt{2}}, \frac{-i}{\sqrt{2}}, 0\right), \\
k^{\mu}=(k, 0,0, k),
\end{gathered}
$$

by a phase and a gauge transformation depending on $q \cdot[1]$

To save accounting for a large number of indices, an efficient method is to initially write quantities in the usual formalism and then convert to multispinor notation at a later stage using the substitutions

$$
\begin{gathered}
k \cdot k^{\prime}=\frac{1}{2} \bar{k}^{\dot{\alpha} \alpha} k_{\alpha \dot{\alpha}}^{\prime}=\frac{1}{2} k_{\alpha \dot{\alpha}} \bar{k}^{\prime \dot{\alpha} \alpha}, \\
k \cdot \epsilon\left(k^{\prime}\right)=\frac{1}{\sqrt{2}} \bar{k}^{\dot{\alpha} \alpha} \epsilon_{\alpha \dot{\alpha}}\left(k^{\prime}\right)=\frac{1}{\sqrt{2}} k_{\alpha \dot{\alpha}} \bar{\epsilon}^{\dot{\alpha} \alpha}\left(k^{\prime}\right), \\
\epsilon(k) \cdot \epsilon\left(k^{\prime}\right)=\bar{\epsilon}^{\dot{\alpha} \alpha}(k) \epsilon_{\alpha \dot{\alpha}}\left(k^{\prime}\right)=\epsilon_{\alpha \dot{\alpha}}(k) \bar{\epsilon}^{\dot{\alpha} \alpha}\left(k^{\prime}\right),
\end{gathered}
$$

for Lorentz dot products and

$$
\begin{gathered}
\frac{1}{2}\left(1-\gamma_{5}\right) \psi \longrightarrow \psi_{\alpha}, \\
\frac{1}{2}\left(1+\gamma_{5}\right) \psi \longrightarrow \psi^{\dot{\alpha}}, \\
\frac{1}{2}\left(1-\gamma_{5}\right), J_{2} \frac{1}{2}\left(1+\gamma_{5}\right) \longrightarrow \sqrt{2} \mathcal{J}_{\alpha \dot{\alpha}},
\end{gathered}
$$




$$
\begin{gathered}
\frac{1}{2}\left(1+\gamma_{5}\right), J \frac{1}{2}\left(1-\gamma_{5}\right) \longrightarrow \sqrt{2} \overline{\mathcal{J}}^{\dot{\alpha} \alpha}, \\
\frac{1}{2}\left(1-\gamma_{5}\right) \not k \frac{1}{2}\left(1+\gamma_{5}\right) \longrightarrow k_{\alpha \dot{\alpha}}, \\
\frac{1}{2}\left(1+\gamma_{5}\right) \not k \frac{1}{2}\left(1-\gamma_{5}\right) \longrightarrow \bar{k}^{\dot{\alpha} \alpha},
\end{gathered}
$$

in strings of Dirac matrices. Note the unequal treatments of momenta versus other 4-vectors caused by the conventions (A.4) and (A.5). 


\section{REFERENCES}

1. F. A. Berends and W. T. Giele, Nucl. Phys. B306 (1988) 759; C. Dunn and T.-M. Yan, Nucl. Phys. B352 (1991) 402; C. Dunn, thesis, Cornell University $(1990)$

2. J. Schwinger, Particles, Sources and Fields, Vol. I, Addison Wesley, 1970; Ann. Phys. 119 (1979) 192

3. The spinor technique was first introduced by the CALCUL collaboration, in the context of massless Abelian gauge theory: P. De Causmaecker, R. Gastmans, W. Troost, and T.T. Wu, Phys. Lett. 105B (1981) 215; P. De Causmaecker, R. Gastmans, W. Troost and T.T. Wu, Nucl. Phys. B206 (1982) 53; F. A. Berends, R. Kleiss, P. De Causmaecker, R. Gastmans, W. Troost and T.T. Wu, Nucl. Phys. B206 (1982) 61; F.A. Berends, P. De Causmaecker, R. Gastmans, R. Kleiss, W. Troost and T.T. Wu, Nucl. Phys. B239 (1984) 382; B239 (1984) 395; B264 (1986) 243; B264 (1986) 265

By now, many papers have been published on the subject. A partial list of references follows.

P. De Causmaecker, thesis, Leuven University (1983); R. Farrar and F. Neri, Phys. Lett. 130B (1983) 109; R. Kleiss, Nucl. Phys. B241 (1984) 61; Z. Xu, D.H. Zhang and Z. Chang, Tsingua University preprint TUTP-84/3, 84/4, and 84/5a (1984), and Nucl. Phys. B291 (1987) 392; J.F. Gunion and Z. Kunszt, Phys. Lett. 161B (1985) 333; F.A. Berends, P.H. Davereldt and R. Kleiss, Nucl. Phys. B253 (1985) 441; R. Kleiss and W.J. Stirling, Nucl. Phys. B262 (1985) 235; J.F. Gunion and Z. Kunszt, Phys. Lett. 159B (1985) 167; 161B (1985) 333; S.J. Parke and T.R. Taylor, Phys. Rev. Lett. 56 (1986) 2459; Z. Kunszt, Nucl. Phys. B271 (1986) 333; J.F. Gunion and J. Kalinowski, Phys. Rev. D34 (1986) 2119; R. Kleiss and W.J. Stirling, Phys. Lett. 179B (1986) 159; M. Mangano and S.J. Parke, Nucl. Phys. B299 (1988) 673; M. Mangano, S.J. Parke and Z. Xu, Nucl. Phys. B298 (1988) 653; D.A. Kosower, B.-H. Lee and V.P. Nair, Phys. Lett. 201B (1988) 85; M. Mangano and S.J. Parke, Nucl. Phys. B299 (1988) 673; F.A. Berends and W.T. Giele, Nucl. Phys. B313 (1989) 595; M. Mangano, Nucl. Phys. B315 (1989) 391; D.A. Kosower, Nucl. Phys. B335 (1990) 23; D.A. Kosower, Phys. Lett. B254 (1991) 439; Z. Bern and D.A. Kosower, Nucl. Phys. B379 (1992) 451; C.S. Lam, McGill preprint McGill/92-32 (1992)

4. Many of the results for processes containing six or fewer particles are collected in R. Gastmans and T.T. Wu, The Ubiquitous Photon: Helicity Method for QED and QCD (Oxford University Press, New York, 1990)

5. M. Mangano and S. Parke, Phys. Reports 200 (1991) 301 
6. M. Mangano, Nucl. Phys. B309 (1988) 461

7. For a brief introduction to properties of two-component Weyl-van der Waerden spinors, see, for example, M. F. Sohnius, Phys. Reports 128 (1985) 39 